\title{
The ChemCam Instrument Suite on the Mars Science Laboratory (MSL) Rover: Body Unit and Combined System Tests
}

Roger C. Wiens • Sylvestre Maurice • Bruce Barraclough • Muriel Saccoccio • Walter C. Barkley · James F. Bell III • Steve Bender · John Bernardin • Diana Blaney • Jennifer Blank · Marc Bouyé • Nathan Bridges • Nathan Bultman · Phillippe Caïs · Robert C. Clanton • Benton Clark • Samuel Clegg • Agnes Cousin • David Cremers • Alain Cros • Lauren DeFlores • Dorothea Delapp · Robert Dingler • Claude D'Uston • M. Darby Dyar · Tom Elliott • Don Enemark • Cecile Fabre • Mike Flores • Olivier Forni · Olivier Gasnault • Thomas Hale · Charles Hays • Ken Herkenhoff • Ed Kan • Laurel Kirkland • Driss Kouach • David Landis • Yves Langevin • Nina Lanza · Frank LaRocca · Jeremie Lasue · Joseph Latino • Daniel Limonadi • Chris Lindensmith • Cynthia Little • Nicolas Mangold • Gerard Manhes • Patrick Mauchien • Christopher McKay • Ed Miller • Joe Mooney • Richard V. Morris • Leland Morrison - Tony Nelson • Horton Newsom • Ann Ollila • Melanie Ott · Laurent Pares · René Perez • Franck Poitrasson • Cheryl Provost • Joseph W. Reiter • Tom Roberts • Frank Romero • Violaine Sautter • Steven Salazar • John J. Simmonds • Ralph Stiglich • Steven Storms • Nicolas Striebig • Jean-Jacques Thocaven · Tanner Trujillo • Mike Ulibarri • David Vaniman • Noah Warner · Rob Waterbury · Robert Whitaker · James Witt • Belinda Wong-Swanson

Received: 12 November 2011 / Accepted: 22 May 2012 / Published online: 21 June 2012

(C) The Author(s) 2012. This article is published with open access at Springerlink.com

R.C. Wiens $(\bowtie) \cdot$ B. Barraclough $\cdot$ W.C. Barkley $\cdot$ S. Bender $\cdot$ J. Bernardin $\cdot$ N. Bultman R.C. Clanton - S. Clegg - D. Delapp · R. Dingler - D. Enemark · M. Flores · T. Hale · N. Lanza · J. Lasue - J. Latino - C. Little · L. Morrison - T. Nelson · F. Romero · S. Salazar · R. Stiglich •

S. Storms · T. Trujillo $\cdot$ M. Ulibarri $\cdot$ D. Vaniman $\cdot$ R. Whitaker $\cdot$ J. Witt Los Alamos National Laboratory, Los Alamos, NM 87545, USA e-mail: rwiens@lanl.gov

S. Maurice · M. Bouyé · A. Cousin · A. Cros · C. D’Uston · O. Forni · O. Gasnault · D. Kouach · J. Lasue · L. Pares · F. Poitrasson · N. Striebig · J.-J. Thocaven Institut de Recherche en Astrophysique et Planetologie (IRAP), Université Paul Sabatier, UPS-OMP, 31400 Toulouse, France

S. Maurice · M. Bouyé · A. Cousin · A. Cros · C. D’Uston · O. Forni · O. Gasnault · D. Kouach ·

J. Lasue · L. Pares · F. Poitrasson · N. Striebig · J.-J. Thocaven

CNRS, IRAP, 9 Av. Colonel Roche, BP 44346, 31028 Toulouse cedex 4, France 
M. Saccoccio $\cdot$ R. Perez

Centre National d'Etudes Spatiales, Toulouse, France

J.F. Bell III · C. Hays

School of Earth and Space Exploration, Arizona State University, Tempe, AZ 85287-1404, USA

D. Blaney · L. DeFlores · T. Elliott · E. Kan · D. Limonadi · C. Lindensmith · E. Miller · J.W. Reiter ·

T. Roberts · J.J. Simmonds · N. Warner

Jet Propulsion Laboratory, California Institute of Technology, Pasadena, CA, USA

J. Blank

Bay Area Environmental Research Institute, Sonoma, CA, USA

N. Bridges

Applied Physics Laboratory, Johns Hopkins University, Laurel, MD, USA

P. Caïs

Laboratoire d'Astrophysique de Bordeaux, Université of Bordeaux, 33299, Bordeaux, France

B. Clark

Space Science Institute, Boulder, CO, USA

D. Cremers

Applied Research Associates, Albuquerque, NM, USA

M. Darby Dyar

Dept. of Astronomy, Mount Holyoke College, South Hadley, MA, USA

C. Fabre

Géologie et Gestion des Ressources Minérales et Energétiques, Université de Nancy, 54506 Vandoeuvre lès Nancy, France

K. Herkenhoff

U.S. Geological Survey, Astrogeology Science Center, Flagstaff, AZ 86001-1698, USA

L. Kirkland

Lunar and Planetary Institute, Houston, TX, USA

D. Landis

The Charles Stark Draper Laboratory, Tampa, FL, USA

Y. Langevin

Institut d'Astrophysique Spatiale, Université de Paris-Sud, 91405 Orsay, France

N. Lanza $\cdot$ H. Newsom · A. Ollila

University of New Mexico, Albuquerque, NM, USA

F. LaRocca $\cdot$ M. Ott

Goddard Space Flight Center, Greenbelt, MD, USA

N. Mangold

LPGN, CNRS, UMR6112, Université Nantes, Nantes, France

G. Manhes

Institute de Physique du Globe de Paris, 75252, Paris, France

P. Mauchien

Commissariat à l'Energie Atomique et aux Énergies Alternatives, 91191 Gif Sur Yvette, France 


\begin{abstract}
The ChemCam instrument suite on the Mars Science Laboratory (MSL) rover Curiosity provides remote compositional information using the first laser-induced breakdown spectrometer (LIBS) on a planetary mission, and provides sample texture and morphology data using a remote micro-imager (RMI). Overall, ChemCam supports MSL with five capabilities: remote classification of rock and soil characteristics; quantitative elemental compositions including light elements like hydrogen and some elements to which LIBS is uniquely sensitive (e.g., $\mathrm{Li}, \mathrm{Be}, \mathrm{Rb}, \mathrm{Sr}, \mathrm{Ba}$ ); remote removal of surface dust and depth profiling through surface coatings; context imaging; and passive spectroscopy over the 240$905 \mathrm{~nm}$ range. ChemCam is built in two sections: The mast unit, consisting of a laser, telescope, RMI, and associated electronics, resides on the rover's mast, and is described in a companion paper. ChemCam's body unit, which is mounted in the body of the rover, comprises an optical demultiplexer, three spectrometers, detectors, their coolers, and associated electronics and data handling logic. Additional instrument components include a $6 \mathrm{~m}$ optical fiber which transfers the LIBS light from the telescope to the body unit, and a set of onboard calibration targets. ChemCam was integrated and tested at Los Alamos National Laboratory where it also underwent LIBS calibration with 69 geological standards prior to integration with the rover. Post-integration testing used coordinated mast and instrument commands, including LIBS line scans on rock targets during system-level thermal-vacuum tests. In this paper we describe the body unit, optical fiber, and calibration targets, and the assembly, testing, and verification of the instrument prior to launch.
\end{abstract}

Keywords Mars · Laser induced breakdown spectroscopy · LIBS · RMI · Mars Science Laboratory $\cdot$ MSL $\cdot$ Curiosity $\cdot$ Gale Crater $\cdot$ Laser plasma $\cdot$ ChemCam

\title{
Abbreviations \\ 2D Two dimensional \\ ASD Analytical Spectral Devices \\ ATLO Assembly, test, and launch operations phase \\ BIF Banded iron formation, a Precambrian layered sediment
}

\author{
J. Blank · C. McKay \\ NASA Ames Research Center, Mountain View, CA, USA \\ J. Mooney · C. Provost \\ CeramOptec Industries, Inc., East Longmeadow, MA, USA
}

R.V. Morris

NASA Johnson Space Center, Houston, TX, USA

\section{Sautter}

Laboratoire de Minéralogie et Cosmochimie du Muséum, Muséum National d'Histoire Naturelle, 75005 Paris, France

R. Waterbury

Ocean Optics, Inc., Dunedin, FL, USA

B. Wong-Swanson

Innov8 LLC, Santa Fe, NM, USA

B. Barraclough - S. Bender · D. Vaniman

Planetary Science Institute, Tucson, AZ, USA 


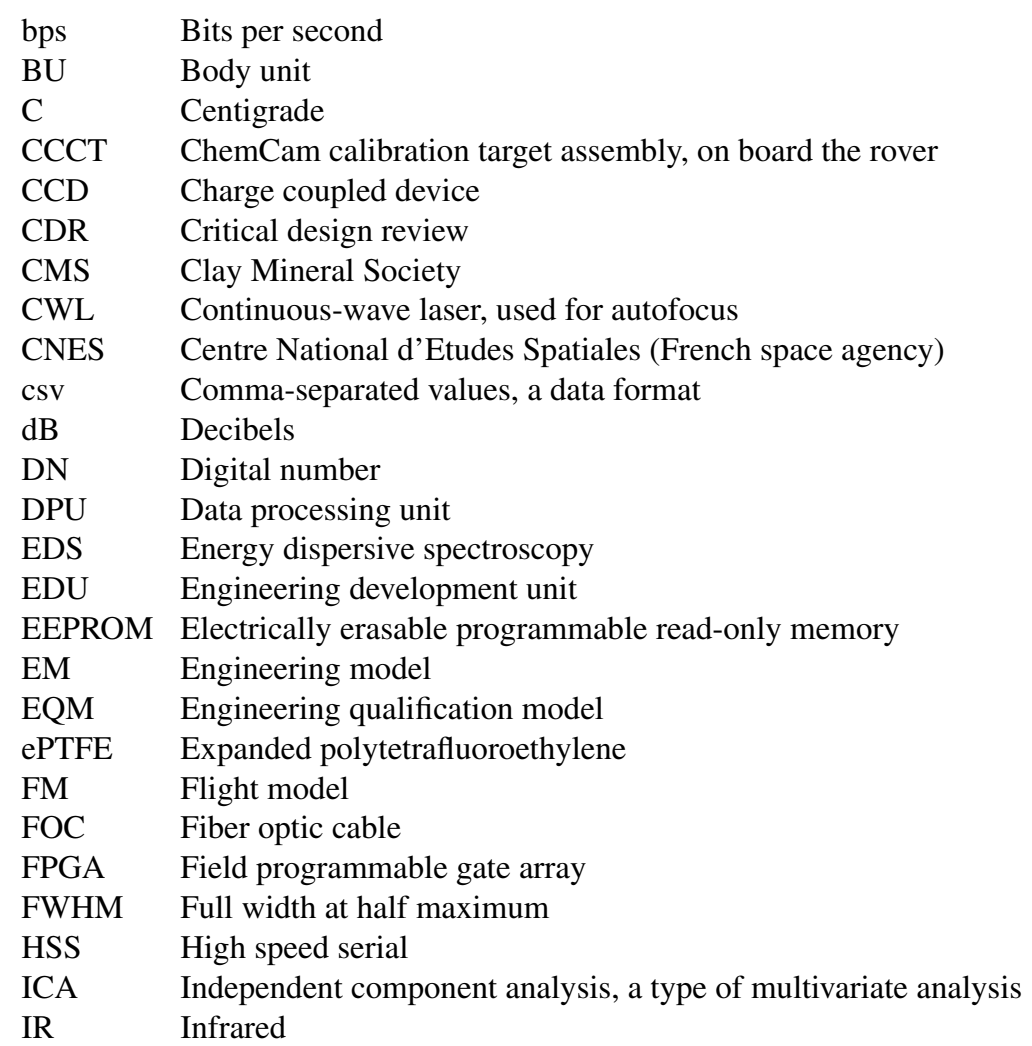

IRAP Institut de Recherche en Astrophysique et Planetologie, the lead ChemCam institution in France

JPL Jet Propulsion Laboratory

$\mathrm{kB} \quad$ Kilo bytes

kbps Kilo bits per second

$\mathrm{kHz} \quad$ Kilo Hertz

LANL Los Alamos National Laboratory

LIBS Laser-induced breakdown spectroscopy

LVDS Low voltage differential signal

LVPS Low voltage power supply

LPM Lines per millimeter

Mbps Million bits per second

MER Mars Exploration Rovers, launched 2003

$\mathrm{MHz} \quad$ Million Hertz (per second)

MSLICE Mars Science Laboratory interface

MU Mast unit

mV Milli Volts

MVA Multivariate analysis

NA Numerical aperture

ng Nanograms

$\mathrm{OH} \quad$ Oxygen and hydrogen

OOI Ocean Optics, Incorporated

PDR Preliminary design review 


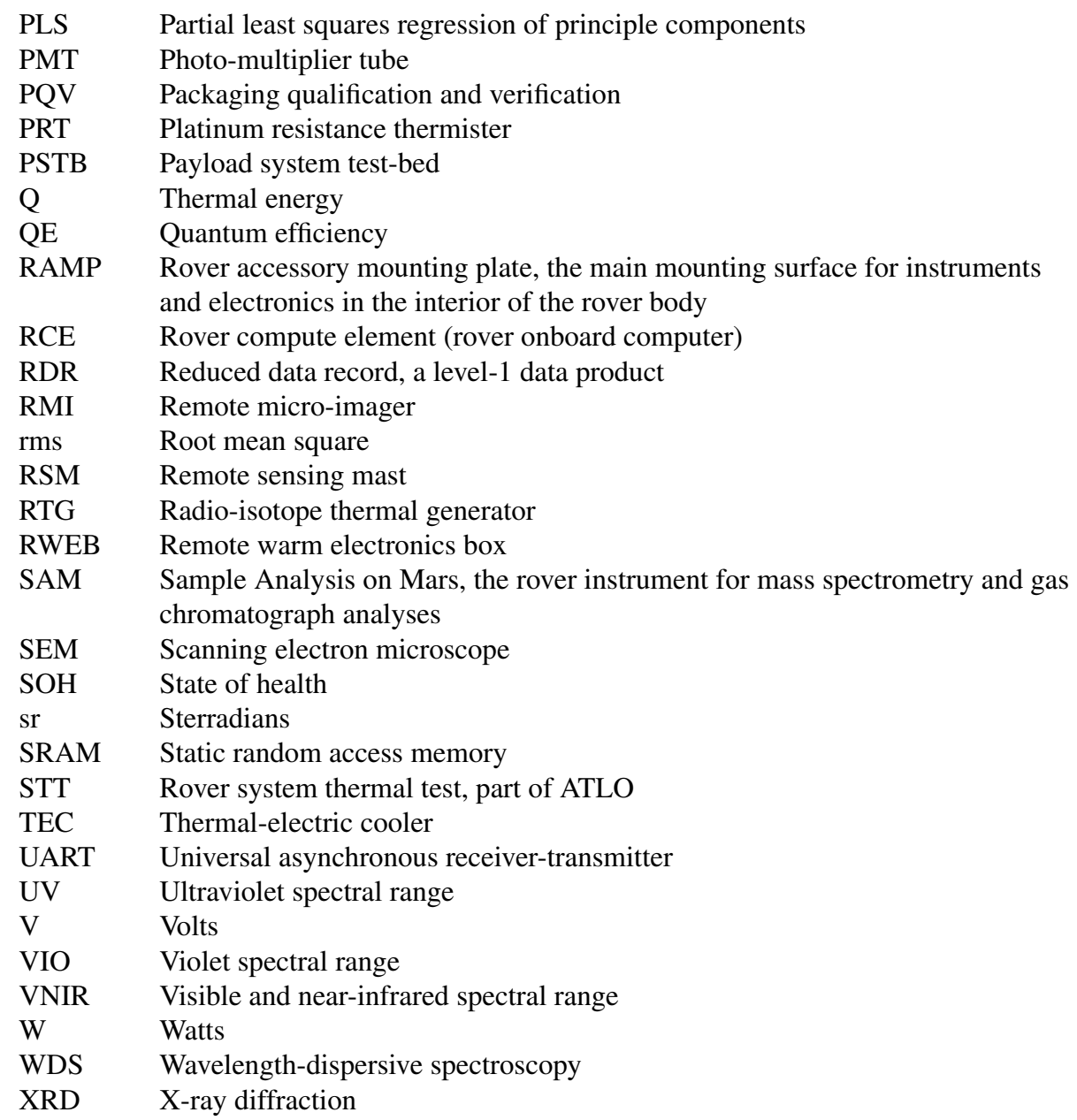

\section{Introduction}

The NASA Mars Exploration Program has seen an evolution in instrumentation for determining the composition of rock and soil samples. Landing in 1997, the Sojourner rover carried an alpha-proton X-ray spectrometer (APXS) for chemical analysis of rocks and soils (e.g., Brückner et al. 2003). However, the rover lacked a means to brush away surface dust and to grind through alteration rinds to analyze unweathered surfaces. The Mars Exploration Rovers' (MER) payload included a Mössbauer contact spectrometer along with an APXS instrument, and added a Rock Abrasion Tool (RAT) (Squyres et al. 2003) to remove surface dust and penetrate to rock interiors by grinding. MER also flew a multispectral panoramic camera (Pancam; Bell et al. 2003) and a Mini Thermal Emission Spectrometer (Mini-TES; Christensen et al. 2003) for remote sensing mineralogical analyses. MER could thus perform remote mineralogical analyses and analyze clean rock surfaces upon contact, but it could not analyze cleaned rock surfaces remotely.

In 2004, shortly after the MER landing, the ChemCam team proposed an active remote sensing experiment for the Mars Science Laboratory (MSL) rover. ChemCam combines 
Fig. 1 A ball of plasma erupts on the surface of a $3 \mathrm{~cm}$ diameter iron pyrite crystal in the Mars calibration chamber approximately $3 \mathrm{~m}$ from the ChemCam instrument. The plasma in this image was produced in air; plasmas under Mars pressure are brighter and larger

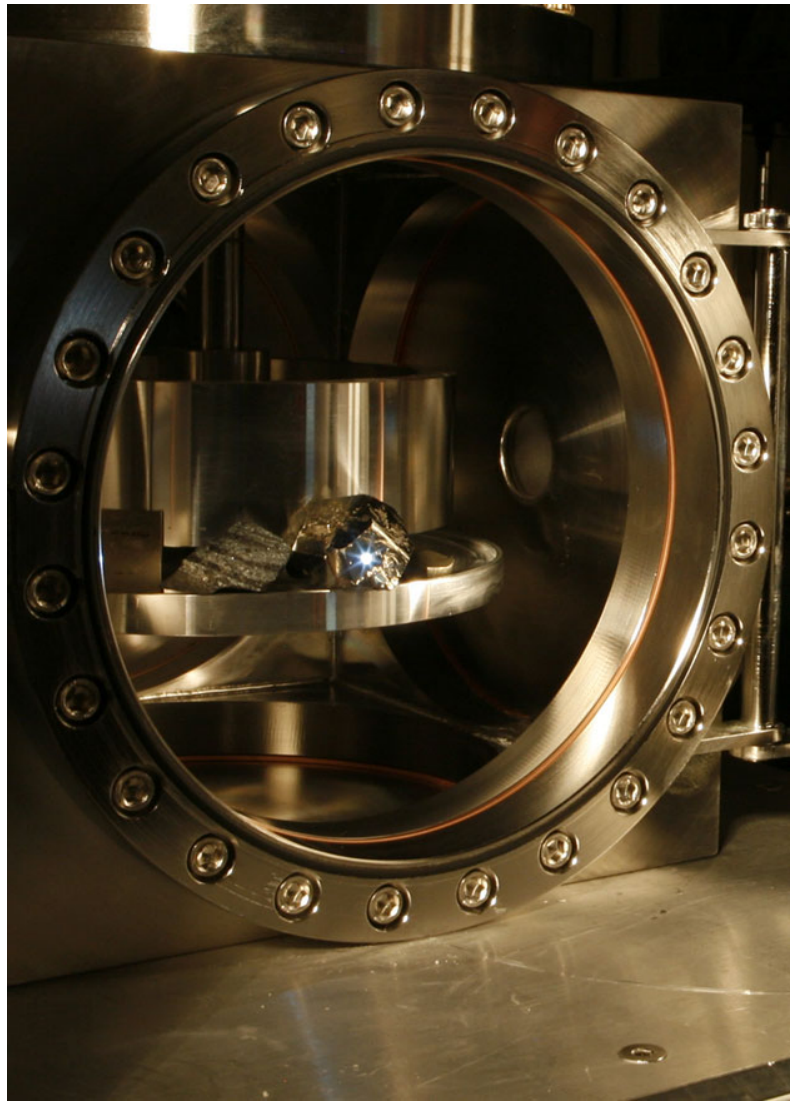

a laser-induced breakdown spectroscopy (LIBS) technique to provide elemental compositions, and a Remote Micro-Imager (RMI) for context imaging of the small $(350-550 \mu \mathrm{m})$ LIBS observation points. ChemCam is the first flight instrument with the capability to remotely clean and obtain depth profiles of samples by laser ablation.

In the LIBS technique, brief but powerful laser pulses are focused on a target. The high photon flux results in ablation of atoms and atomic clusters in electronically excited states, typically with a temperature of $\sim 10^{4} \mathrm{~K}$. As the ions and atoms decay to lower energy levels, photons are emitted at wavelengths that are unique to each element. An image of a LIBS plasma produced by ChemCam is shown in Fig. 1. The ChemCam laser beam is invisible $(\lambda=1067 \mathrm{~nm})$, so all of the light seen in the image is from the plasma, which lasts up to $\sim 20 \mu \mathrm{s}$. The LIBS spectrum covers a range from the deep ultraviolet to the infrared (Kaski et al. 2003; Radziemski et al. 2007), however, characteristic emission lines exist for essentially all of the elements within a range from 240 to $850 \mathrm{~nm}$ (i.e., the near ultraviolet to the near infrared). In remote sensing applications, in which the sample is located at a distance from the LIBS instrument, the light generated by the laser-induced plasma is collected by a telescope and transmitted, in most cases via optical fiber, to spectrometers that spectrally disperse the light onto detectors such as charge coupled devices (CCDs), which convert the signal into charge for electronic detection and quantification (cf. Sallé et al. 2007).

LIBS has been under development almost since the advent of the laser (cf. Cremers and Radziemski 2006). LIBS was first used at remote or stand-off distances in the late 1980s 
(Cremers 1987). Portable LIBS instruments became a reality in the late 1990s (e.g., Wiens et al. 2002) with the development of compact lasers and spectrometers. Observation distances for remote LIBS have increased to $>150 \mathrm{~m}$ in some experiments (Rohwetter et al. 2005). Combining LIBS and Raman spectroscopy into a single instrument has also been undertaken successfully (e.g., Wiens et al. 2005; Sharma et al. 2007).

LIBS analytical results are complicated by chemical matrix effects in which the presence of one element may affect the relationship between photon intensity and inferred abundance of another element (e.g., Dudragne et al. 1998). Multivariate analysis (MVA) techniques now allow correction for chemical matrix effects when a proper training set of standards is used (e.g., Sirven et al. 2006; Clegg et al. 2009; Forni et al. 2009; Tucker et al. 2010; Dyar et al. 2011; Anderson et al. 2011). Application of MVA has significantly improved the speed, accuracy, and precision of major element analyses of sample sets with a large range of variables, such as geological samples. Further treatment of MVA in the context of ChemCam calibration will be given in a future paper.

The LIBS technique is unique in many ways relative to previous planetary compositional analysis techniques:

- LIBS provides remote analyses without the need for sample preparation.

- Multiple laser pulses can remove dust and weathering rinds from remote samples. ChemCam's ability to depth profile into samples is described later in this paper.

- Simultaneous analytical capability extends to nearly all elements except the noble gases. This capability includes abundance determinations of the light elements $\mathrm{H}, \mathrm{Li}, \mathrm{Be}, \mathrm{B}$, $\mathrm{C}, \mathrm{N}$, and $\mathrm{O}$, which cannot typically be analyzed by X-ray techniques. Many of these elements are critical to understanding organic chemistry and to habitability issues.

- LIBS has very low detection limits for certain elements, specifically the alkalis and alkaline earths. These detection limits can be in the parts per million range even for relatively simple remote LIBS experiments.

- LIBS analyses are rapid, in essence consisting of a single laser pulse and the collection of a spectrum from the resulting plasma. LIBS spectra are usually averaged or summed together to improve counting statistics. For remote LIBS the observation time includes the interval it takes to re-focus the telescope at different locations. Overall, observation of one point on the sample takes approximately six minutes with ChemCam. (Downlink of the data from the rover to Earth usually occurs some hours later.)

- All LIBS observations must be done on a small footprint dictated by the power density required to achieve plasma. LIBS observation footprints are always less than one millimeter in diameter. This high spatial resolution is very useful for identifying small features. For representative whole-rock compositions of heterogeneous samples a mean of multiple observation points must be taken.

Given the small LIBS observation footprints, it is imperative to understand and document the contexts of the measurements. The accompanying RMI instrument provides these contexts and allows, for example, laboratory replication of target surfaces in order to quantify the influences of surface morphology (e.g., sample heterogeneity, coatings, weathering rinds) influences on the LIBS spectra.

ChemCam's development involved both NASA and the Centre National d'Etudes Spatiales (CNES), which is the French space agency. The instrument was built in two major sections: (1) a mast unit (MU) to send the laser beam and receive the LIBS signals and images, and (2) a body unit (BU) to collect and process the LIBS signals, control the instrument, and interface with the rover. The MU was developed by academic laboratories and CNES, under the leadership of the Institut de Recherche en Astrophysique et Planétologie 
(IRAP, formerly CESR). Los Alamos National Laboratory (LANL) led development of the $\mathrm{BU}$, funded by NASA. A description of the MU is given in a companion paper (Maurice et al. 2012). This paper describes the BU and two other components: an assembly of onboard calibration targets and a mast-to-body optical fiber, and reports on the testing and performance of the integrated instrument suite.

ChemCam equips the MSL rover with five overall capabilities: (1) sample classification, (2) quantitative analyses, (3) remote depth profiling, (4) context images, and (5) passive spectroscopy. The primary purpose of a remote sensing instrument on a rover such as $\mathrm{Cu}-$ riosity is to rapidly classify samples in the vicinity of the rover so that the rover's operating team can identify the samples of greatest interest on which to use the rover's other instruments. Acquisition of samples for use in the in-situ instruments can take up to several sols (Mars days), and analyses can take several more sols. An observation with ChemCam takes approximately six minutes once the instrument is ready, which is orders of magnitude faster than training in-situ instruments on each and every potential sample on Mars. Methods of sample classification using ChemCam LIBS are described briefly in Sect. 7.2.

ChemCam uses MVA data processing methods, computed on downlinked data, to obtain quantitative elemental compositions. While other instruments on MSL can obtain more accurate compositions on most elements which the rover contacts, the quantitative compositions obtained by ChemCam will provide unique data on a much larger number of analyses than obtained with the other instruments. ChemCam will provide exclusive information on elements of which LIBS is uniquely capable, including the light elements and those mentioned above for which LIBS has the best sensitivity. Unique quantitative compositional information extends to contexts exclusively sampled by ChemCam: samples that are inaccessible to the rover's arm and fine-scale sampling provided by the laser beam. The latter allows depth profiling at a fine scale, permitting the study of surface coatings and weathering rinds on rocks. Quantitative elemental compositions from other rover instruments in this context will be difficult due to the much larger size of the probes (sensor head in the case of APXS, drill tip size to provide samples for other instruments).

The final two capabilities are context imaging and passive spectroscopy. Given the small scale (350-550 $\mu \mathrm{m}$ diameter) of the LIBS observation, routine context images provided within the same experiment, with resolution down to $200 \mu \mathrm{m}$, were considered imperative; hence the inclusion of the RMI imager in the ChemCam package. MAHLI, with its imaging resolution to $\sim 20 \mu \mathrm{m}$, will also aid in providing additional context observations to LIBS observation pits. The final capability to be mentioned, that of passive spectroscopy, comes as a by-product of the instrumentation needed for LIBS, at least over the same spectral range. While largely undeveloped, this capability is likely to be utilized once Curiosity is on the surface of Mars.

\section{Instrument Overview, Requirements, and Development History}

This section gives an overview of the instrument, describes the instrument requirements, and provides an overview of the ChemCam development history.

\subsection{Instrument Overview}

An overview diagram of ChemCam is shown in Fig. 2. The instrument consists of two major sections, the MU, housing the laser, RMI, telescope, and associated electronics, and the BU, consisting of an optical demultiplexer, three spectrometers covering different spectral ranges, the thermo-electric cooler, CCD electronics, spectrometer electronics, the data processing unit (DPU), and the low-voltage power supply. The ChemCam MU is contained in 
Fig. 2 Overview of ChemCam showing the separate body and mast units with their respective subsystems

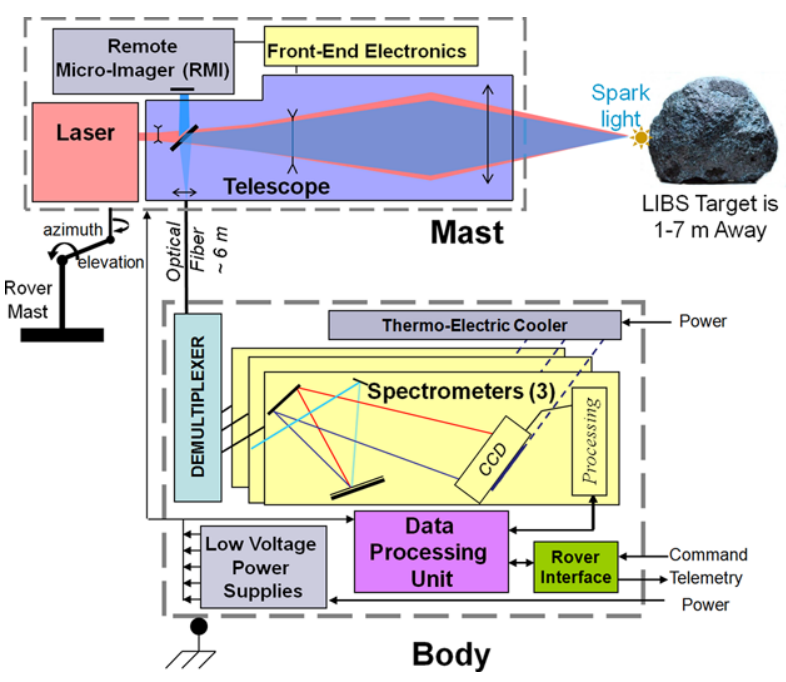

the remote warm electronics box (RWEB) at the top of the rover's mast. The BU is located in the rover body, attached to the rover accommodation mounting plate (RAMP), as described below. Besides the MU and BU, there are two other essential components: A fiber optic cable transmits the plasma light between the MU and BU, and a calibration target assembly (CCCT) is attached to the rover deck. Figure 3 shows a photo of the BU and the CCCT.

We intended to keep the first planetary LIBS instrument as simple as possible and pattern it after laboratory instruments. Most such instruments already use compact spectrometers and optical fibers to feed the light into them. One difference from many laboratory instruments is that ChemCam does not use time-gating to the nano- or microsecond level, which most laboratory instruments achieve with intensified detectors. In the terrestrial environment time gating is needed to reduce the signal from bremsstrahlung. Fortunately, in the Mars environment the effects of pressure broadening, shielding, self-absorption, and bremsstrahlung that typically degrade LIBS performance are greatly reduced (e.g., Knight et al. 2000; Sallé et al. 2005). As a result, there is no significant advantage to time-gating the detector, obviating the need for high-voltage intensifiers on ChemCam.

Table 1 gives the mass and overall dimensions of the four parts of the instrument. Several components typically provided by the spacecraft were added to the MU, including RWEB survival heaters and thermally isolating mounting feet. However, the RWEB box covering the MU and the optical mounting table to which the MU is attached are all considered part of the rover and not part of the instrument. The optical fiber mass does not include cabling supports or other accommodation fixtures.

\subsection{Requirements}

The ChemCam LIBS instrument was designed to obtain major element compositions for rocks and soils within seven meters of the instrument to a relative accuracy of $\pm 10 \%$. The instrument must be capable of removing dust and weathering rinds, and must be able to profile to a depth of $1 \mathrm{~mm}$ into rocks. Table 2 lists the requirements specific to the ChemCam BU, mast-to-body fiber, and rover calibration targets to support these top-level requirements.

The following rationale was used in developing the transmission and wavelength requirements: The optical fiber requirements are mostly capability-driven. This is illustrated by the 

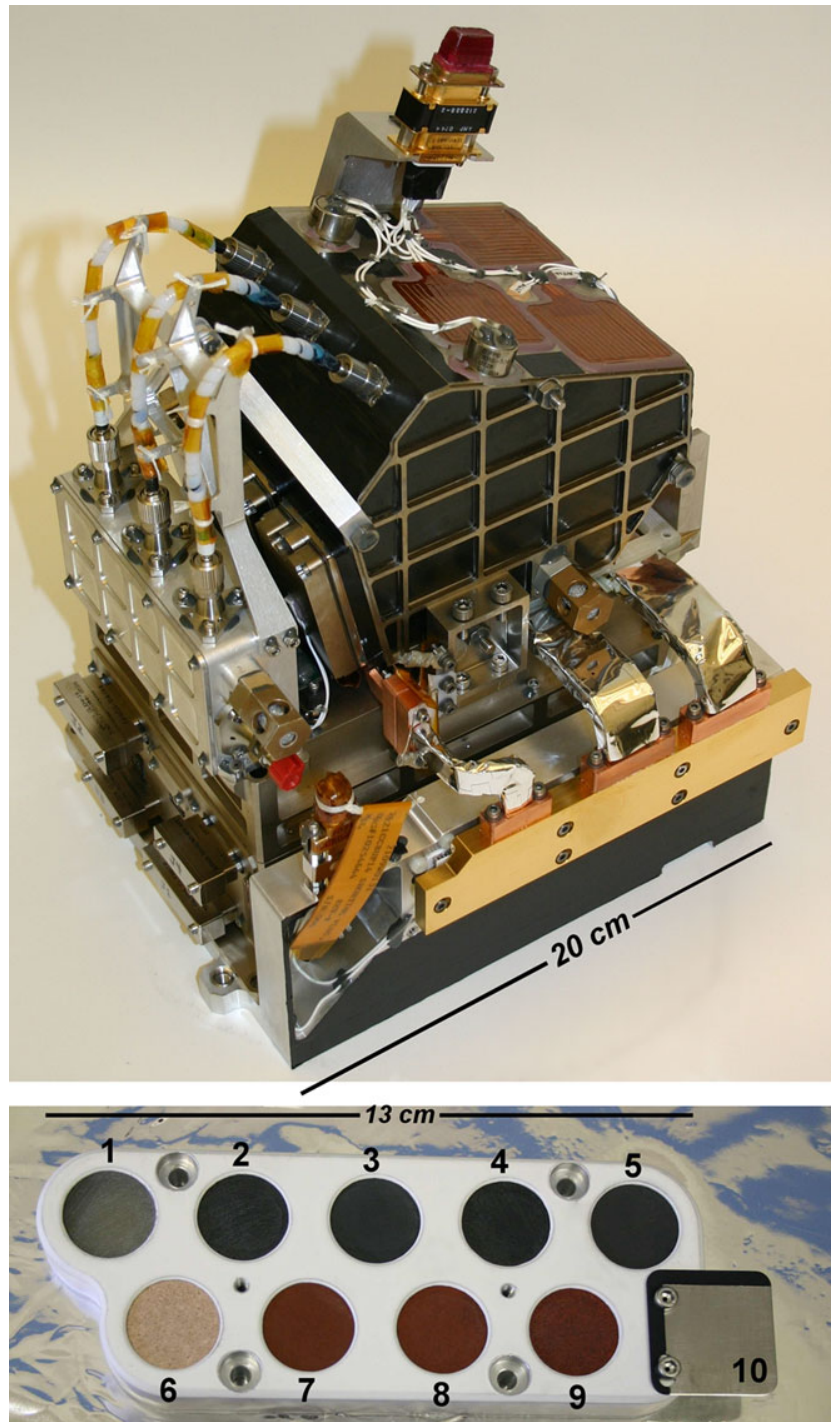

Fig. 3 ChemCam body unit (BU) and on-board calibration target assembly (CCCT). See text and Table 5 for description of each numbered target

Table 1 Masses and dimensions of the different ChemCam sections

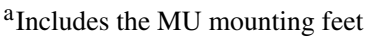

${ }^{\mathrm{b}}$ Includes TEC. Mass of BU without the TEC is $2445 \mathrm{~g}$

\begin{tabular}{lll}
\hline Instrument section & Mass $(\mathrm{g})$ & Dimensions $(\mathrm{mm})$ \\
\hline Mast Unit $^{\mathrm{a}}$ & 5778 & $384 \times 219 \times 166$ \\
Body Unit $^{\mathrm{b}}$ & 4789 & $197 \times 236 \times 154$ \\
Fiber Optic Cable & 63 & $5743 \times 1.4 \mathrm{dia}$ \\
Calibration Target & 161 & $146 \times 51 \times 16$ \\
\hline
\end{tabular}


Table 2 ChemCam body unit, optical fiber, and on-board calibration target functional requirements

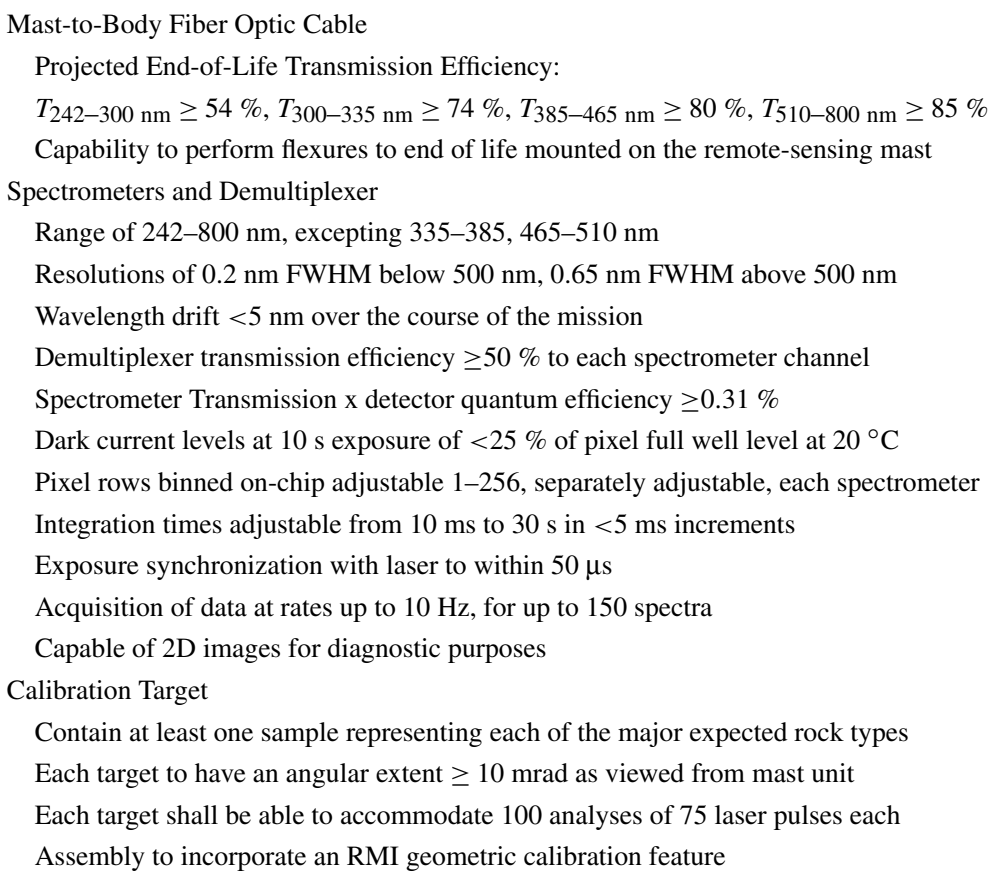

much looser transmission requirements in the UV range than at longer wavelengths. The shortest required wavelength for the LIBS system $(242 \mathrm{~nm})$ was set by the strong desire for carbon detection. A relatively intense carbon emission line exists at $247.8 \mathrm{~nm}$, so the wavelength range requirement was extended to $5 \mathrm{~nm}$ below this, and a very loose wavelength drift requirement of $<5 \mathrm{~nm}$ was set. The long wavelength requirement of $800 \mathrm{~nm}$ was likewise set by the wavelengths of oxygen and potassium emission lines at 777 and at 767 and $770 \mathrm{~nm}$, respectively, which are the strongest emission lines for these elements.

The spectrometer resolution requirements were based on potential interferences for emission lines of significant interest. The LIBS emission lines are optically narrow, with most of them having line widths well below the resolution of the instrument. The density of emission lines is quite high in the UV and near-UV spectral regions, necessitating relatively good spectral resolution in these regions. As examples of separations which drove the requirements, the carbon emission line at $247.8 \mathrm{~nm}$ line must be resolved from a Fe line at $248.3 \mathrm{~nm}$, and the $\mathrm{Sr}$ emission line at $407.8 \mathrm{~nm}$ must be resolved from a Fe line at $407.2 \mathrm{~nm}$. Strong emission lines in the spectral region above $\sim 500 \mathrm{~nm}$ are generally less closely spaced, so the resolution requirement was relaxed in this wavelength region, as specified in Table 2.

The requirements for demultiplexer and spectrometer optical transmission in Table 2 are the result of an evolution of the optics during the first two years of development. At the time of the proposal we knew of no published measurements of the irradiance of LIBS emission lines of geological samples at the planned laser power density. However, within a year the proper irradiance measurements were obtained. A review of optical capabilities and the subsequent spectrometer optical response requirement (specified as spectrometer transmission multiplied by detector quantum efficiency) suggested the need for an optical demultiplexer which was capable of providing at least $50 \%$ of the light to each spectrometer over their 
respective wavelength ranges. In the event that too much light is received, integration over fewer horizontal rows of the two-dimensional (2D) CCD array can be used as an effective aperture adjustment A requirement for longer integration duration capability, for up to $30 \mathrm{~s}$, applies to passive reflectance spectra. A requirement was also added for acquiring 2D CCD images for characterizing hot pixels and checking the alignment of the optical beam. Finally, several basic requirements specify that the spectrometer CCDs must be synchronized with the LIBS laser and that data must be obtainable over a number of spectra (up to 150) at the laser repetition rate of up to $10 \mathrm{~Hz}$. Requirements for the thermo-electric cooler (TEC) will be discussed later.

Table 2 also lists several requirements for the ChemCam on-board rover calibration target assembly. The goal was to have LIBS targets representative of the general types of samples expected on Mars, as captured in the first calibration target requirement listed in Table 2. Because LIBS targets degrade very slowly with repeated use, a requirement was placed on the durability of the targets with respect to laser ablation. Each target is expected to be used approximately once per week, so targets are required to last for at least 100 analyses of 75 laser pulses each for the nominal MSL mission (one Mars year, or nearly two Earth years). The target size requirement was levied by the flight system, based on the expected pointing capability of the mast. The targets are located $1.56 \mathrm{~m}$ from the mast, and are required to be at least $10 \mathrm{mrad}$ in angular extent, leading to an exposed diameter requirement of at least $15.6 \mathrm{~mm}$. The calibration target assembly was also required to incorporate a feature for calibration of the RMI.

\subsection{Development Strategy}

As a novel type of instrument, ChemCam needed a development plan rich with hardware. Three units were built for the MU: an engineering model (EM), an engineering qualification model (EQM), and a flight model (FM). The EM allowed the initial design to be tested for performance. The EQM, which was completely flight-like, underwent qualification level testing while the FM was tested at somewhat lower flight acceptance levels (Maurice et al. 2012). For the body unit, an engineering development unit (EDU) of a single spectrometer was built and it was vibration tested prior to the preliminary design review (PDR), leading to some changes in design for the EM and FM spectrometers. An EDU demultiplexer was also built and tested, which led to significant changes for the EM, and shock testing of it led to additional modifications for the FM.

Regarding the mass of the instrument (Table 1), the BU was initially designed to be very lightweight. The spectrometers were constructed out of Be and the electronics housing was fabricated out of $\mathrm{Mg}$, which is lighter than $\mathrm{Al}$. These account for the relatively light weight of the BU without the thermo-electric cooler. By the time the TEC was added (rationale described later), the MSL project did not need to save mass, so the TEC weighs nearly as much as the rest of the BU.

The ChemCam team at LANL developed the electronic system architecture for the BU. The data processing unit (DPU) was based on a prototype from another program although significant changes were made to accommodate the ChemCam functions. After accommodating these expanded DPU functions, the circuit board design went directly to the EM stage. Two EM low-voltage power supply (LVPS) boards were built using a Los Alamos design. Minor changes were made to the FM, such as additional heat sinking for some of the modules.

LANL and Ocean Optics, Inc. (OOI) collaborated to develop the EM spectrometers and electronics based on the HR2000 spectrometer, a commercial OOI product. LANL made de- 
sign and part changes necessary for the flight environment and built the flight unit. A spaceradiation test failure of the original commercial CCD required a different and more complex detector. This resulted in less commercial heritage in the detector electronics and significantly different designs for the CCD cards and the spectrometer board. The EM spectrometer electronics used mostly non-flight-rated parts, while the FM substituted flight-rated parts. The EM BU is form, fit, and functionally equivalent to the flight unit with a few exceptions; for example, it does not include the TEC. The voltages provided to the EM CCDs are identical for all three detectors, whereas the flight unit accommodates separate voltages for each CCD. Additionally, the demultiplexer is a pre-flight version, and the EM calibration target assembly has different LIBS targets though the rest is identical.

The EM instrument was delivered to Jet Propulsion Laboratory (JPL) and incorporated first into a payload system test bed (PSTB) and later into a vehicle system test bed for development and testing of spacecraft software. The EM was later returned to the instrument team and replaced at JPL with an electronic simulator. Laboratory units of ChemCam have been built and installed at LANL and IRAP. The LANL lab unit uses a newly built BU and the EM MU, while the IRAP lab unit consists of the EM BU and the EQM MU. The lab units allow high fidelity testing and calibration with Mars analog samples during the course of the mission.

\section{Instrument Design}

In this section the instrument is described in detail. The optical components are described first. As mentioned earlier, the MU is described in a companion paper (Maurice et al. 2012), so the description begins here with the optical fiber which optically links the MU and BU. A description of the demultiplexer and spectrometers follows the fiber section. Details of the electronics follow that, and finally the on-board target assembly.

\subsection{Mast-to-Body Optical Fiber}

A custom fiber optic cable (FOC) connects the ChemCam MU to the demultiplexer assembly in the BU. This configuration allows full-range mobility for the rover mast while minimizing actuated mass on it. A single multi-mode fiber conducts the collected light for all three spectral channels to the demultiplexer, where the spectral bands are separated by a series of dichroic filters and fed to their respective spectrometers. Instrument cables were the responsibility of JPL, which in turn contracted to Goddard Space Flight Center to provide expertise and some aspects of the fiber production, while JPL remained in charge overall.

The requirements and design features for the FOC are outlined below:

(1) A fiber length of $5.743 \pm 0.050 \mathrm{~m}$ was required. This length accommodated routing of the fiber from the MU, down through three turns about the elevation mandrel, and three turns through the azimuth twist cap. The fiber turns are loose within the elevation mandrel and twist cap to provide sufficient slack to allow the mast to point over the entire upper hemisphere without exceeding the minimum bend radius for the fiber. The FOC continues down to the bottom of the mast, through the mast deployment joint, and across the top deck to the side of the rover. From there it is routed through a hole in the side of the rover, and then connected to the demultiplexer.

(2) The fiber in the FOC must accept light with a numerical aperture (NA) of at least 0.124. This requirement arises from the design of the light-collecting optics operated over its intended focal range (Maurice et al. 2012). 
(3) The FOC must efficiently conduct light across the instrument's various spectral bands from $242 \mathrm{~nm}$ to $800 \mathrm{~nm}$. The final spectral transmission requirements at end of useful life are given in Table 2.

(4) The FOC must survive temperature extremes of $-130{ }^{\circ} \mathrm{C}$ to $+110{ }^{\circ} \mathrm{C}$, experienced during cruise to Mars and the planetary protection bake, respectively and operate from $-90{ }^{\circ} \mathrm{C}$ to $+50{ }^{\circ} \mathrm{C}$, which bounds the expected thermal extremes of the Martian environment.

(5) A $300 \mu \mathrm{m}$ diameter core is required to accommodate the field of view required for LIBS observations by the telescope when it is focused at various distances. A cladding layer resulting in an overall diameter of $330 \mu \mathrm{m}$ was specified to minimize leakage of light from the conducting core.

(6) A buffer layer of polyimide or acrylate was specified to provide nick protection and mechanical strength to the fiber.

(7) The fiber cable must limit losses to a maximum variability across the spectrum of $\pm 5 \%$ at all combinations of azimuth and elevation positions when installed, and across all operational temperatures.

(8) A maximum radiation-induced attenuation across the spectrum of $-0.3 \mathrm{~dB}$ was allocated given expected cruise and Martian radiation environments.

(9) The fiber cable must comply with additional requirements for vibration survival, outgassing, launch depressurization, pyro-shock, and motion life testing.

The characteristics of different fibers and fiber/jacket combinations were evaluated experimentally to select the optimal fiber and jacket combination. The test fiber samples consisted of (1) a $6.0 \mathrm{~m}$ bare (unjacketed) 0.22 numerical aperture (NA), high oxygen and hydrogen $(\mathrm{OH})$ step-index fiber from Polymicro's FVP line, (2) a $6.0 \mathrm{~m}$ bare $0.22 \mathrm{NA}$, high-OH stepindex fiber from Polymicro's FDP line, (3) a $5.0 \mathrm{~m}$ bare $0.12 \mathrm{NA}$, high-OH step-index fiber from Nufern, and (4) a $6.1 \mathrm{~m}$ similar sample of Nufern 0.12 NA fiber with a W.L. Gore FON1442 jacket.

Throughput tests were conducted on a system consisting of a combined deuteriumhalogen source, providing signal across the wavelengths of interest. To fill the mode-area of the test fiber the coupled output of the light source was inserted into a small integrating sphere to which a $300 \mu \mathrm{m}$ core, $0.22 \mathrm{NA}$, low-OH optical fiber was connected at a port 90 degrees from the input port. This fiber fed a $0.3 \mathrm{~m} \mathrm{~F} / 4$ scanning monochromator connected to a set of order sorting filters and a UV-enhanced silicon photomultiplier tube (PMT).

Baseline spectral measurements $(B(\lambda))$ of the test system were taken to record the nominal response of the system without a test fiber. The PMT voltage, integration time and number of co-added samples were varied over selected spectral ranges to account for spectral variations in the light source, PMT responsivity, spectral characteristics of the order sorting filters, and system throughput efficiency. These parameters were programmed into the monochromator scanning software to keep the signal from saturating the PMT at any point in the spectrum and to boost the signal in low-response regions of the spectrum. Dark scans $(D(\lambda))$ of the experimental apparatus were then taken with the light source switched off to record the spectral effects observed from incident room light and dark current from the PMT. Finally, signal scans were taken $(S(\lambda))$ with the fiber under test inserted between the integrating sphere and the fiber connecting to the monochromator to measure its spectral insertion loss. The spectral throughput of the fiber $(T(\lambda))$ was then calculated from these three data sets according to the equation

$$
T(\lambda)=\frac{S(\lambda)-D(\lambda)}{B(\lambda)-D(\lambda)}
$$


Fig. 4 Throughput of two optical fibers, \#221 and 223, before, at the mid-point of, and after concluding over 2000 thermal cycles. Essentially no change is seen over the course of the test

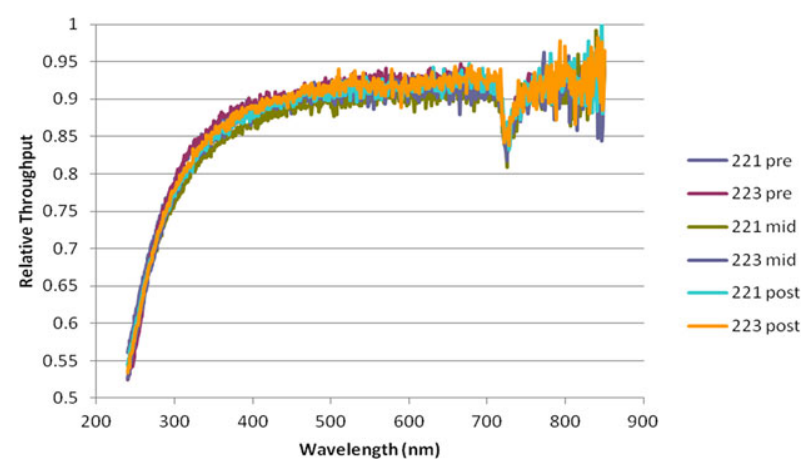

The results led us to select the standard high-OH multimode 0.22 NA fiber (Polymicro FVA 300330500) along with a standard W.L. Gore FON $14421.4 \mathrm{~mm}$ diameter tight-wrapped jacket. This jacket combination consists of a thin buffer layer of ePTFE, a J800 fluoropolymer protective layer, a bradded Kevlar layer for strength, and a final outer J800 fluoropolymer layer. Prior to termination, the jacketed fiber was preconditioned with 40 thermal cycles ranging from $-30{ }^{\circ} \mathrm{C}$ to $+110^{\circ} \mathrm{C}$. Both ends of the fiber were terminated with Diamond AVIM 6236.6 flight-qualified connectors.

The final FOC design was subjected to a rigorous, comprehensive flight qualification suite of tests to assure performance of the ChemCam instrument would not be limited by degradation or failure. These steps include Packaging Qualification and Verification (PQV) testing equivalent to 3 martian years of thermal cycling, vibration testing of the cables and connectors, cable thermal cycling, planetary protection high-temperature bake, total radiation dose exposure, attenuation versus motion, attenuation versus temperature, and a rigorous motion life test running the fiber through 3-year's worth of thermal and motion cycles. The FOC performed very well through all of these tests.

Spectral throughput is shown in Fig. 4 for two distinct FOC assemblies (FOC 221 and FOC 223) each of which experienced over 2000 full-range thermal cycles. Throughput of the fibers was measured prior to cycling ("pre"), after 1288 thermal cycles ("mid") and after 2010 cycles ("post"). Overall transmission did not appreciably change over the test, and the fibers performed comparably (within the test apparatus' $\pm 1 \%$ calibration repeatability). Throughout almost all of the visible spectrum, transmission was at or above $90 \%$, and the data show that the fiber optic cable clearly exceeded the throughput requirements across all spectral bands. Additional details are given in Lindensmith et al. (2010) and Roberts et al. (2010).

\subsection{Demultiplexer}

The optical demultiplexer divides the incoming light into three wavelength bands and thereby increases the light available in the appropriate wavelength ranges to the individual spectrometers. The three spectral ranges are given in Table 3, referred to hereafter as ultraviolet (UV), violet (VIO), and visible and near infrared (VNIR). Using a typical beam splitter would result in less than one third of the light going to each spectrometer. The dichroic beam splitter arrangement in the demultiplexer provides almost twice as much in-band light to each spectrometer over what might be expected with a simpler neutral-density beam splitter arrangement. Additionally, the demultiplexer launches the light into fiber bundles which are arranged in a linear geometry at the spectrometer slit so that a much larger fraction 
Table 3 Optical specifications and performance of the three spectral ranges

\begin{tabular}{llll}
\hline Parameter & UV & Violet (VIO) & VNIR \\
\hline Spectrometer range (nm) & $240.1-342.2$ & $382.1-469.3$ & $474.0-906.5$ \\
Demultiplexer transmission $^{\mathrm{a}}$ & $>50 \%$ & $>60 \%$ & $>50 \%$ \\
Fiber bundle configuration & $12 @ 70 \mu \mathrm{m}$ & $12 @ 70 \mu \mathrm{m}$ & $19 @ 50 \mu \mathrm{m}$ \\
Slit width & $21 \mu \mathrm{m}$ & $25 \mu \mathrm{m}$ & $21 \mu \mathrm{m}$ \\
Spectrometer mirror reflectance & $>96 \%$ & $>98 \%$ & $>98 \%$ \\
Grating ruling & $2400 \mathrm{lpmm}$ & $2400 \mathrm{lpmm}$ & $6001 \mathrm{pmm}$ \\
Grating efficiency & $60-73 \%$ & $48-55 \%$ & $55-72 \%$ \\
Spectral resolution FWHM & $0.15 \mathrm{~nm}$ & $0.20 \mathrm{~nm}$ & $0.61 \mathrm{~nm}$ \\
CCD QE & $62 \% @ 300 \mathrm{~nm}$ & $94 \% @ 400 \mathrm{~nm}$ & $93 \% @ 650 \mathrm{~nm}$ \\
CCD imaging full wells & $99 \mathrm{k} \mathrm{e}^{-}$ & $96 \mathrm{ke}^{-}$ & $85 \mathrm{ke}^{-}$ \\
CCD serial full wells & $255 \mathrm{k} \mathrm{e}-$ & $190 \mathrm{k} \mathrm{e}^{-}$ & $216 \mathrm{ke}^{-}$ \\
Gain & $4.03 \mu \mathrm{VV} / \mathrm{e}^{-}$ & $4.35 \mu \mathrm{V} / \mathrm{e}^{-}$ & $4.21 \mu \mathrm{V} / \mathrm{e}^{-}$ \\
Noise @ 0 ${ }^{\circ} \mathrm{C}$, std. dev. & $1.01 \mathrm{DN}$ & $0.73 \mathrm{DN}$ & $0.71 \mathrm{DN}$ \\
\hline
\end{tabular}

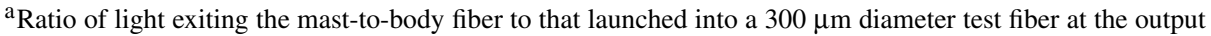
positions

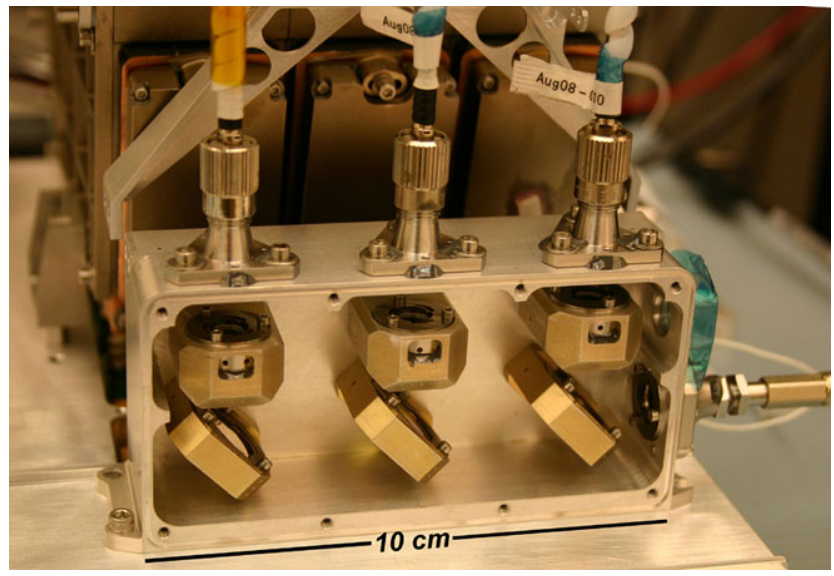

Fig. 5 View of the interior of the optical demultiplexer. Light enters from the fiber bulkhead at right and exits via the three fiber bundles at the top of the image. Dichroic mirrors (right two) and an aluminized mirror (left) are mounted at an angle with focusing lenses positioned above them

of the light can enter the spectrometers. Overall, the combination of the demultiplexer and fiber bundles increases the light transmitted into each spectrometer by nearly an order of magnitude.

The demultiplexer is shown in Fig. 5. The unit consists of an optical fiber bulkhead, which mates to the incoming fiber from the mast, a collimating lens positioned nearly flush with the wall and within the bulkhead mount, dichroic mirrors to separate first the UV and then the VIO ranges, an aluminized mirror at the left side to reflect the remaining light, focusing lenses, and fiber bulkheads to connect the output fibers. An aspheric lens designed to cover the entire $240-800 \mathrm{~nm}$ wavelength range is used as the collimating lens. Its focus 
was optimized during alignment by adjusting the distance of the fiber tip from the lens using a screw motion. Separate lenses were designed to focus light into the fiber bundles at the top of the unit. These lenses were optimized for each of the spectral ranges that were covered. The UV band used a $10 \mathrm{~mm}$ diameter fused silica singlet supplied by JPL. The VIO band used a fused silica aspheric singlet identical to the collimating lens. The VNIR band used a $10 \mathrm{~mm}$ diameter cemented doublet provided by JPL and consisting of F2HT Schott and BK7HT glass with flat surfaces at the interface.

Each focusing lens is securely mounted in a barrel which is inserted into a mount. The lenses were focused by the screwing motion of the barrel. After the unit was focused, the position of each lens was staked in place. Likewise, each of the fiber bundles at the output had a lateral $x-y$ adjustment to optimize the launching of the light. During alignment the bulkheads were held in place by a fixture which had fine $x-y$ adjustments. When the throughput was optimized, the bulkhead was secured in place with fastening screws and was staked (epoxy beads are visible in Fig. 5). The demultiplexer is vented through a $15 \mu \mathrm{m}$ sintered stainless steel filter mounted on the side (seen in Fig. 3, but not yet installed in the unit as shown in Fig. 5). The body of the demultiplexer is $114 \times 51 \times 32 \mathrm{~mm}$, not counting fiber bulkheads. The demultiplexer mirrors were manufactured by Materion Barr. Transmission of the UV dichroic is $<3 \%$ at the upper cutoff of the UV spectrometers, and is $>90 \%$ above $382 \mathrm{~nm}$, the lower cut-off of the VIO spectrometer. The VIO dichroic transmits $<1 \%$ at $463 \mathrm{~nm}$, but transmits $6 \%$ at $469.3 \mathrm{~nm}$, the upper cutoff of the VIO spectrometer. This dichroic transmits $50 \%$ at $480 \mathrm{~nm}$, but does not achieve $>90 \%$ transmission until $504 \mathrm{~nm}$, limiting the utility of the VNIR spectrometer below this range.

Light exiting the demultiplexer is launched into three fiber bundles consisting of either 12 fibers with $70 \mu \mathrm{m}$ cores or 19 fibers with $50 \mu \mathrm{m}$ cores. The configurations of the bundles are given in Table 3 . The fibers are arranged in a closest-packed circular pattern at the exit from the demultiplexer and are arranged in a linear pattern at the entrance to the spectrometers (Fig. 6). The bundles were built by CeramOptec and use ferrule coupling (FC) connectors at both ends. The bundles are housed in Teflon tubes which are supported by an additional spiral wrapped layer of Teflon (Fig. 5). The bundles are held in place between each end by an aluminum cable router, which is a set of braces spanning the demultiplexer and the spectrometers to prevent the fiber bundles from bending excessively during vibration.

\subsection{Spectrometers}

Light is introduced into the spectrometers through a $1 \mathrm{~mm}$ tall slit attached to the end of the fiber bundles. The slits, illustrated in Fig. 6, were obtained from National Aperture as $13 \mu \mathrm{m}$ thick stainless steel disks; the slit widths are given in Table 3. The fiber bundles were characterized, and the ones with the highest transmission were selected to have slits installed, after which the transmission was checked again. Additional units underwent thermal cycling to ensure their long-term survival.

The ChemCam spectrometers are a crossed Czerny-Turner design with focal lengths of $102 \mathrm{~mm}$ using spherical-surface mirrors resulting in a magnification of 1.0. Figure 7 shows the optical bench design with a ray trace superposed. The optical design yields a vertical spread of the slit image, which is collected over approximately 150 pixel rows $(\sim 2 \mathrm{~mm})$ on the detector. The collimating mirror is $25 \mathrm{~mm}$ diameter. The camera (focusing) mirror size was increased in the spectral direction relative to the commercial design to be a 1:1.5 rectangle to minimize vignetting. Optical parameters and performance figures are given in Table 3. The spectrometer mirrors were fabricated of SFL57 glass, and use dielectric coatings to maximize the reflectivity, averaging $>96 \%$ in the UV and $>98 \%$ in the VIO and 
Fig. 6 Spectrometer aperture slit plate before (top) and after (bottom) installation on fiber tip
Fig. 7 Spectrometer optical bench with ray trace superposed
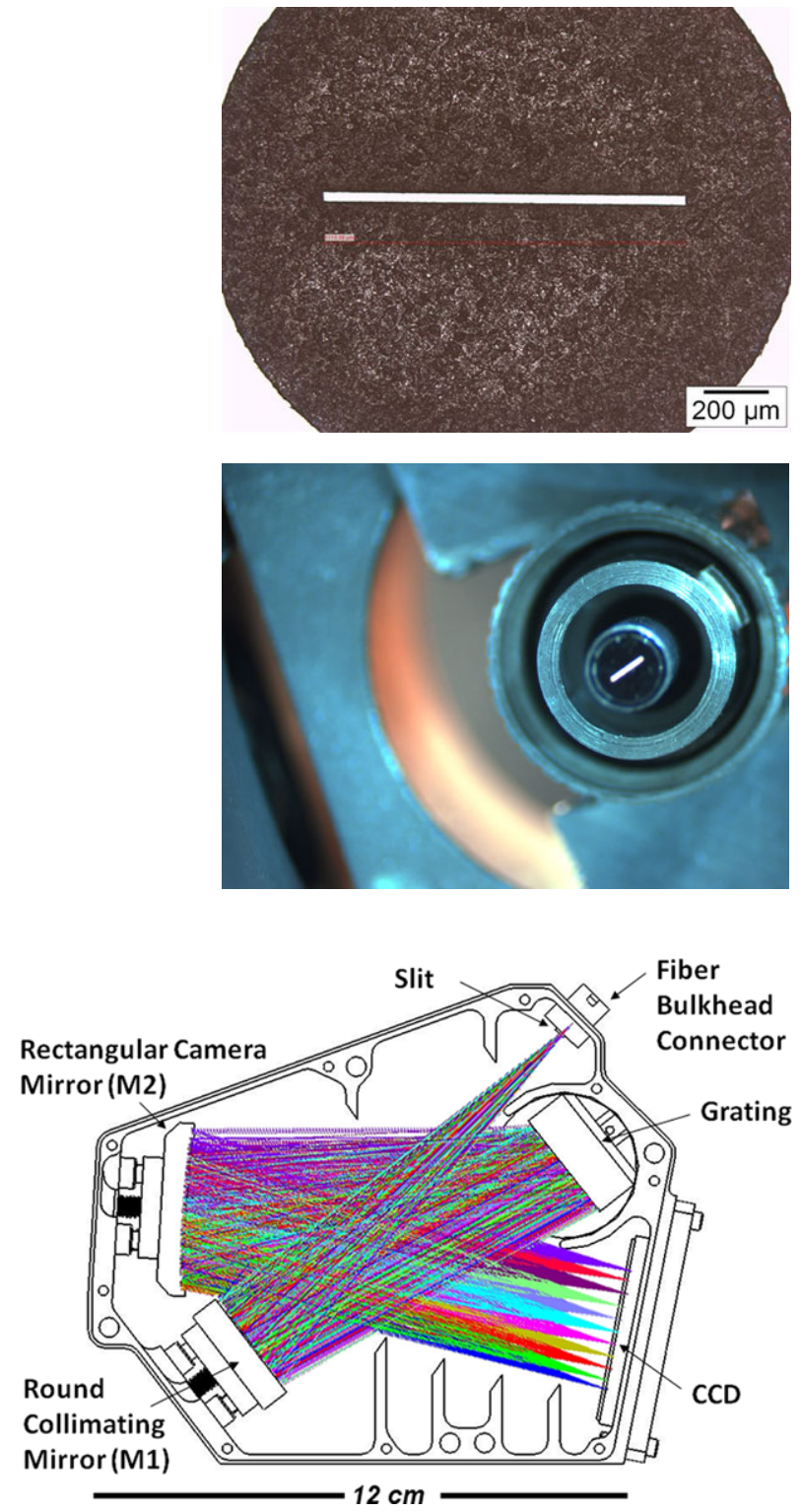

VNIR ranges. The holographic gratings for the UV and VIO spectrometers have 2400 line per mm (lpm), while the VNIR grating has $600 \mathrm{lpm}$. Table 3 shows that each unit meets its spectral range requirement and the VNIR unit actually covers a much larger range, which is useful for recording additional emission lines. With these ranges, and given that each CCD has 2048 pixels across the active area, the UV spectrometer covers 20 pixels per nm, the VIO spectrometer covers 23 pixels per $\mathrm{nm}$, and the VNIR spectrometer has 4.3 pixels per $\mathrm{nm}$, in each case, averaged across their spectral ranges. The full-width half maximum (FWHM) resolution requirements given in Table 2 correspond to, at minimum, 2.8, 4.0, and 4.0 pixels for the VNIR, VIO, and UV ranges, respectively. The spectral resolution obtained at room temperature upon final alignments is shown visually in Fig. 8. Looking at panels (a)-(c) of 

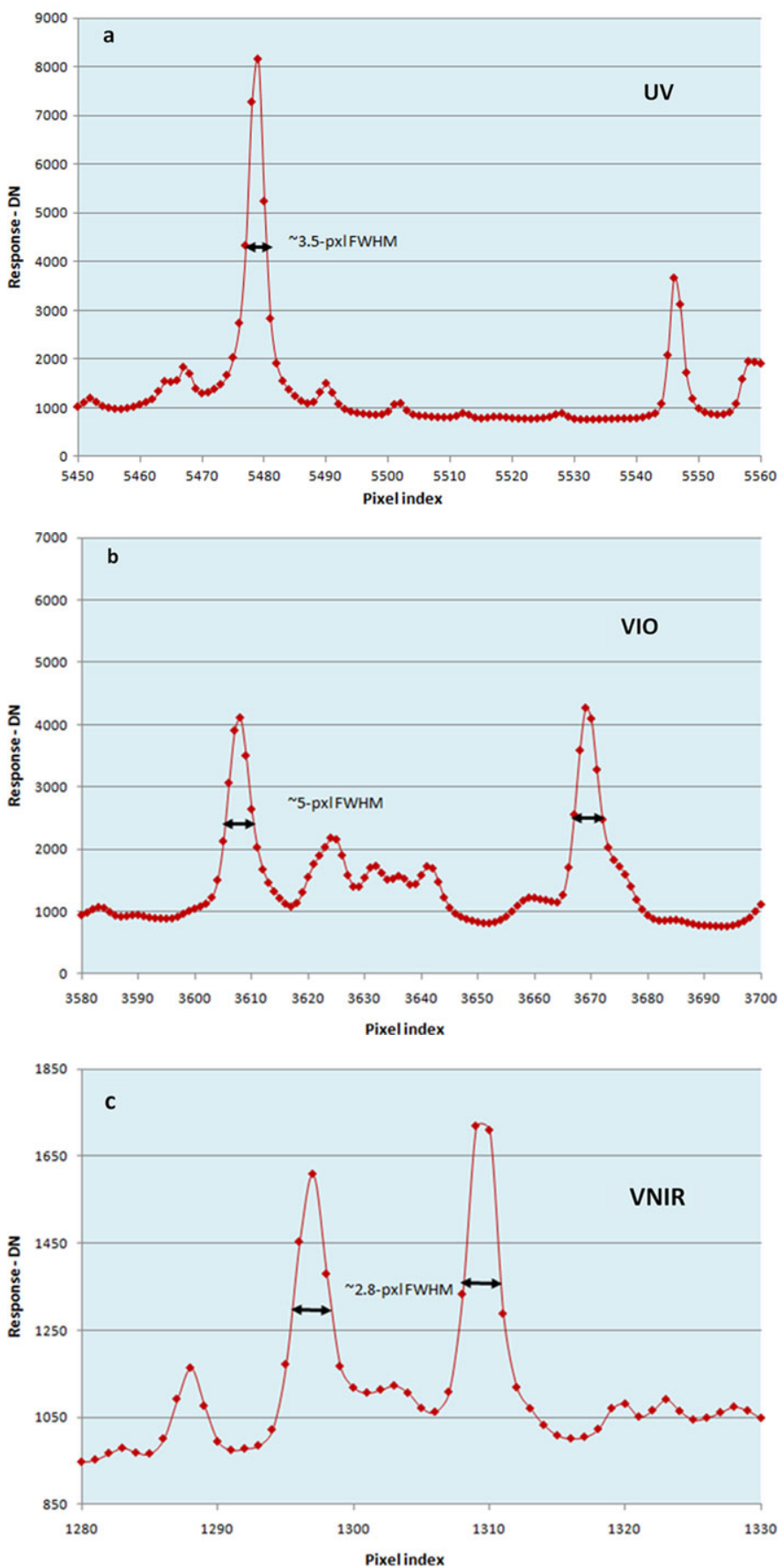

Fig. 8 Spectra of titanium showing the resolution for the UV (a), VIO (b) and VNIR (c) spectrometers at room temperature. The change in resolution as a function of temperature (d) and pixel drift (e) are also shown 

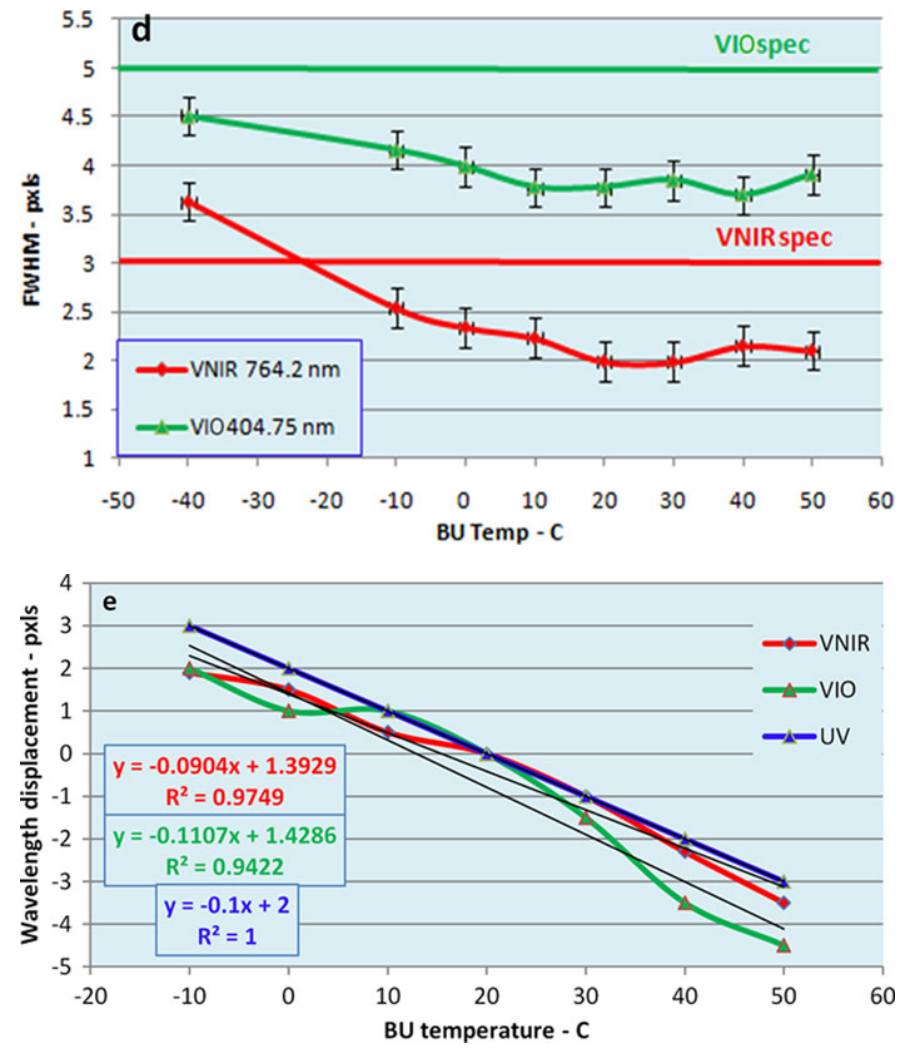

Fig. 8 (Continued)

Fig. 8, one can see that the spectrometers meet this requirement at room temperature for the selected regions shown. Panel (d) shows the resolution stability against temperature for the VIO and VNIR spectrometers. While the required thermal range for performance is from -40 to $+50{ }^{\circ} \mathrm{C}$, the $\mathrm{BU}$ is mounted to the RAMP, which is expected to be between 5 and $35{ }^{\circ} \mathrm{C}$, over which the resolution is virtually invariant. The UV resolution easily met the requirement over the entire thermal range plotted for the other spectrometers in Fig. 8. Finally, the pixel drift with temperature is plotted in Fig. 8e, showing that the UV and VIO spectrometers meet the requirement by over an order of magnitude, while the VNIR unit also easily meets the requirement. Over the expected operation temperature each spectrometer varies by only about three pixels, which is calibratable to a fraction of an Angstrom by making comparisons with reference spectra.

Surface coatings on each E2V CCD (described below) optimize their responses to their specific ranges, utilizing a commercially-available enhanced UV coat for the UV range, an enhanced broadband coat for the VIO range, and a custom coat for the VNIR range. The factory-measured quantum efficiencies are given in Table 3. The main reason for the custom VNIR coat was to minimize etaloning (rapidly varying response as a function of wavelength) resulting from constructive and destructive interferences within the detector itself, which became significant at wavelengths longer than $450 \mathrm{~nm}$. Therefore a graded coating was designed to match the wavelength for each column of pixels. The peak-to-valley quantum efficiency response was required to vary by less than $\pm 2 \%$ of the amplitude up to $777 \mathrm{~nm}$ 
and the actual performance is $\pm 1.3 \%$. The post-burn-in quantum efficiency for the VNIR $\mathrm{CCD}$ is $93 \%$ at $650 \mathrm{~nm}$, dropping to $38 \%$ at $900 \mathrm{~nm}$.

The commercial spectrometer optical bench was modified to reduce mass, and the flight housings were made of $\mathrm{Be}$ to save weight while retaining stiffness and reducing thermal sensitivity. The Be was $\mathrm{Ni}$ coated to reduce human exposure to $\mathrm{Be}$, and flat black aeroglaze Z306 was applied to the interior surfaces to minimize reflections. The three spectrometers were stacked without lids between the units, to save mass. The units are held together with four Ti bolts which run through all three units. The spectrometers are vented through a filter identical to that on the demultiplexer. A hole in the interior dividers between spectrometers allows them to breathe through the same filter. The mirrors are mounted with the same spring and ball-tip three-point adjustment used in the commercial spectrometers. Based on the vibration test on the EDU, the grating mounts were modified to have this same mounting design. The FM gratings were thinned to $6.3 \mathrm{~mm}$ to accommodate this change.

The three spectrometers are together mounted on three feet attached to the electronics box. A survival heater is mounted on the spectrometers to maintain a temperature above $-55^{\circ} \mathrm{C}$. While the spectrometers were designed to meet overall specifications (spectral range, resolution, transmission, drift) over the entire thermal range of the RAMP, which is -40 to $+50{ }^{\circ} \mathrm{C}$, the CCD noise levels would be too high at the upper end of the RAMP thermal range to accommodate LIBS analyses. Moreover, the RAMP temperatures are predicted to be $>20^{\circ} \mathrm{C}$ at all times of the day at the Gale landing site. Three features improve the thermal environment of the spectrometers. The first is the use of titanium, a poor thermal conductor, for the mounting feet, which increases the thermal isolation of the spectrometers. Secondly, the side of the spectrometers facing the rover wall was covered in black Kapton tape, and a patch of black kapton tape was added to the interior wall of the rover to facilitate thermal transfer between the two surfaces, further cooling the spectrometers in the morning hours until the rover wall is warmed by direct sunlight. Most importantly, the thermo-electric cooler described below was added to thermally accommodate the CCDs.

\subsection{Thermo-electric Cooler}

As ChemCam was nearing completion, MSL system engineers determined that the spectrometer CCDs and the MU were not within their operating temperature ranges at the same time of day during most of the planned mission operation scenarios. The strongly preferred operation scenario for the mast is to use it some hours post-sunrise after it has been warmed by the Sun, with optional additional electrical heat; however, the CCDs in the rover body are too warm to operate after several hours past sunrise without active cooling. A joint JPL/LANL engineering team addressed this issue and the result was a system to cool the CCDs using three thermo-electric cooler units (TECs) added to the BU prior to installation in the rover. The requirement was to bring the BU, and specifically the CCDs, into a suitable operating temperature range $\left(<0{ }^{\circ} \mathrm{C}\right.$ for at least 2 hours in the predicted summer worst hot operating case, and longer for other seasons) at the same times of day as the MU to enable all the planned ChemCam surface operations. An implementation was developed which minimized the impact on the existing BU and rover hardware to the maximum extent possible, although some modifications were ultimately required.

The design, shown in Fig. 9, consists of 3 TECs mounted to an L-shaped thermal base plate inserted into the interface between the BU and the RAMP. The TEC base plate serves to conduct waste heat to the rover's heat rejection system, while the vertical portion of the plate positions the TECs such that they can be close to the CCDs and also radiate heat to the adjacent rover external wall. Each CCD package was rebuilt to thermally isolate it from the 
Fig. 9 Thermo-electric cooler (TEC) subsystem. Cf. Fig. 3. The base mounts to the rover, providing a thermal path, as indicated, to the rover's heat rejection system (HRS). The ChemCam body unit electronics box mounts on top of the base plate. The cold frames near the top of the image are attached to the CCDs

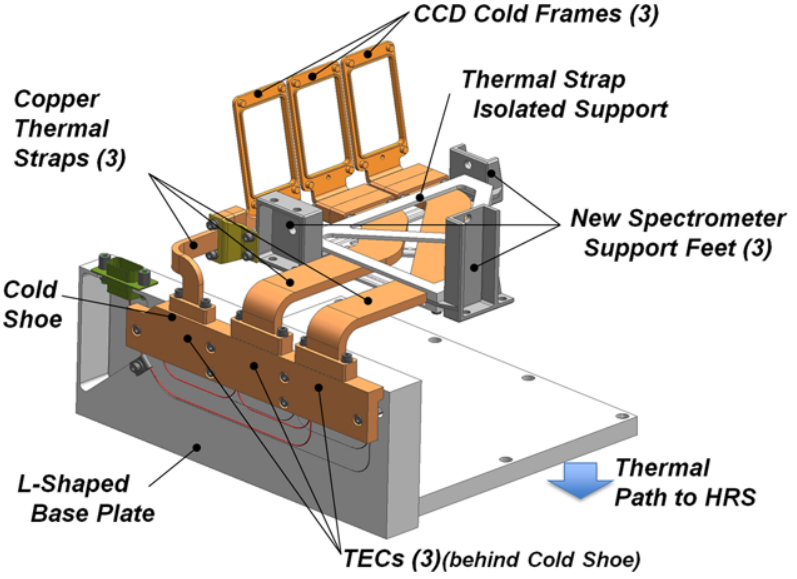

spectrometers, and three custom-designed laminated copper thermal straps provide thermal conduction between each CCD and the TECs. The TECs are wired in series to a common power line that is directly switched by a spacecraft switch, that is, the TEC is independently controlled by spacecraft commands (s-cmds).

The TECs are identical custom-built two-stage devices specifically tailored to meet the thermal performance requirements of this task by Marlow, Inc., which has provided similar flight TECs for several space missions. Each device consists of an array of p- and n-type semiconductor elements sandwiched between ceramic substrates; when current is applied heat is drawn from one side of the device and pumped to the other side. The specific arrangement of the array and the size and number of stages of the device were selected based on the requirements. For this application the primary requirement was to provide a $Q_{\text {lift }}$ of $3 \mathrm{~W}$ per TEC, which extensive thermal modeling indicated was the value required to lower the $\mathrm{CCD}$ temperatures below $0{ }^{\circ} \mathrm{C}$ with this system in all of the required times of day on Mars.

The TECs were mounted as a sandwich between the L-shaped base plate and a copper heat spreader (the "cold shoe"); the entire sandwich was held in place by an array of titanium bolts (Fig. 9). Indium foil was used in every TEC-to-metal interface to ensure good thermal contact between each TEC and the plate and shoe. From the cold shoe the copper thermal straps provided by Space Dynamics Laboratory were routed to the CCDs. The straps and their low-conductance support brackets were intended to generally fit within existing gaps between body unit components and to use existing bolts and bolt locations. However, to provide adequate clearance for the new brackets and straps, new spectrometer mounting feet were designed and built by LANL.

The existing CCD packages required some modification to enable cooling. Specifically the CCDs had to be thermally isolated from the spectrometers and a thermal conducting plate in the shape of window frames had to be added to provide a thermal path out to an interface with the thermal straps, all while maintaining the CCDs at the spectrometer focus and maintaining the package's structural integrity through the same existing bolted interfaces. The LANL engineering team successfully designed and implemented these changes in close consultation with the rest of the joint JPL-LANL TEC design team to ensure the compatibility with the system.

Because the integrated ChemCam BU had already completed its environmental testing, it was necessary to establish that the new design would not adversely impact the existing hardware and that it pass the additional environmental tests required after the new cooling system 
was integrated with the BU. Throughout the design, fabrication, and testing of this system, detailed thermal and mechanical modeling was critical to ensure that the integrated thermal and structural/dynamic performance had sufficient margins to allow the system to be implemented in the short period of time available. JPL thermal and structural engineers worked closely with analysts at Applied Sciences Lab (thermal) and ATA engineering (mechanical) to understand the designed performance at every step of the way. Most significantly, the final tested thermal performance of the modified system was well within predicts and the mechanical system passed all instrument and spacecraft level tests with no issues.

\subsection{Electronics}

\subsubsection{CCDs and Front-End Electronics}

ChemCam uses three identical CCD cards, one for each spectrometer. Figure 10 shows the block diagram of the CCD card, which provides the first stage analog signal amplification, supplies some of the required CCD voltages, and interfaces electrically to the CCDs. The spectrometers were required to be thermally isolated from the rest of the $\mathrm{BU}$, and these separate cards accommodate this isolation using flex circuits for connection to the larger spectrometer board in the BU electronics box.

The spectrometers employ E2V 42-10 back illuminated $2048 \times 515$ pixel CCDs that are operated in low-noise advanced inverted mode. Each pixel is $13.5 \mu \mathrm{m}$ square, for an image area of 27.6 by $6.9 \mathrm{~mm}$. The grade-zero (highest quality) units used in ChemCam were offthe-shelf in terms of specifications with the exception of the anti-etalon coating mentioned above for the VNIR CCD. The full well levels are given in Table 3.

The CCDs underwent normal factory testing provided for commercial units plus one hundred thermal cycles between $-55^{\circ} \mathrm{C}$ and $+70{ }^{\circ} \mathrm{C}$ and a 72 hour burn-in at $125{ }^{\circ} \mathrm{C}$, completed at the factory prior to delivery. The units underwent characterization at JPL for

Fig. 10 Block diagrams of CCD board (a; one of three identical) and spectrometer board (b)

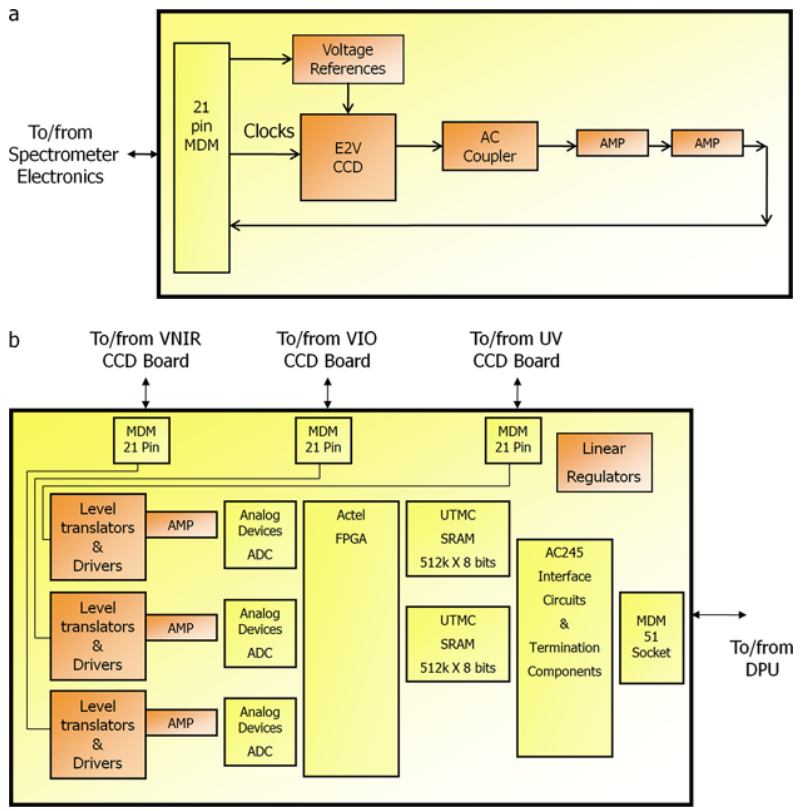


dark current, gain, full well, optimal operating voltages, and any gross anomalies. Because of differences in clock speeds between the initial characterization and flight operation the units had to be tested again with the flight electronics in a socket prior to soldering them to the boards.

\subsubsection{Spectrometer Electronics}

The spectrometer electronics board operates the CCDs used by the three LIBS spectrometers. Figure 10 shows a block diagram of the board. The functions include providing proper reference voltages to drive the CCDs, operating the CCDs, receiving the data from the CCDs, converting the signals to digital data, and passing the data to the DPU.

Reference voltages are provided separately to each CCD on the FM, which allowed preflight adjustment as required to optimize performance. Adjustments are provided for the vertical and horizontal clock speeds as given in Table 4 . These are made via commands sent to the spectrometer field-programmable gate array (FPGA) where the CCD clocking control is performed. Nominal operation for 1D LIBS spectra uses a $56 \mathrm{kHz}$ vertical transfer rate and a $550 \mathrm{kHz}$ horizontal transfer rate, as these appear optimal in terms of overall noise performance. The total transfer time for this setting is a maximum of $\sim 13 \mathrm{~ms}$ if all rows are summed.

When power is applied to the spectrometer board, all three CCDs are turned on but are not clocked. This saves power while warming up the CCDs to equilibrium temperature. Even though nominal operation uses all three CCDs, power to each unit is controlled separately in case of a failure of one, with an accidental exception: the UV and VNIR combination cannot be operated without the VIO spectrometer. The exact timing of each analog to digital (A-D) conversion can be adjusted with a parameter sent to the spectrometer board FPGA, allowing optimization of performance. The clocking parameters (horizontal, vertical and digitizing sample point) are the same for all three CCDs.

Table 3 gives the gain levels used on the A-D converters. After observing the typical signal and noise levels, the gain was set so that the CCD serial full well levels are approximately

Table 4 CCD vertical and horizontal transfer rate settings (V_CLK_SEL and CLK_SPEED, respectively)

\begin{tabular}{lll}
\hline Setting & Vertical $(\mathrm{kHz})$ & Horizontal $(\mathrm{kHz})$ \\
\hline 0 & 83 & 750 \\
1 & 67 & 550 \\
2 & 56 & 434 \\
3 & 48 & 359 \\
4 & 42 & 306 \\
5 & 38 & 266 \\
6 & 34 & 236 \\
7 & 31 & 212 \\
8 & $(\mathrm{n} / \mathrm{a})$ & 192 \\
9 & $(\mathrm{n} / \mathrm{a})$ & 176 \\
10 & $(\mathrm{n} / \mathrm{a})$ & 162 \\
11 & $(\mathrm{n} / \mathrm{a})$ & 150 \\
12 & $(\mathrm{n} / \mathrm{a})$ & 140 \\
13 & $(\mathrm{n} / \mathrm{a})$ & 131 \\
14 & $(\mathrm{n} / \mathrm{a})$ & 123 \\
15 & $(\mathrm{n} / \mathrm{a})$ & 116 \\
\hline
\end{tabular}


$10 \%$ beyond the overflow level of the 14-bit digitizer in order to maximize the sensitivity of the detectors.

\subsubsection{Data Processing Unit (DPU)}

The DPU carries out the functions of taking commands from the rover, performing these commands, storing the data, and sending the data to the rover. In addition, the DPU sends commands to the MU and receives signals and data back in return. A block diagram of the DPU architecture is shown in Fig. 11.

The DPU communicates with the MU through two communication links. Commands and telemetry data between the BU and MU are transferred using a programmable universal asynchronous receiver-transmitter (UART) link which can operate at 4 different speeds: 9.6, $19.2,38.4$, and 115.2 kbaud. Second, images taken by the RMI are sent from the MU to the BU using a low voltage differential signal (LVDS) high speed serial link (HSS) at $8.25 \mathrm{Mbps}$.

The DPU communicates with the rover through a 2-way LVDS HSS link. Command data are transmitted from the rover to the BU at $4.125 \mathrm{Mbps}$. Telemetry data are sent from the body unit to the rover at $8.25 \mathrm{Mbps}$.

The DPU contains a UTMC 80C196 microcontroller, which is supported by two Actel FPGAs. The Micro FPGA, contains the microcontroller logic control, the MU UART interface logic, state of health ( $\mathrm{SOH}$ ) interfacing, and a test port used only during instrument development. The second FPGA, called the Memory FPGA, handles the interfaces to the six megabyte DPU data memory bank, the spectrometer interface, the MU HSS link and the rover HSS links. Program memory is redundantly stored in two banks of EEPROM. One bank is hardware locked, in that the write line is tied off to the inactive state to guard against memory corruption during flight. The other bank is capable of reprogramming in flight if the

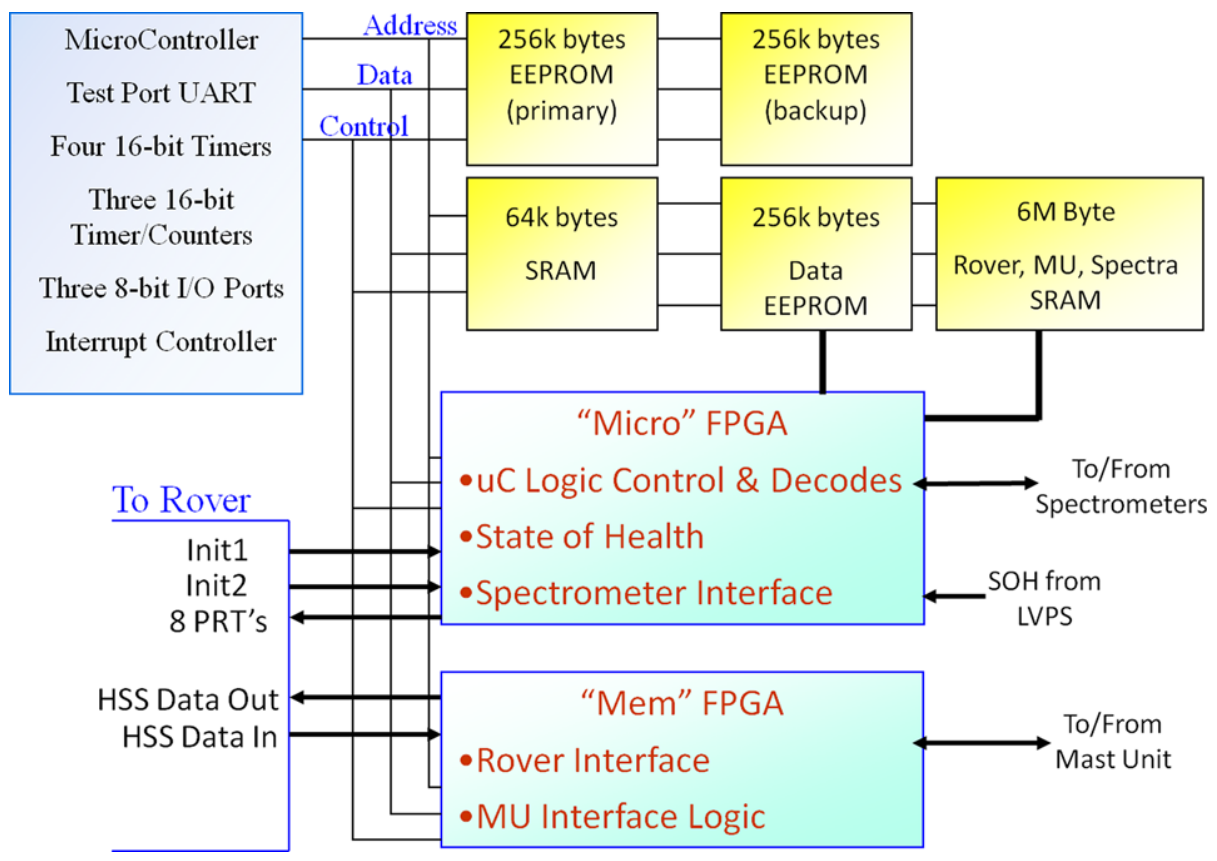

Fig. 11 Block diagram showing the major components of the ChemCam data processing unit (DPU) 
need arises, but is also protected from corruption by the EEPROM's built-in software data protection algorithm. In addition, the DPU also has a bank of scratchpad SRAM and a bank of data EEPROM.

Some changes have been made to the reprogrammable EEPROM bank prior to launch. The handling of interrupts was modified to avoid conflicts which result in approximately $1 \%$ of commands rejected on the locked side. The locked side also operates with an RMI data transmission rate to the rover compute element (RCE) of only $2 \mathrm{MHz}$, while the reprogrammable side can transfer data at up to $8 \mathrm{MHz}$. The locked side also is missing headers on the RMI images, which was fixed in the reprogrammable side.

\subsubsection{Low-Voltage Power Supply (LVPS)}

Figure 12 shows a block diagram of the LVPS. It receives the nominal $28 \mathrm{~V}$ input from the rover, passes it on to the MU, and provides the BU with $+5 \mathrm{~V}$ digital, $\pm 5 \mathrm{~V}$ analog, $+24 \mathrm{~V}$ digital, and $\pm 12 \mathrm{~V}$ analog. Each of these has a maximum capacity of $5 \mathrm{~W}$ except the $+12 \mathrm{~V}$ and $+24 \mathrm{~V}$, which are $2.5 \mathrm{~W}$ maximum. The DPU uses the $\pm 5 \mathrm{~V}$ lines, and the spectrometer board uses all five supplies. Design goals were to maintain noise levels $<5 \mathrm{mV}$ rms and spikes of $<20 \mathrm{mV}$ amplitude. The LVPS board also provides connections for the PRT temperature sensors, two of which are on the spectrometers and two of which are on the CCD board covers. The LVPS board uses commercially available Interpoint power converters with inline filters to achieve its voltage levels. The units are heat sunk to aluminum bars which extend to the edge of the board. The board is housed in a separate compartment accessed from the rover attachment side of the electronics box, where heat is most easily transferred to the RAMP.

Thermal modeling of the LVPS indicated that at the hot qualification temperature limit of $70{ }^{\circ} \mathrm{C}$ ambient, with the board averaging $77^{\circ} \mathrm{C}$, the $+5 \mathrm{~V}$ converter, if operating at $120 \%$ of rated power with a $36 \mathrm{~V}$ bus input, could exceed $125^{\circ} \mathrm{C}$. Additionally, in this scenario, two other converters could exceed $110{ }^{\circ} \mathrm{C}$. While the instrument was successfully tested at these levels, it is not expected to operate near these levels in flight or on Mars.

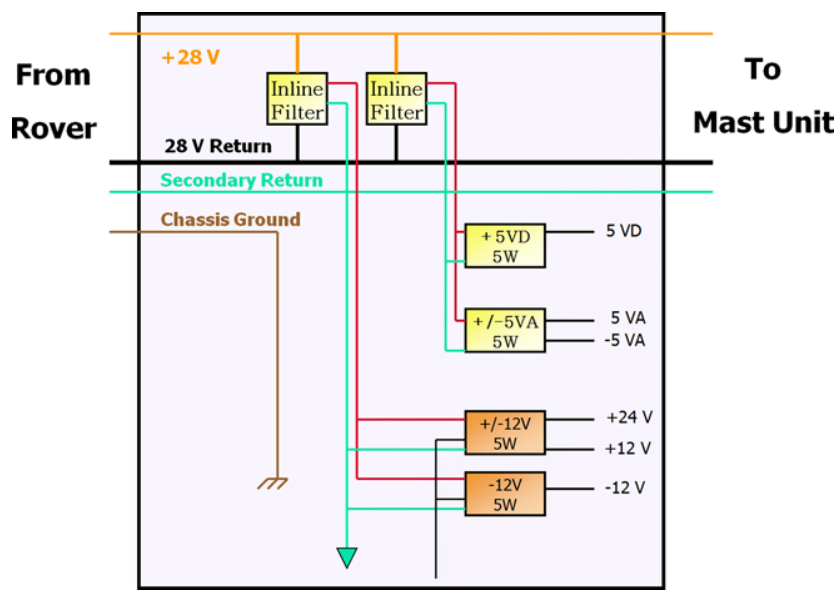

Fig. 12 Block diagram of the ChemCam low-voltage power supply. The orange modules are switched using an inhibit line. $\mathrm{VA}=$ analog voltage; $\mathrm{VD}=$ digital voltage 


\subsection{Rover Calibration Target Assembly}

ChemCam includes a set of LIBS calibration targets on board the rover. Because of various potentially complicating factors for LIBS, including atmospheric pressure, laser power, beam quality, and focusing capabilities - all of which affect LIBS calibration-in-situ calibration using samples of known compositions on board the rover is important. The overall LIBS calibration strategy for ChemCam involves comparisons of rover calibration target measurements on Mars with laboratory measurements of identical samples, backed up by a much larger range of compositions in a large set of laboratory calibration standards, which are used as a training set for multivariate analysis. This approach is necessary because chemical matrix and environmental effects result in complicated relationships between intensity and elemental abundance. A non-linear, emission-line-specific correction for the distance to any given Mars sample relative to the rover calibration target distance then needs to be applied. The calibration work and data treatment will be described further in a forthcoming paper.

The ChemCam rover calibration target assembly is shown in Fig. 3. It consists of eight Mars rock simulants (\#1-4, 6-9), a graphite target (\#5) as a reference for carbon, and a titanium metal plate (\#10) to be used for wavelength calibration and general LIBS diagnostics. Four of the rock simulants are basaltic glass (\#1-4) and four are ceramic mixtures of anhydrite, basalt, and nontronite or kaolinite source clays processed to retain their chemical composition after fabrication into ceramics (\#6-9). This information is summarized in Table 5. Glass and ceramic were used rather than natural rock to aid in both homogeneity on the scale of the laser beam $(\sim 350 \mu \mathrm{m}$ diameter at this distance) and in improved mechanical properties. The eight rock simulants and the graphite consist of cylindrical disks $22 \mathrm{~mm}$ in diameter by $5 \mathrm{~mm}$ thick. They are fitted into cylindrical spaces in the assembly. The disks are pressed from behind with springs against a circular upper lip which exposes the central $18 \mathrm{~mm}$ of the top face of each disk. The size of these openings was governed by the pointing accuracy of the mast, traded against mass limitations for the instrument. The body of the calibration target assembly is made of 6061 aluminum, fabricated at Mount Holyoke College, and is painted white for thermal purposes using the same paint as the rover body.

Three synthetic glasses (Table 5) were fabricated from carbonate, oxide, and sulfur powders by C. Fabre in Nancy, France. After de-carbonation at $800{ }^{\circ} \mathrm{C}$, the powders were melted twice at $1350{ }^{\circ} \mathrm{C}$ for five minutes each. The result of the first melt was ground to $50 \mu \mathrm{m}$ powder before the second melting (Fabre et al. 2011). For the fourth glass target a natural obsidian (macusanite; Pichavant et al. 1988a, 1988b; \#1 in Table 5) was prepared. Eight pieces were cored from a sample at the Museum National d'Histoire Naturelle (MNHN) in Paris. The exposed surfaces were left unpolished to aid in coupling of the laser to the target. Fabre et al. (2011) describes all of the ChemCam igneous glass samples in detail.

Four ceramic targets (Table 5) were fabricated at Los Alamos to broadly simulate sedimentary compositions expected on Mars. Sulfate-bearing lithologies are important on Mars, and particularly at the Gale crater landing site. However, retaining sulfate in fired glass targets is very difficult. To get around this problem it was decided to produce ceramics using a maximum temperature of $800{ }^{\circ} \mathrm{C}$. To simulate Mars sedimentary compositions, combinations of phyllosilicates and basalt powder were combined with dehydrated gypsum to provide the sulfur component. Clay Minerals Society (CMS) source clays were used for the phyllosilicate components. In order to prevent hydration of the clay minerals, which would seriously impact stability of the ceramics, the CMS source clays were pulverized, heated to $1000{ }^{\circ} \mathrm{C}$ and then ground again before being added to other ceramic components and fired at $800{ }^{\circ} \mathrm{C}$. This destroyed the clay mineral structures while retaining all of the chemical 
Table 5 LIBS calibration targets on board the rover

\begin{tabular}{|c|c|c|c|}
\hline Number & Name & Description & Reference \\
\hline $1^{\mathrm{a}}$ & Macusanite & $\begin{array}{l}\text { Natural obsidian glass from the Andes Mountain Range, South } \\
\text { America }\end{array}$ & $\mathrm{b}, \mathrm{c}$ \\
\hline 2 & Norite & Synthetic glass; potential analog of the Noachian crust & $\mathrm{c}$ \\
\hline 3 & Picrite & $\begin{array}{l}\text { Synthetic glass; analog of abundant composition on the martian } \\
\text { surface (e.g., McSween et al. 2006) }\end{array}$ & $\mathrm{c}$ \\
\hline 4 & Shergottite & $\begin{array}{l}\text { Synthetic glass; Mars meteorite-like; resembles Bounce rock } \\
\text { Meridiani Planum (e.g., Zipfel et al. 2011) }\end{array}$ & $\mathrm{c}$ \\
\hline 5 & Graphite & Graphite rod, included for identification of $\mathrm{C}$ emission lines & $\mathrm{d}$ \\
\hline 6 & KGa-2med-S & Ceramic; CMS kaolinite standard mixed with basalt and anhydrite & $\mathrm{e}$ \\
\hline 7 & $\mathrm{NAu}-21 \mathrm{lo}-\mathrm{S}$ & $\begin{array}{l}\text { Ceramic; CMS nontronite standard mixed with basalt and a low } \\
\text { ratio of anhydrite }\end{array}$ & e \\
\hline 8 & NAu-2med-S & $\begin{array}{l}\text { Ceramic; CMS nontronite standard mixed with basalt and a } \\
\text { medium ratio of anhydrite }\end{array}$ & e \\
\hline 9 & NAu-2hi-S & $\begin{array}{l}\text { Ceramic; CMS nontronite standard mixed with basalt and a high } \\
\text { ratio of anhydrite }\end{array}$ & e \\
\hline 10 & Ti plate & $\begin{array}{l}\text { 6-4 titanium alloy; to be used for wavelength calibration and } \\
\text { diagnostic LIBS tests }\end{array}$ & \\
\hline
\end{tabular}

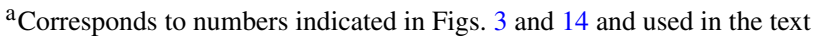

${ }^{b}$ Pichavant et al. (1988a, 1988b)

${ }^{\mathrm{c}}$ Fabre et al. (2011)

dOllila et al. (2011)

eVaniman et al. (2012)

components except $\mathrm{H}_{2} \mathrm{O}$ and $\mathrm{OH}$. Experiments determined that these materials alone, when pressed and fired, were too structurally weak, so $\sim 9 \mathrm{wt}$. $\%$ of lithium tetraborate was added to provide sufficient hardness. The ceramics were fired at the approximate diameter that would be used on the rover. However, the diameter of the KGa-2 ceramic shrank less during firing and had to be machined back down to the proper diameter (from $22.5 \mathrm{~mm}$ to $22.0 \mathrm{~mm}$ ). The ceramic targets and their chemical analyses are described in detail in Vaniman et al. (2009, 2012).

The last two ChemCam LIBS targets are titanium metal and graphite. The Ti target is for general diagnostics and for wavelength calibration because Ti has a large number of emission lines across all of the ChemCam spectral ranges. It was fabricated from a $0.9 \mathrm{~mm}$ thick plate of typical 6-4 alloy which contains approximately $6 \% \mathrm{Al}$ and $4 \% \mathrm{~V}$, the emission lines of which can be seen in its LIBS spectrum. At one edge the plate was thinned on the underside to $250 \mu \mathrm{m}$. A strip on the top surface along the edge was painted flat black (Fig. 3). With its edge hanging over the white body of the calibration target assembly, it makes a contrast strip for use in determining the optical resolution of the RMI. MastCam's onboard calibration target can also be used for RMI calibration.

A graphite disk was included in the assembly to provide a clear spectrum of carbon for unambiguous identification. The $248 \mathrm{~nm} \mathrm{C}$ emission line is surrounded by nearby $\mathrm{Fe}$ peaks. Because of the importance of $\mathrm{C}$ identification for the MSL mission, it was decided to include this sample as part of the calibration target assembly. The graphite was cut from a stock rod without polishing. Initial LIBS spectra suggested the presence of small amounts of impurities. Analyses of a replicate disk with an electron probe using both energy-dispersive 
and wavelength-dispersive spectroscopy (EDS, WDS) indicated that, at least at the surface, impurities were observed including $\mathrm{Fe}, \mathrm{Al}, \mathrm{Ni}, \mathrm{Ti}, \mathrm{V}, \mathrm{Si}, \mathrm{Na}, \mathrm{K}, \mathrm{Ca}, \mathrm{Cl}$. However, because the impurities are minor, they do not interfere with the utility of the carbon standard.

An engineering model (EM) of the calibration target assembly underwent environmental testing prior to building the flight unit. The EM LIBS standards differed in some ways from the flight versions, for example, the EM glasses did not include any minor or trace elements. The ceramics contained lithium tetraborate in several different proportions. The EM underwent vibration testing to qualification levels, then was subjected to $>2000$ thermal cycles at JPL with a $\Delta T$ of $145^{\circ} \mathrm{C}$ at each cycle, some simulating winter $\left(-130\right.$ to $\left.+15^{\circ} \mathrm{C}\right)$ and some summer $\left(-105\right.$ to $\left.+40^{\circ} \mathrm{C}\right)$. Finally it underwent a qualification level shock test, nominally to $4000 \mathrm{~g}$ 's, but up to $8000 \mathrm{~g}$ 's at higher frequencies. Small fissures appeared in some of the ceramic samples during thermal cycling, and one of the glass targets cracked during the shock test. The rear of the glass target had a latent crack visible prior to the shock test. It appeared to have been produced during annealing and to have been filled in by melt material. It was noted that the thermal cycling and the shock test were not performed in the proper order relative to flight, and that in either case there was no risk to the mission, nor any significant risk to the performance of the calibration targets.

A consumption test was performed on one of the EM calibration targets to ensure that they would not be destroyed by the laser before the conclusion of Mars operations. Fifteen thousand laser pulses were trained on an EM shergottite glass disk, and the results are shown in Fig. 13. The sample was moved slightly after each burst of 100 laser pulses to simulate operation on Mars. For actual ChemCam operation the amount of overlap of laser pits will depend on the mast pointing reproducibility. If it is sufficiently high and results in multiple samplings at the same location, a slight repositioning will be requested to produce more scatter in the position of the laser pits.

Because the calibration target assembly is exposed on the surface of Mars, the FM unit was required to undergo a planetary protection bake, which was done at $110{ }^{\circ} \mathrm{C}$ for 68 hours in vacuum. The remove-before-flight cover and packaging foil were baked along with the unit. Upon receipt at JPL the unit underwent a $50{ }^{\circ} \mathrm{C}$ outgassing test using a quartz crystal microbalance held at $-50{ }^{\circ} \mathrm{C}$ to test against a requirement of $300 \mathrm{ng} / \mathrm{cm}^{2} /$ hour. The observed outgassing rate was orders of magnitude below the requirement, consistent with the observations during the planetary protection bake-out.

Bidirectional reflectance measurements of ChemCam calibration target (CCCT) materials from a flight spare assembly, identical to the FM, were performed in the SESE near-IR reflectance laboratory at Arizona State University. The measurements were made in air at ambient room pressure and temperature using an Analytical Spectral Devices (ASD) Fieldspec 3 visible to near-IR spectrometer, operating in the 350 to $2500 \mathrm{~nm}$ spectral range. The

Fig. 13 Engineering model shergottite calibration target (center of image) after 15,000 laser pulses as part of the sample consumption test

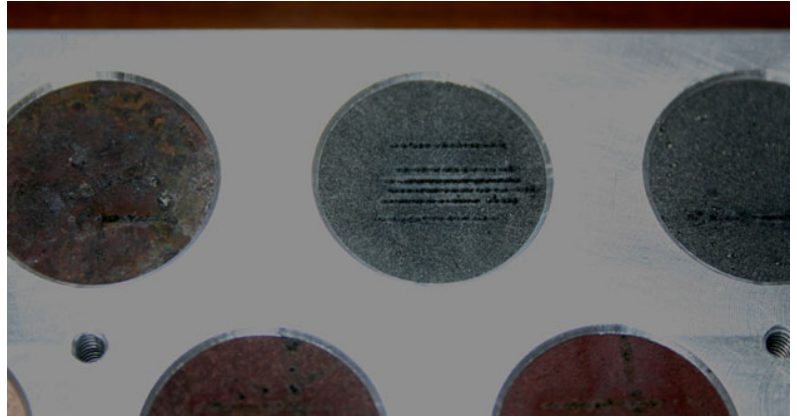


ASD has a spectral resolution of $3 \mathrm{~nm}$ at $700 \mathrm{~nm}, 8.5 \mathrm{~nm}$ at $1400 \mathrm{~nm}$, and $6.5 \mathrm{~nm}$ at $2100 \mathrm{~nm}$. Spectra were also measured of the white paint (defined as sample 11) that was used on the body of the calibration target assembly and on the deck of the rover.

Spectra were acquired in an orientation consistent with the typical geometry of measurements expected on Mars. Specifically, the incidence (illumination) angle was sampled at $0^{\circ}, 30^{\circ}$, and $60^{\circ}$ to simulate a range of solar illumination conditions, using a $50 \mathrm{~W}$ quartzhalogen lamp as the illumination source. The emission (viewing) angle was set to $9.5^{\circ}$ to simulate the viewing geometry of the CCCT materials from the ChemCam instrument. Similar measurements were acquired at the $15^{\circ}$ emission angle expected for viewing of the CCCT from MSL's Mastcam. The emission angles were estimated from engineering drawings of the MSL mast and rover deck to be the approximate angles from ChemCam or Mastcam to the center of the CCCT, relative to the angle normal to the CCCT. The position and angle at which the CCCT was mounted on the rover are described in greater detail in the next section. Raw spectra were calibrated to reflectance by removing background (dark) signal levels and ratioing to the spectra of a calibrated halon reference sample observed at the same viewing geometry.

Figure 14 shows the $350-2500 \mathrm{~nm}$ reflectance spectra of the 9 round samples plus the $\mathrm{Ti}$ plate (sample 10) and white rover paint (sample 11). Each spectrum is offset by the amount indicated to the left of each line, for ease of plotting. Spectra of samples 1 through 5 are relatively dark, and 1-4 exhibit weak, broad bands around 800-1200 nm that could be consistent with their glassy composition containing varying degrees of transition elements like Ti, Cr, and Fe (Fabre et al. 2011). Spectra of samples 6 through 9 have higher reflectances, especially in the near-IR, and have visible to near-IR spectral characteristics dominated by the presence of $\mathrm{Fe}^{3+}$, most likely within crystalline hematite for samples 7, 8, and 9, but possibly within nanocrystalline hematite or another poorly-crystalline ferric oxide, oxyhydroxide, or oxyhydroxysulfate in sample 6. Sample 6 also shows the strongest evidence among all the samples for the presence of $\mathrm{H}_{2} \mathrm{O}$ and/or $\mathrm{OH}$ in the sample based on the presence of a 1900-2000 $\mathrm{nm}$ band. Weaker evidence of hydration is also present in sample 1 . The weak and broad feature seen from about 2100-2300 nm in spectra of samples 1 and 6-9 could also be indicative of the presence of cation-OH absorption in either phyllosilicates or

Fig. 14 Visible to Near-IR bidirectional reflectance spectra of CCCT materials. Samples 1-9 were measured at a typical viewing geometry expected for RMI imaging on Mars: $i=30^{\circ}$, $e=9.5^{\circ}$. Samples 10 and 11 (dashed lines) saturated the signal in that geometry, and so were measured instead at $i=60^{\circ}, e=9.5^{\circ}$. The spectra are keyed to the index of samples shown in the inset (a photo of the CCCT acquired by the MSL Mastcam instrument during pre-flight testing). Spectra are stacked and offset by the amount indicated to the left of each

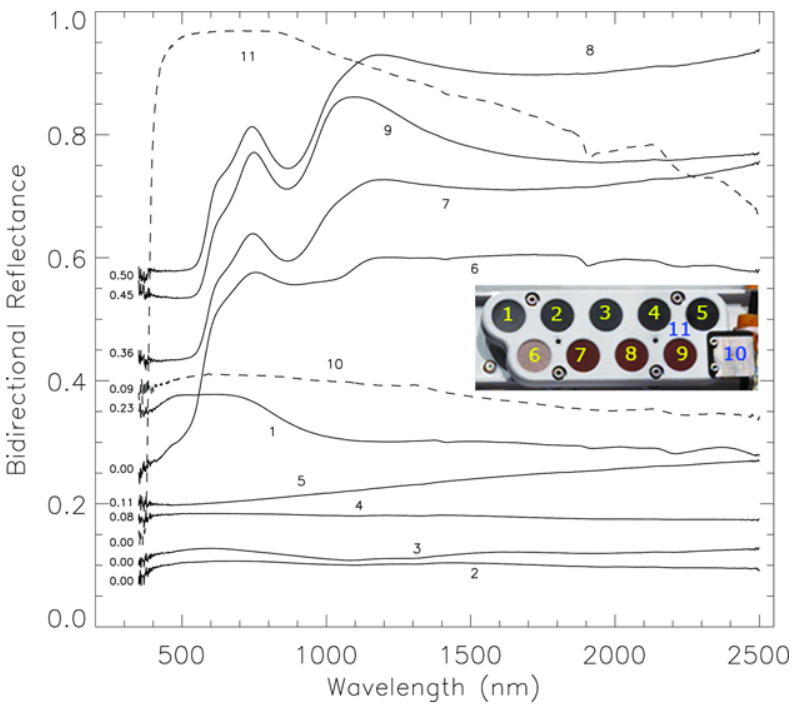


sulfates; the latter is more likely, based on the width of the feature. The Ti plate (10) exhibits a relatively low and slightly blue reflectivity because of its smooth, specular nature (which saturated the measurements in several viewing geometries). In contrast, the white paint on the assembly plate (11) has a very high reflectivity ( $>95 \%$ in the visible) and also saturated the signal at closer to specular geometries. It, too, shows evidence of $1900 \mathrm{~nm}$ and 2100 $2400 \mathrm{~nm} \mathrm{H}_{2} \mathrm{O} / \mathrm{OH}$ features. Spectra acquired at the Mastcam emission angle geometry $\left(15^{\circ}\right)$ are very similar, and do not show any significant differences in adsorption band or spectral slope behavior.

\section{Environmental Testing}

A significant portion of the ChemCam BU and MU environmental testing was conducted separately. Because they are only connected by cables, there was no need to perform vibration and shock testing together. The MU therefore went through these tests prior to delivery from Toulouse to Los Alamos. The BU and calibration target went through individual vibration and shock tests, described below. Each unit also underwent separate thermal testing prior to integration of the two parts of the instrument, due to the fact that each part of the instrument has different survival and operational ranges. After integration the combined instrument underwent initial characterization followed by thermal performance testing and a final LIBS characterization with a larger number of standards before delivery.

The entire BU underwent several rounds of testing because of hardware changes after the MSL launch delay from 2009 to 2011 . Thermal environmental and performance testing was carried out in 2008 and was repeated in 2010 after replacement of the CCDs and demultiplexer and the addition of the TEC. Shock testing was conducted in 2008 and vibration testing was done in 2009, but these were also repeated in 2010 after the same changes mentioned above. The engineering model was not subjected to vibration, shock, or thermal tests before delivery, though components underwent some of these tests.

The BU vibration test used force-limiting acceleration of $7.8 \mathrm{~g}_{\mathrm{rms}}$, one minute per axis. Shock testing was performed to $1400 \mathrm{~g}$ 's for the flight unit. Functional testing was performed before and after each axis by observing a $\mathrm{Hg}-\mathrm{Ar}$ lamp to check spectral resolution and a halogen-deuterium combination lamp to check optical transmission and gain. Electrical continuity was tested for the TEC. Vibration tests before and after thermal and shock testing indicated a change in resonance from 480 to $422 \mathrm{~Hz}$. The cause of this change was never determined, as the unit performed flawlessly in all tests thereafter.

The BU was qualified to -55 and $+70^{\circ} \mathrm{C}$ by operating 24 hours at the cold temperature and 72 hours hot with three starts at each of these operational limits. It was also cycled three times between the two extremes. The TEC was operated for the $0-35{ }^{\circ} \mathrm{C}$ portions of the three cycles, and its thermal performance matched predictions to within one degree. Optical properties of the BU were only checked before and after the test. A change in optical performance of the VIO channel was detected, which led to replacing the demultiplexer focus lens in this band. Thermal testing of the demultiplexer was repeated multiple times thereafter. Overall BU optical properties were tested over the required temperature range during performance testing that also included the MU.

For the calibration target assembly, the EM underwent full testing, including PQV thermal cycling and qualification-level shock testing. The FM assembly only underwent vibration, shock, and sterilization bake-out. It did not go through thermal cycling. The optical fiber did not undergo vibration or shock, but instead went through PQV thermal and RSM motion tests. Radiation exposure testing was done on selected spare components, including CCDs and FOC units. 


\section{Rover Accommodation}

\subsection{Body Unit}

The body unit is mounted upside down to the RAMP along the side of the rover behind the SAM instrument (Mahaffy et al. 2012), as shown in Fig. 15. It is on the same side of the rover as the mast, which helps to reduce the length of the mast-to-body optical fiber. The RAMP, which contains thermal fluid loops from the radio-isotope thermal generator (RTG), provides thermal stability to the instrument, maintaining it within the flight allowable temperature limits. As mentioned above, some passive cooling of the spectrometers is obtained by radiation to the wall of the rover.

During installation a concern arose about potential contamination of the UV optics by outgassing of other spacecraft components during cruise. Additional heaters were added to the spectrometers, CCD covers, and demultiplexer to maintain these parts above the ambient temperature during cruise. Figure 16 shows the position of the BU relative to the rover wall, and shows the new heaters on the top of the spectrometers.

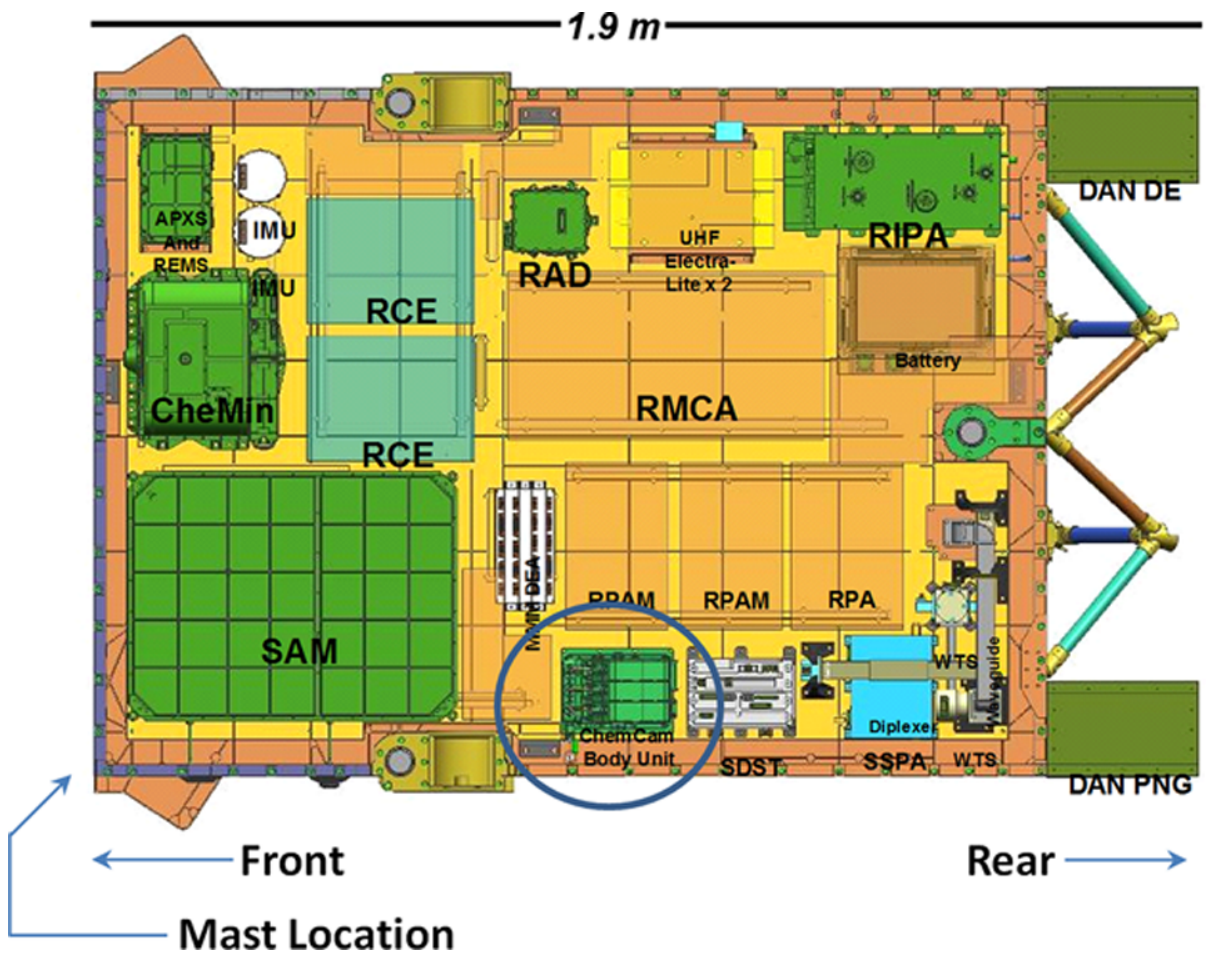

Fig. 15 ChemCam body unit location in the rover, looking from the bottom up. Science instruments shown include (counterclockwise from top left) APXS (Alpha Particle X-ray Spectrometer), CheMin (X-ray diffraction experiment), SAM (Sample Analysis on Mars), DAN (Dynamic Albedo of Neutrons, with separate sections for the pulsed neutron generator and the detection electronics), and RAD (Radiation Assessment Device). Other rover assemblies shown include the redundant RCE (rover compute element) boxes, RIPA (rover integrated pump assembly), RMCA (rover motor control assembly), RPA (rover power assembly), RPAM (rover power and analog module), SDST (small deep space transponder), and SSPA (solid state power amplifier) 
Fig. 16 Close-out image showing the darkened rover wall on the left and the spectrometers on the right. Cruise

contamination heaters are in the foreground (mounted with epoxy), while the fiber bundles are attached to the spectrometers in the upper right. The mast-to-body optical fiber is seen as a black cable and connector at the center of the image where it attaches to the demultiplexer, and the TEC is in the lower center. The three thermal conduits are visible as the reflective cables seen in the lower center of the image

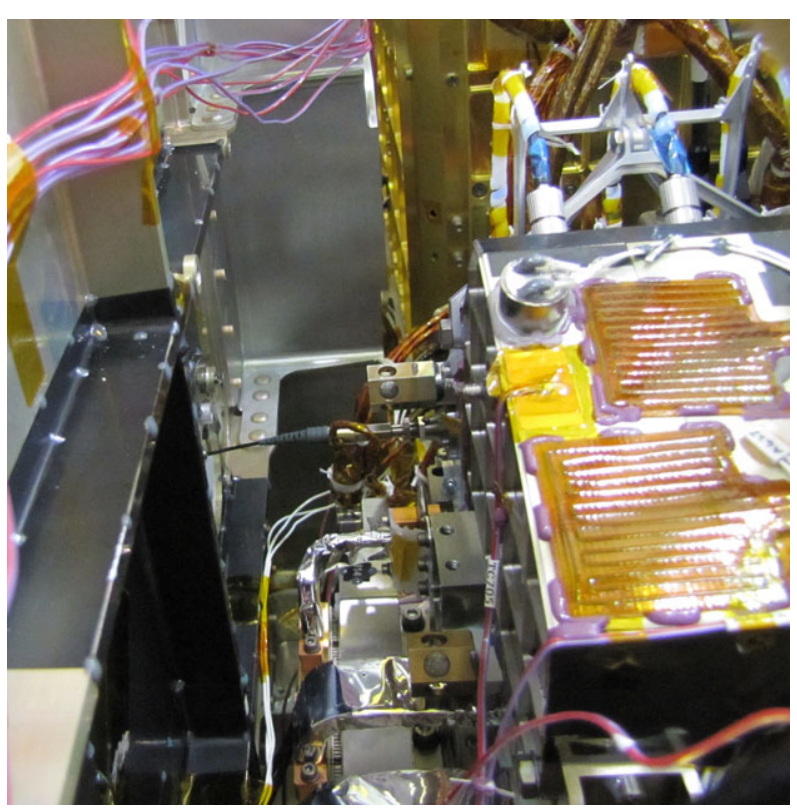

\subsection{Mast-to-Body Fiber}

The fiber optic cable (FOC) traverses the distance between the mast and body units, mostly along the mast, but also along the deck of the rover. From the connector on the rear of the MU the fiber makes a U-turn to bring it to the opposite side of the remote warm electronics box (RWEB) where it enters the elevation twist cap (Fig. 17) and from there goes into the azimuthal twist cap. As discussed in Sect. 3.1, the twist caps are designed to pass the optical and electrical cables around the respective gimbals without stressing the cables. After traversing the twist caps the fiber proceeds down the exterior of the mast to the base, where one more small twist cap routes the fiber around the bottom joint (Fig. 17), which is used for a one-time deployment of the mast after landing on Mars. From here the fiber runs across the deck to a location near the BU, where it enters the body of the rover and connects to the BU (Fig. 16).

\subsection{Calibration Target Assembly}

The calibration target assembly is located on the back of the rover at an angle of $37.9^{\circ}$ from vertical, and facing the MU, as shown in Fig. 18. The RSM points $28.5^{\circ}$ below horizontal to acquire the centerline of the target assembly, the normal of which is pointing $9.4^{\circ}$ above the line of sight to ChemCam. The calibration target assembly is $1.56 \mathrm{~m}$ from the Schmidt plate of the MU (Maurice et al. 2012). Each round LIBS target provides an angular cross section of $11.7 \mathrm{mrad}$ at this distance. An image of the Ti plate is shown in Fig. 19 as it is seen by the RMI. A single laser pit is clearly visible near the center of the image. 
Fig. 17 View of the rover mast showing the Remote Warm Electronics Box (RWEB) housing the ChemCam mast unit, cable twist caps, and mast deployment joint. The mast is approximately $1 \mathrm{~m}$ in height; overall the ChemCam aperture is $2 \mathrm{~m}$ above the Mars surface
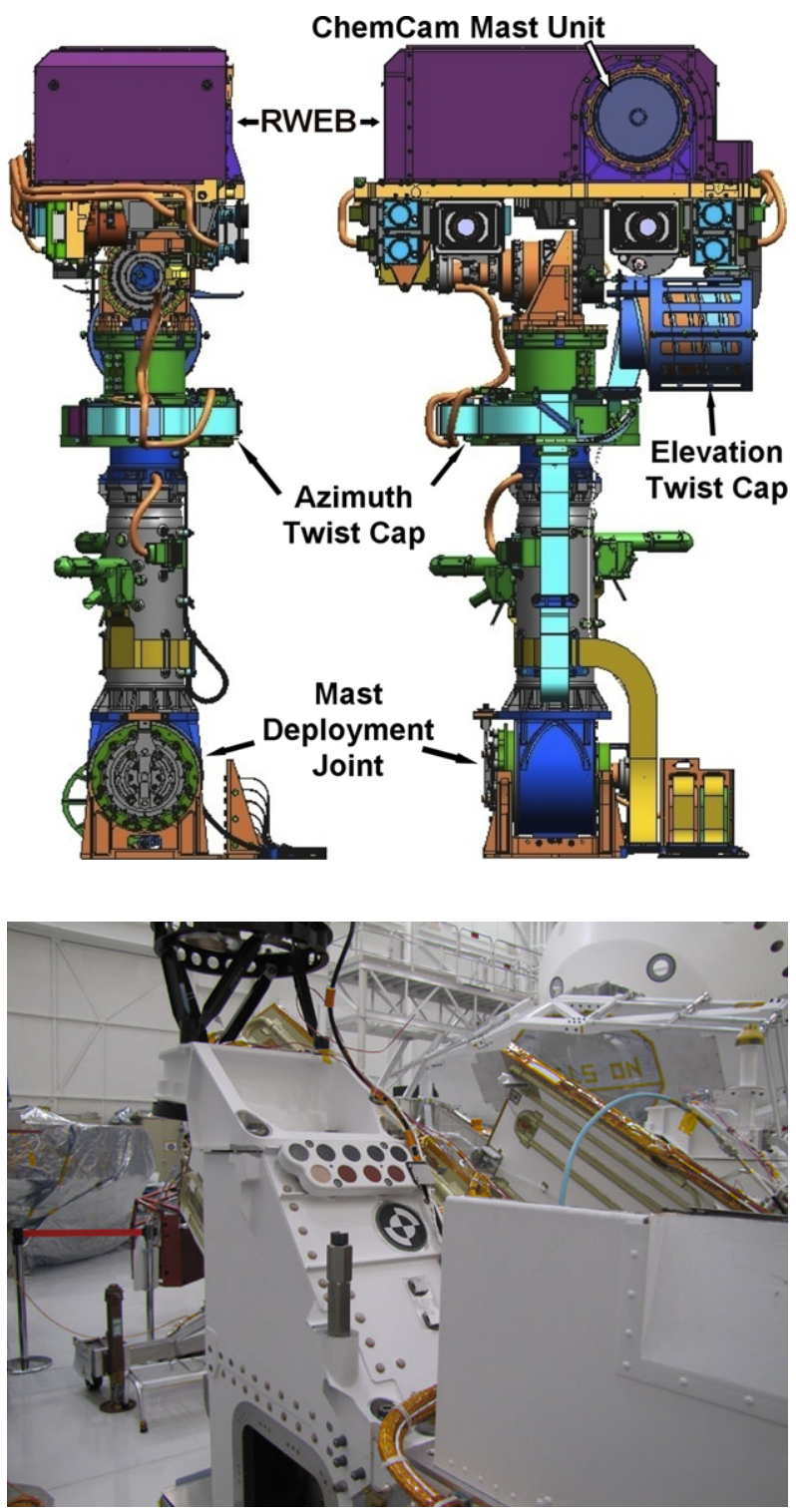

Fig. 18 ChemCam calibration target (CCCT) assembly in its location at the rear of the rover, directly behind the mast

\section{Operations}

\subsection{Basic Concepts and Commands}

Figure 20 shows a flow diagram for a typical single ChemCam observation. A science target is selected, usually based on images downlinked from a previous sol. The team envisions also using autonomous targeting in some cases later in the mission. This could be done for random soil sampling, for example during a long drive, by blindly targeting a point $\sim 1 \mathrm{~m}$ in front of the rover. Autonomous targeting may also be done using a JPL software package such as AEGIS (Estlin et al. 2010), a version of which has been used on the Mars Exploration 
Fig. 19 RMI image of the Ti plate (25 $\mathrm{mm}$ square) on the ChemCam rover calibration target (CCCT) assembly, with a single bright laser pit near the center. The image was taken during pre-flight rover testing

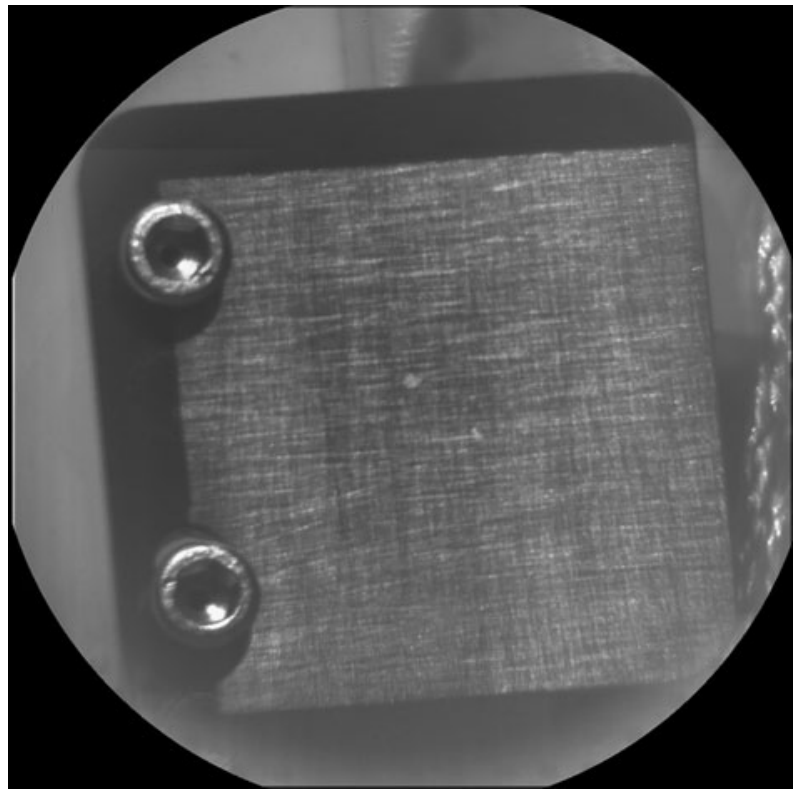

Fig. 20 Flow chart for a typical single ChemCam observation, includes an RMI image before and after a LIBS observation. A single observation, including LIBS spectra and RMI images of one location takes about six minutes from the time the lasers are warm until the time the lasers are cooled

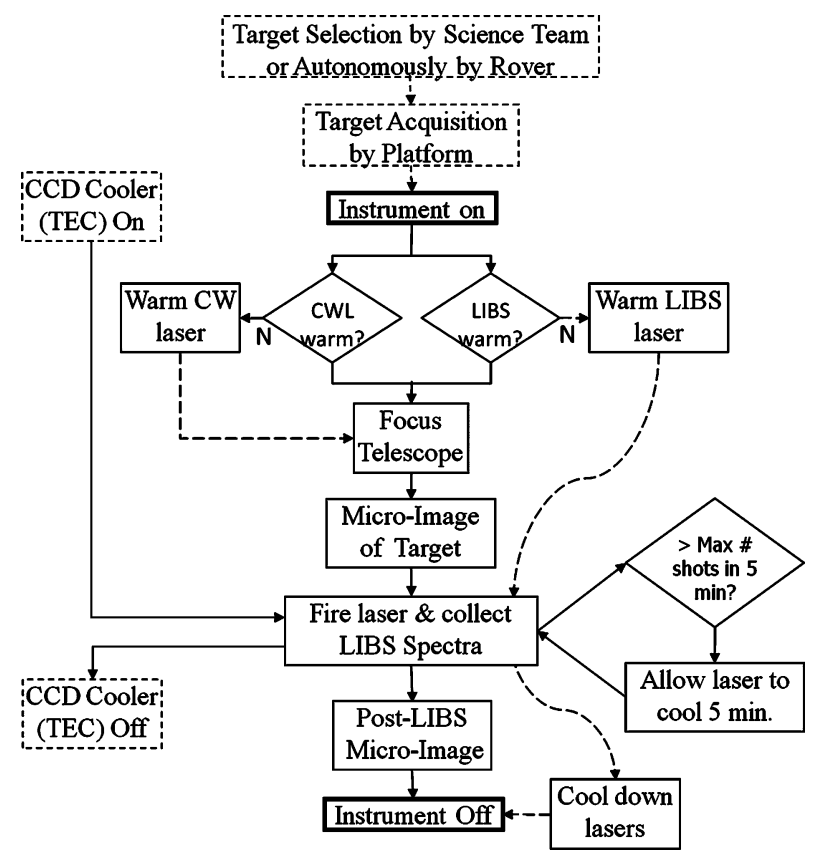

Rovers (MER) to identify and prioritize rocks in the field of view of the cameras. The mast is used to point ChemCam at the desired target and the ChemCam instrument is powered on. Note that the mast coordinates are commanded as part of the ChemCam activity sequence. In order to perform auto-focusing, the continuous-wave laser (CWL) used in this process must be warmed to $10{ }^{\circ} \mathrm{C}$ at a rate of $5{ }^{\circ} \mathrm{C}$ /minute (Maurice et al. 2012). Assuming that 
a LIBS observation is to be done, as is shown in the Fig. 20 flow, the LIBS laser may require heating to the lower end of its operating range of -20 to $+20{ }^{\circ} \mathrm{C}$, also at a rate of $5{ }^{\circ} \mathrm{C} /$ minute. As soon as the CWL is at its operating temperature the telescope can be focused. After focusing, ChemCam is ready to take an RMI image of the target. In case the CWL fails, backup methods have been considered for determining the optimum focus of the LIBS laser (Barkley 2008). More details of the MU operations, including focusing, taking images, and firing the laser are given in the companion paper (Maurice et al. 2012).

Once the RMI image is transferred to the rover, ChemCam fires the laser and collects spectra. Most LIBS observations consist of a number of laser pulses (e.g., 20) and corresponding spectra which can be averaged together either on board the rover or on the ground to provide better statistics than obtained from a single-laser-pulse spectrum. If, after a number of sols of LIBS observations, the team determines that the dust layer on rocks takes no more than a certain number of laser pulses to remove, and the dust has already been fully characterized, that number of initial laser pulses can be ignored, with spectral collection starting subsequent to these pulses. Additionally, depth profiles of a thousand or more laser pulses may be done to study possible rock coatings. If needed, a wait state is used to allow the laser to cool. After the LIBS spectra are collected and transferred to the RCE, a post-LIBS image is taken. A sub-frame showing only the LIBS observation point can be downlinked, saving bandwidth over a full image. A final step is to control the cooling rate of the CWL and LIBS lasers, using the same thermal ramp rate given above. The focus stage is parked at a sun-safe position, designed to protect the instrument when the mast points temporarily at the Sun, and the instrument is then turned off.

The thermo-electric cooler (TEC) can be turned on and off independently of ChemCam, allowing it to be started before the instrument itself if this provides the most efficient operation.

Commands to ChemCam go through several interfaces before being implemented on the instrument. Tactical operations of the rover are planned through a mission graphic user interface called MSLICE, which contains ChemCam "activities", with adjustable parameters. Once these activities are approved for operation, they are translated into spacecraft commands (s-cmds), which are uplinked to the rover and unpacked. ChemCam-related s-cmds can control the mast, the TEC, and the instrument itself. Those controlling the instrument are translated into "i-cmds" and are sent to the instrument DPU at the proper time where they are unpacked and acted upon. Instructions for the mast unit ("m-cmds") are sent from the BU to the MU, for example, for firing the laser, taking an RMI image, or focusing.

Figure 21 shows a high-level state diagram for the flight software. Booting is a transitory process. Once on, the instrument is either idle, in which case it is available for commanding, or it is "busy" while processing a command. The instrument can be removed from the busy state via the abort command, which does not affect the status of the heaters. The reset command will, however, turn the heaters off. The Power Off or Safe commands return the instrument to the Off state. ChemCam has no non-volatile data memory, so all data must be sent to the RCE before powering off. Due to the relatively small amount of data memory resident in the instrument, the data are normally transferred immediately after it is obtained.

The instrument's major commands are CCAM_RMI_OBS for imaging, CCAM_PASV_ SPECTRAL_OBS for taking passive spectra without using the LIBS laser, and CCAM_ACTV_SPECTRAL_OBS for taking active LIBS spectra. A separate command, CCAM_CAL_TARGET_OBS, is used for targeting the on-board calibration targets, as these require special attention for pointing the mast to avoid accidentally shooting at the rover. The repertoire of commands includes others for specific purposes, particularly for troubleshooting and for obtaining state-of-health data. 
Fig. 21 High level state diagram of rover flight software controlling ChemCam. Sequences of ChemCam commands which also involve articulating the rover's remote sensing mast (RSM) are listed above the blue circle labeled "Pointing RSM"

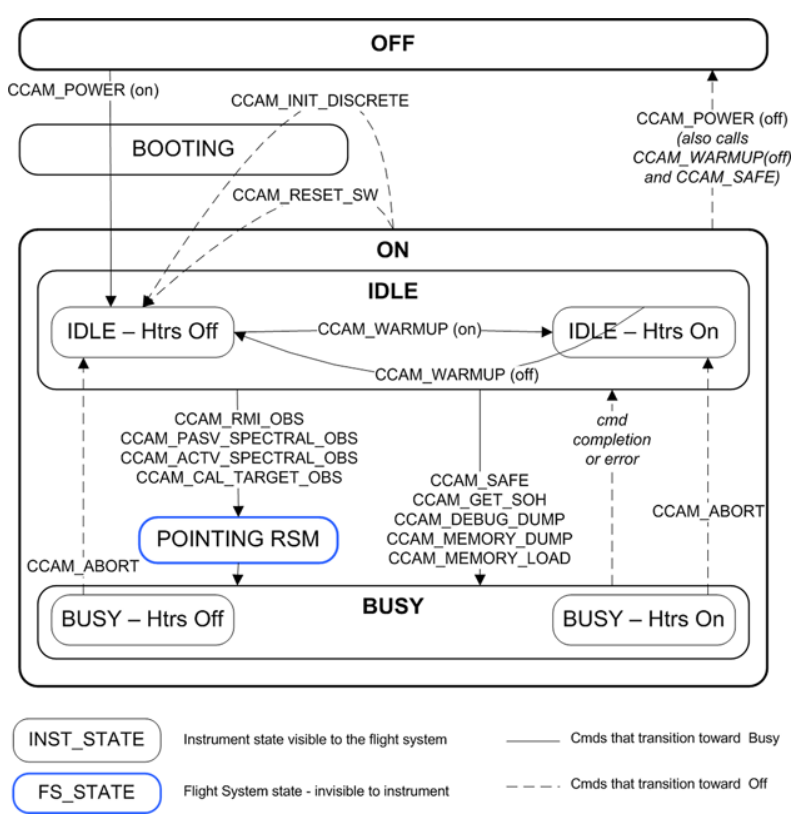

\subsection{Parameters and Operation Modes}

While a typical observation example was given above, operation of ChemCam is very flexible: RMI images can be taken without LIBS or vice versa. The telescope can be focused manually or automatically, and the RMI can use manual exposure settings or auto exposure (Maurice et al. 2012). The MSLICE activities facilitate the use of LIBS line scans or 2D rasters in which multiple LIBS observations are made in a 1D or 2D pattern. These can be done quite rapidly if there is no need to re-focus in between points.

The LIBS operation itself is also flexible. LIBS spectra can be taken at a rate anywhere between 1 and $10 \mathrm{~Hz}$, though the default rate is $3 \mathrm{~Hz}$. Up to 150 spectra can be captured at this rate before pausing to transfer the data. Each spectrum consists of 6444 channels: 2048 active channels and 100 masked channels in each spectrometer. The spectral data are digitized as fourteen bits per channel which is packed into two words (16 bits), with the result that each spectrum is $12.9 \mathrm{kB}$. Downlinking each spectrum over many laser pulses results in a large data volume and so there are a number of options to reduce it. The simplest is to sum a number of spectra for one observation point. The instrument software accommodates on-instrument summing of different numbers of spectra during a single observation. For example, ChemCam can bin twenty spectra into a single sum, or two sums of ten each, four sums of five each, ten sums of two each, or even sums in between these (but with an incomplete number of sums in the final bin, in this case). Each sum is packed as 32 bits to avoid overflow. As mentioned earlier, ChemCam also has a "shots to ignore" parameter which disables the collection of spectra over the specified number of pulses at the beginning of a burst of laser pulses to avoid dust contamination.

Although summing reduces the data volume, it does so at the cost of potentially missing compositional and statistical variations in the data. Another data treatment pathway is available in which all of the spectra are passed to the RCE which processes the data to provide a mean, median, first and third quartile, and standard deviation. The latter parameter can inform the team about the quality of the data and the heterogeneity of the sample, which 


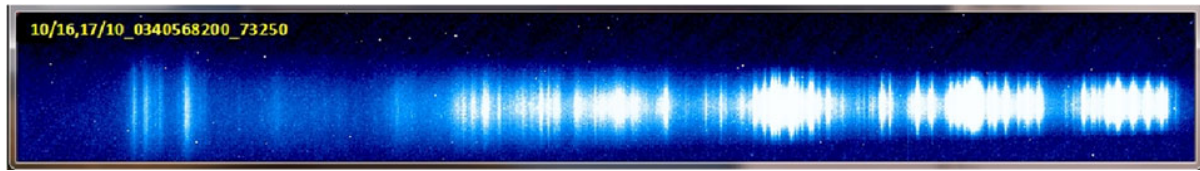

Fig. 22 Two-dimensional image of LIBS emission lines from a titanium target in air as captured on the UV CCD. The exposure was $2 \mathrm{~s}$ with the laser at $10 \mathrm{~Hz}$, recording from row 50 to row 306, during testing at JPL. The image is inverted, with row 50 at the top. The shortest wavelength is at the left

may indicate the presence of dust or weathering alteration in the spectral data. The quartiles can be omitted to reduce data volume, but they are useful to detect the presence of outliers among the individual spectra. This "statistics" data path should be available after the first software upload after landing.

While ChemCam normally uses short exposures to capture each plasma spectrum separately, the CCDs can also capture multiple plasmas during a single long (i.e. hundreds of $\mathrm{ms}$ to several second) exposure. This long-exposure mode is mostly used for diagnostic testing, as when it is combined with the 2D spectral imaging parameter it displays the position of the light on the detectors, as shown in Fig. 22, allowing a check for position drift in the spatial as well as spectral directions. To operate in this mode the laser repetition rate is increased to $10 \mathrm{~Hz}$ to maximize the ratio of signal to background light. Using the 2D parameter, data are collected from only one spectrometer at a time, and only half of the CCD at that, due to memory limitations on the spectrometer board. Rows 1-256 can be collected in one exposure and 257-512 with the next command for each spectrometer, using six commands to collect data from every pixel of all three spectrometers.

Under the proper conditions the long-exposure mode will produce spectra with higher signal to noise than multiple individual exposures. This may be the case under low ambient light conditions and low plasma signal, i.e., at long distances, when the CCDs are cold and ambient lighting is minimal. Because the summing is on-chip, the total counts collected in the sum of the spectra must be below the fourteen bit limit per channel.

Instrument power was measured under various conditions to understand its operation even at voltage and temperature extremes. ChemCam uses at minimum 11.8 W (BU: 7.2 W, MU: $4.6 \mathrm{~W}$ ) while idling, and up to a maximum of $64.7 \mathrm{~W}$ (BU: $20.8 \mathrm{~W}$, MU: $43.9 \mathrm{~W}$ ) while firing the laser at $10 \mathrm{~Hz}$, though this state lasts a very few seconds. The instrument uses only half this power if firing the laser at a lower rate, such as $1 \mathrm{~Hz}$. In addition to the power drawn by the instrument itself, the TEC, which is operated independently, draws a constant $13.5 \mathrm{~W}$. The energy and power discussed in this section does not include that required to position the mast. Potentially the largest power draw is the energy required to pre-heat the mast motors if analyses are desired before the mast is warmed naturally by the sunlight. This can consume up to $\sim 100 \mathrm{~W}$-hr depending on the duration of heating.

Table 6 presents the durations and energy required to perform various ChemCam activities, following the flow introduced in Fig. 20. Unless otherwise noted, each case assumes a nominal thermal condition. Entries 3, 4, and 5 present a single LIBS observation consisting of 75 laser pulses in one location, preceded and followed by RMI images. However, a relatively large proportion of the energy is consumed in warming the laser, and if the TEC is used, in cooling the detectors, so efficiencies are gained by making additional analyses once ChemCam is ready for operation. The lowest additional expenditure occurs when carrying out a line scan, for example, five observation points in close proximity such that re-focusing is not needed. Line 6 in Table 6 shows that the additional four observation points require only an additional $\sim 3$ minutes and $\sim 6 \mathrm{~W}$-hr. The additional observation points can be used 
Table 6 Energy and durations required for ChemCam analyses. Times include target acquisitions

\begin{tabular}{llllll}
\hline Activity & \multicolumn{2}{l}{ Duration (hr:min:sec) } & & \multicolumn{2}{c}{ Energy (W-hr) } \\
\cline { 5 - 6 } & No TEC & With TEC & & No TEC & With TEC \\
\hline 1. RMI image (autofocus + autoexposure) & $0: 05: 40$ & N/A & 2.3 & N/A \\
2. RMI image + passive spectrum & $0: 08: 32$ & $0: 23: 02$ & 5.3 & 9.4 \\
3. RMI pre-image + LIBS + post-image (warm) & $0: 18: 22$ & $0: 31: 22$ & 8.7 & 11.6 \\
4. RMI pre-image + LIBS + post-image (nominal) & $0: 23: 22$ & $0: 31: 22$ & 12.2 & 13.8 \\
5. RMI pre-image + LIBS + post-image (coldest) & $0: 31.22$ & $0: 31.22$ & 17.8 & 21.2 \\
6. RMI + LIBS line scan (5 points) & $0: 25: 26$ & $0: 34: 36$ & 18.0 & 19.6 \\
7. RMI pre-image + Depth Profile ${ }^{\mathrm{c}}+$ post-image & $0: 36: 34$ & $0: 43: 34$ & 23.3 & 24.8 \\
8. 7 targets (RMI + LIBS as in \#4) & $1: 08: 34$ & $1: 15: 34$ & 38.7 & 40.3 \\
9. 20 targets (RMI + LIBS as in \#4) & $2: 44: 20$ & $2: 51: 20$ & 94.7 & 96.2 \\
\hline
\end{tabular}

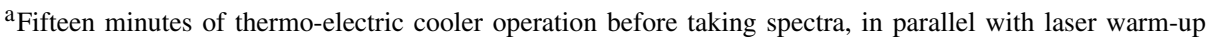
when suitable

${ }^{\mathrm{b}}$ No re-focusing in between, RMI image after completion

${ }^{\mathrm{c}}$ Depth profile in this case consists of 900 laser pulses in one location and corresponding spectra

to constrain the composition of the rock or soil by acquiring data from additional locations. A line scan could also be used to locate a very small feature not likely to be acquired with a single attempt of the RSM. In the same way, 2D rasters can also be used to target small objects. Lines 8 and 9 in Table 6 present durations and energy for analyzing numerous individual targets as might be done on a reconnaissance sol. Each additional ChemCam target requires an incremental duration of approximately 7.5 minutes and $4.4 \mathrm{~W}$-hr. Line 7 presents the duration and energy for a depth profile of 900 laser pulses, requiring approximately half an hour and $24 \mathrm{~W}-\mathrm{hr}$. All of the values presented here are believed to be conservative, so that expected resource usage may be slightly lower in actual operation.

\subsection{Data Processing and Products}

RMI images are processed into reduced data record (RDR) images automatically and immediately upon receipt from the Deep Space Network. An algorithm is applied to automatically correct for dark noise, ghost images, smear, and vignetting (Maurice et al. 2012). The RDR image is available as a quick-look product which provides the context (location, before or after LIBS, etc.). More advanced data products, such as difference images after-before LIBS, mosaics, z-stack, or entropy products may be produced by the team on an occasional basis.

LIBS data are processed into quick-look products immediately and automatically upon receipt. All quick-look products are produced in jpeg format. Spectra for a given observation point are averaged (if separate spectra are downlinked from each laser pulse), the non-laser background is subtracted, the spectra are treated by a de-noise and sanity check algorithm, and the electron emission (bremsstrahlung) background is removed. Resulting spectra are displayed against preliminary wavelengths with one panel for each spectral range. The positions of major peaks typically encountered in geological samples are indicated. Each spectral range is normalized separately for ideal viewing, but the maximum counts and the sum of all counts for each spectral range are also given. Additional information about the sample location, number of laser pulses, laser energy, and distance is also given. The LIBS spectral quick-look plots allow rapid identification of elements present and, for people familiar with 
relative emission line cross sections, give a qualitative idea of the relative abundances of the different elements present.

A second LIBS quick-look product is available in the form of stack plots for analyses in which multiple spectra are downlinked, including depth profiles. In these plots the intensities are given as a function of wavelength for each successive spectrum that is downlinked, with the intensity indicated by color, and the spectrum \# increasing along the y-axis. These plots allow a quick determination of the vertical heterogeneity and trends within the observation point.

Several RDR LIBS products are planned. The first two, somewhat like the quick-look spectra, are simple spectra that have been fully calibrated for wavelength and have the nonlaser background subtracted and the bremsstrahlung continuum removed; unlike the quicklook product, the RDR product is in comma-separated value (csv) form. One version folds in the instrument response and the other version gives the spectral results in digital numbers. The remaining RDR formats generally require comparison with standards. The team envisions two products which classify and compare the samples without giving elemental composition predictions. One such product uses independent component analysis (ICA; e.g., Comon 1992) coupled with a clustering analysis, outputting the results as a csv file that gives the Euclidean and Mahalanobis distances between the new observation and the closest known sample (Forni et al. 2009). Another product generates a jpeg image of a Sammon's map, a non-linear two dimensional representation which uses an iterative approach to find the global minimum of the inter-point distance in the original multidimensional space by following a steepest descent procedure (Sammon 1969). Sammon's maps of ChemCam calibration data are displayed and discussed in Lasue et al. (2011).

A final RDR product is quantitative elemental compositions and uncertainties which are predicted for the major elements and selected minor and trace elements, likely to routinely include $\mathrm{H}, \mathrm{Li}, \mathrm{O}, \mathrm{Na}, \mathrm{Mg}, \mathrm{Al}, \mathrm{Si}, \mathrm{K}, \mathrm{Ca}, \mathrm{Ti}, \mathrm{Mn}, \mathrm{Fe}, \mathrm{Rb}, \mathrm{Sr}$, and $\mathrm{Ba}$. In addition, $\mathrm{C}, \mathrm{N}$, $\mathrm{F}, \mathrm{P}, \mathrm{Cl}$, and $\mathrm{S}$ may be included if above $\sim 5 \mathrm{wt} \%$, and $\mathrm{Cr}, \mathrm{Ni}, \mathrm{Zn}, \mathrm{Zr}, \mathrm{Cu}$, and $\mathrm{Pb}$ may be reported if more than $\sim 300 \mathrm{ppm}$. These are predicted by comparison with training sets of standards using partial least squares (PLS) regression of principal components, a technique which appears best suited to deal with the chemical matrix effects that complicate quantitative analyses of geologic targets by LIBS (Clegg et al. 2009; Tucker et al. 2010; Dyar et al. 2011; Anderson et al. 2011). More details of the ChemCam LIBS training set, data reduction methods, accuracies and detection limits will be given in a forthcoming paper. Cross-calibration with APXS (Gellert et al. 2012), CheMin (Blake et al. 2012), and SAM (Mahaffy et al. 2012) is also expected to be used on Mars.

\section{Performance Testing}

Table 7 gives a summary of the LIBS integrated performance tests that were undertaken prior to delivery to the rover. They were started in October, 2008, in preparation for the 2009 launch. However, changes were made to both the BU and MU after the launch delay was announced. For the MU a resistor was added, which improved the laser performance, particularly at cold temperatures. This was done to compensate one of the resistors on the Poeckel cell circuit which had a higher resistance than expected at cold temperatures, resulting in poor performance at moderate to high laser repetition rates, particularly when fired immediately after enabling the laser. The BU had its demultiplexer and detectors replaced and the TEC installed, as mentioned above. Because these changes affected the performance quite significantly, only the more-recent performance testing in 2010 and 2011 is discussed here. 


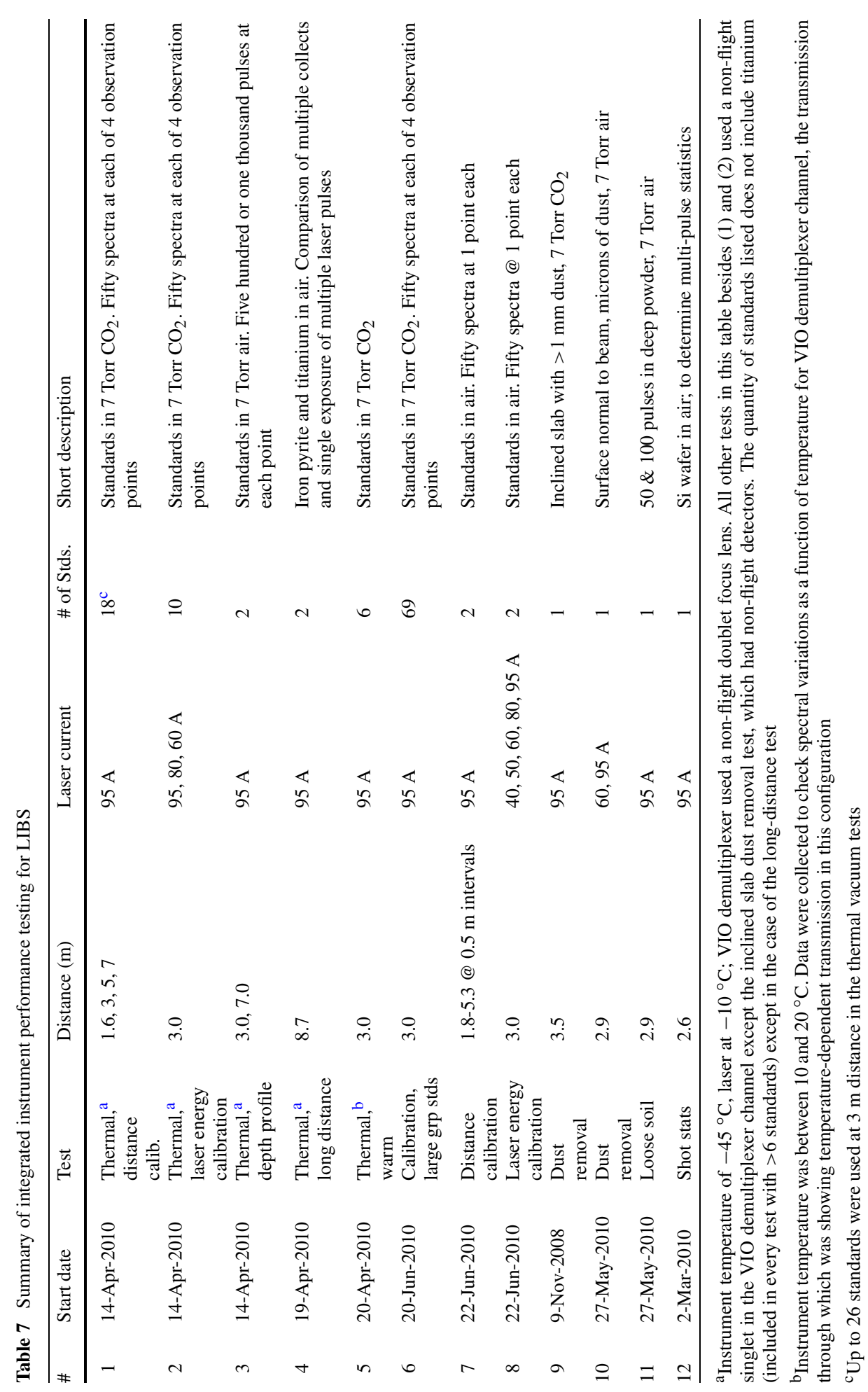


Comparison of different pre-delivery performance test results (Table 7) is complicated by changes to the body unit optical path during the course of these tests. As alluded to above, for the thermal test the demultiplexer VIO channel used a lens that was later discovered to have temperature-dependent transmission at the low end of the wavelength range (see Sect. 3.2). Between thermal testing and the final calibration this lens was replaced with a second lens which turned out to be inferior to the original LANL lens. So after final calibration the lens was replaced again prior to delivery. As a result of these changes, inter-comparison between different test data sets requires correction. Spectra have been compared from different times during the instrument characterization and, after correction for optical response, the results appear very similar.

The LIBS tests listed in Table 7 can be broken down into two classes: those which used a significant number of standards (e.g., more than five) for the purpose of cross-calibration between standards, and those using a small number of standards (one or two) to check some aspect of the observation other than elemental composition accuracy. These will be discussed in separate sections below. All tests made at low pressure involved manual focus, as reflections from the window of the sample chamber interfered with the autofocus routine.

Testing of the RMI at the integrated instrument level was limited to ensuring the proper transfer of the images and to determining the proper implementation of the autofocus and offsets (Maurice et al. 2012).

\subsection{Instrument Optical Response}

The instrument optical response was measured in all of the configurations for which calibration data were taken, including during thermal vacuum testing, at room temperature, and after integration into the rover. The instrument response in the first two cases had to include other optical elements inherent in the set-up, including the window for the Mars sample chamber for the room-temperature calibrations. For the thermal vacuum tests the instrument thermal chamber window and a turning mirror had to be included in addition to the sample chamber window. The arrangement is shown in Fig. 23. The transmission of the sample chamber was measured separately. The rover transmission tests at JPL included the RWEB window.

The optical response of the VIO and VNIR spectrometers were determined using a calibrated Labsphere integrating sphere and lamp assembly. This standard provided spatially uniform radiance, generally on the $2 \%$ or better level, across its $\sim 100 \mathrm{~mm}$ aperture and over angles up to $9.5^{\circ}$. For these measurements the ChemCam telescope was focused on the

Fig. 23 Experimental set-up for ChemCam LIBS thermal vacuum tests. The instrument is in the large white thermal chamber to the right, a turning mirror is in the foreground, and the sample chamber is to the left, providing an instrument-to-sample distance of $1.5 \mathrm{~m}$. For tests with more distant sample positions the sample chamber was moved back, into the adjoining room

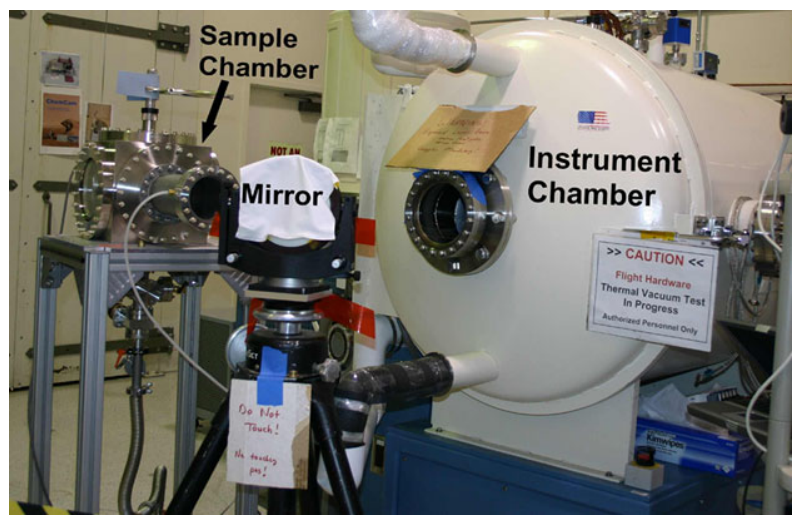


aperture plane or a few centimeters in front of it. Uniformity of the back surface sufficient for the expanding angle through focus was verified with both lateral and longitudinal displacements of the sphere, and no changes in sensor output beyond sensor noise levels were noted. Given the large source area and ChemCam's small field of view, constant radiance is provided for all relevant viewing distances for ChemCam. The system response in digital numbers $(D N)$ can be written as:

$$
D N=L \cdot A \cdot \Omega \cdot \Delta \lambda \cdot \Delta t \cdot t_{0} \cdot \sigma+D N_{0}
$$

where $L$ is the source radiance in photons $/ \mathrm{second} / \mathrm{cm}^{2} / \mathrm{sr} / \mu \mathrm{m}$ wavelength, $A$ is the source area imaged, in $\mathrm{cm}^{2}, \Omega$ is the solid viewing angle in $\mathrm{sr}, \Delta \lambda$ is the detector wavelength bin width in $\mu \mathrm{m}, \Delta t$ is the integration time in seconds, $t_{0}$ is the system throughput multiplied by the detector quantum efficiency, in electrons per photon, $\sigma$ is the amplified conversion gain in $D N / e l e c t r o n$, and $D N_{0}$ is the offset due to stray light and electrical offsets from zero DN. The last term can be removed by subtracting a spectrum obtained with the radiance source turned off. All measurements are made with the room lights off. The equation can then be solved for the product of $t_{0} \cdot \sigma$, giving the optical response for each spectral bin in photons per $D N$.

The UV spectral range had to be calibrated in a slightly different way due to the lack of an integrating sphere standard irradiance source in that wavelength range. A fiber-receptacle irradiance standard was used to characterize the UV range from a flight spare FOC through the spectrometer, and the telescope transmission was measured separately:

$$
D N=I_{\lambda} \cdot A \cdot \Delta \lambda \cdot \Delta \cdot t \cdot t_{\text {tel }} \cdot t_{\text {back }} \cdot \sigma+D N_{0}
$$

where $I_{\lambda}$ is the reference source irradiance in photons $/$ second $/ \mathrm{cm}^{2} / \mu \mathrm{m}$ wavelength, $A$ is the area of the fiber tip being illuminated, in $\mathrm{cm}^{2}, t_{\text {tel }}$ is the telescope throughput, and $t_{\text {back }}$ is the back-end throughput multiplied by detector quantum efficiency. The product $t_{\text {tel }} \cdot t_{\text {back }}$ is equivalent to $t_{0}$ above. The telescope throughput $\mathrm{t}_{\text {tel }}$ was obtained via measurements where the FOC directly viewed a small integrating sphere illuminated by a large UV source. This measurement was subsequently compared with the spectrometer output when the fiber was illuminated by the telescope image of the sphere radiance.

As it was impossible to de-mate the FOC from the telescope after ChemCam was integrated on the rover, UV range calibration on the ATLO floor employed a fiber-receptacle irradiance standard and the telescope was aimed at the fiber tip. Given the very narrow $<1 \mathrm{mrad}$ field of view of the spectrometers, alignment was a significant challenge even with taking multiple images with the RMI to align the fiber tip. The resulting measurement showed the correct spectral shape but gave weaker response due to misalignment. Comparison of the UV ranges of the LIBS spectra suggested that no unexpected changes had occurred during integration of the instrument.

Figure 24 shows the optical response for all three spectral ranges as obtained for the final rover configuration. The instrument response is a composite of the transmission of all different parts of the optical path along with the quantum efficiency curves and gains of the detector circuits. The spectrometers have some vignetting at the edges of the spectral ranges, and the dielectric mirrors contribute a few minor absorption features across the spectrum. The demultiplexer dichroic mirrors also contribute to a reduction of transmission near the gaps between the three spectral ranges. The FOC between the MU and BU reduces the transmission in the far UV and has a small $\mathrm{OH}$ absorption feature around $725 \mathrm{~nm}$ (cf. Fig. 4). In the MU the laser dichroic cuts out at the third harmonic of the laser wavelength of $1067 \mathrm{~nm}$, which defines the gap between the UV and VIO spectral ranges (Maurice et al. 2012). The VNIR useful range is limited at the lower end by the transmission cut-off of 
Fig. 24 ChemCam end-to-end optical response in terms of digital number (counts received) per photon over the UV, violet, and VNIR spectral ranges
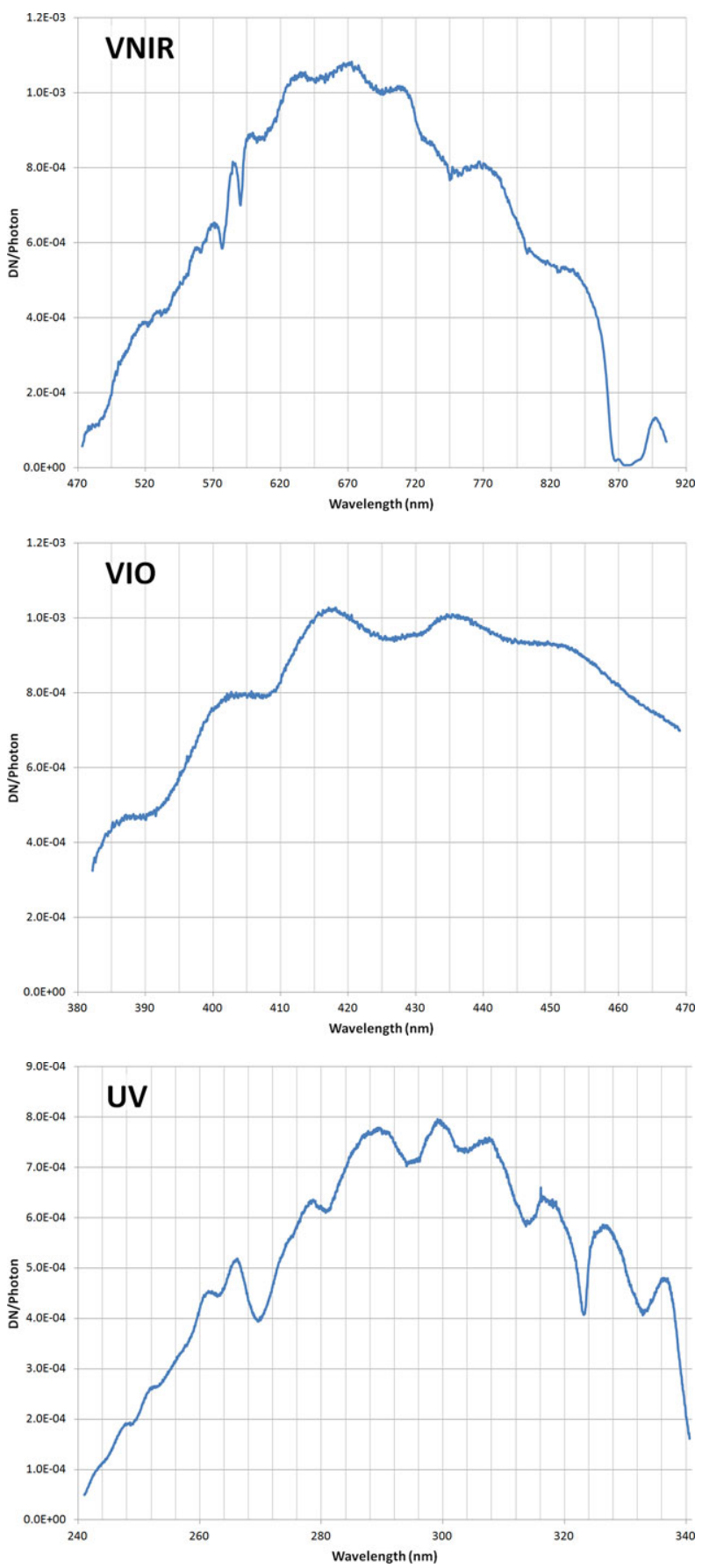
the VIO-range dichroic mirror in the demultiplexer, so the response reaches a value of 1e-4 $\mathrm{DN} /$ photon around $480 \mathrm{~nm}$. Above $855 \mathrm{~nm}$ the signal is limited due to optical attenuation by the spectrometer mirrors, which were only specified to the manufacturer up to $800 \mathrm{~nm}$. The result is poor throughput from $855-890 \mathrm{~nm}$. However, there is a window between 890 and $906.5 \mathrm{~nm}$ where the response is better than $1 \mathrm{e}-4 \mathrm{DN} /$ photon, and this window might be used for passive spectroscopy.

\subsection{LIBS Spectra, Elemental Abundance Accuracy, and Sample Classification}

Figure 25 shows a LIBS spectrum of basalt standard BT-2 (Broken Tank, NM), with a composition of 49 wt. $\% \mathrm{SiO}_{2}, 17 \% \mathrm{Al}_{2} \mathrm{O}_{3}, 11 \% \mathrm{Fe}_{2} \mathrm{O}_{3}$ (total), $6.4 \% \mathrm{MgO}, 7.9 \% \mathrm{CaO}, 4.5 \%$ $\mathrm{Na}_{2} \mathrm{O}, 1.3 \% \mathrm{~K}_{2} \mathrm{O}, 1.5 \% \mathrm{TiO}_{2}, 0.44 \% \mathrm{P}_{2} \mathrm{O}_{5}, 0.15 \% \mathrm{MnO}, 480 \mathrm{ppm} \mathrm{Ba}, 120 \mathrm{ppm} \mathrm{Cr}$, 83 ppm Ni, 50 ppm Cu, 560 ppm Sr, 195 ppm V (P. King, personal communication, 2010). The spectrum was acquired during the thermal-vacuum testing at $3 \mathrm{~m}$ (Tests \#1-2 in Table 7) with the laser current at $95 \mathrm{~A}$. Of fifty individual spectra, the first five were removed, as they show evidence of water absorption and surface contamination. The remaining spectra were averaged and the non-laser background was subtracted. The continuum was not removed and can be seen as the low baseline below the peaks, particularly in the VNIR region. The major peaks are identified, based on the Mars emission line database by Cousin et al. (2012). Emission lines from $\mathrm{Ba}, \mathrm{Sr}$, and $\mathrm{Cr}$ can be seen in spite of their low abundances. A much higher density of emission lines, particularly from transition elements, is apparent in the UV and VIO ranges when considering the change in scale for the VNIR plot.

The LIBS signal intensity decreases with increasing distance approximately as the reciprocal of the distance cubed (Table 7, Tests \#1 and 7) because of the combination of capturing a smaller solid angle of the returned light $\left(r^{-2}\right)$ and lower power density from the interrogating laser resulting from poorer focusing at greater distance. At $7 \mathrm{~m}$ the strongest emission lines are nearly always below 1000 counts/pulse, with weaker lines approaching the detection limit, making this distance effectively the farthest reach for quantitative results. The maximum distance of operation depends quite strongly on the coupling of the laser to the sample, which can vary by over two orders of magnitude for geological samples (Anderson et al. 2011). Some samples did not couple well even at $6 \mathrm{~m}$ in laboratory testing, while a pyrite sample gave a spectrum at nearly $9 \mathrm{~m}$ (Table 7, Test \#4). In general, materials rich in transition metals are strong couplers, but a lack of transition metals does not necessarily result in weak coupling. Martian samples typically contain relatively high Fe contents compared to an average terrestrial rock (Clark et al. 1976), so samples are expected to couple relatively well on Mars. On the other hand, closer than $3 \mathrm{~m}$ the signal increases significantly so that strong emission lines can easily saturate unless the laser intensity is lowered.

Tests \#1 and 6 in Table 7 were performed with a number of different samples and these results were used to determine the accuracy and precision of ChemCam quantitative analyses at various distances using PLS regression, which has been used by the ChemCam team and collaborators to determine semi-quantitative elemental compositions from LIBS (e.g., Clegg et al. 2009; Tucker et al. 2010; Dyar et al. 2011; Anderson et al. 2011). Eleven rock powder standards (major element compositional statistics are given in Table 8a) were pressed into pellets (Clegg et al. 2009) and ChemCam LIBS observations were made, with fifty laser pulses on each of four different observation points per standard. The first five spectra were ignored and the remaining forty-five spectra from each observation point were averaged. These were corrected for non-laser background and the bremsstrahlung continuum was removed. The PLS analysis used the first five principal components for each element determined by minimizing the global error prediction of the elemental set to be predicted. The 

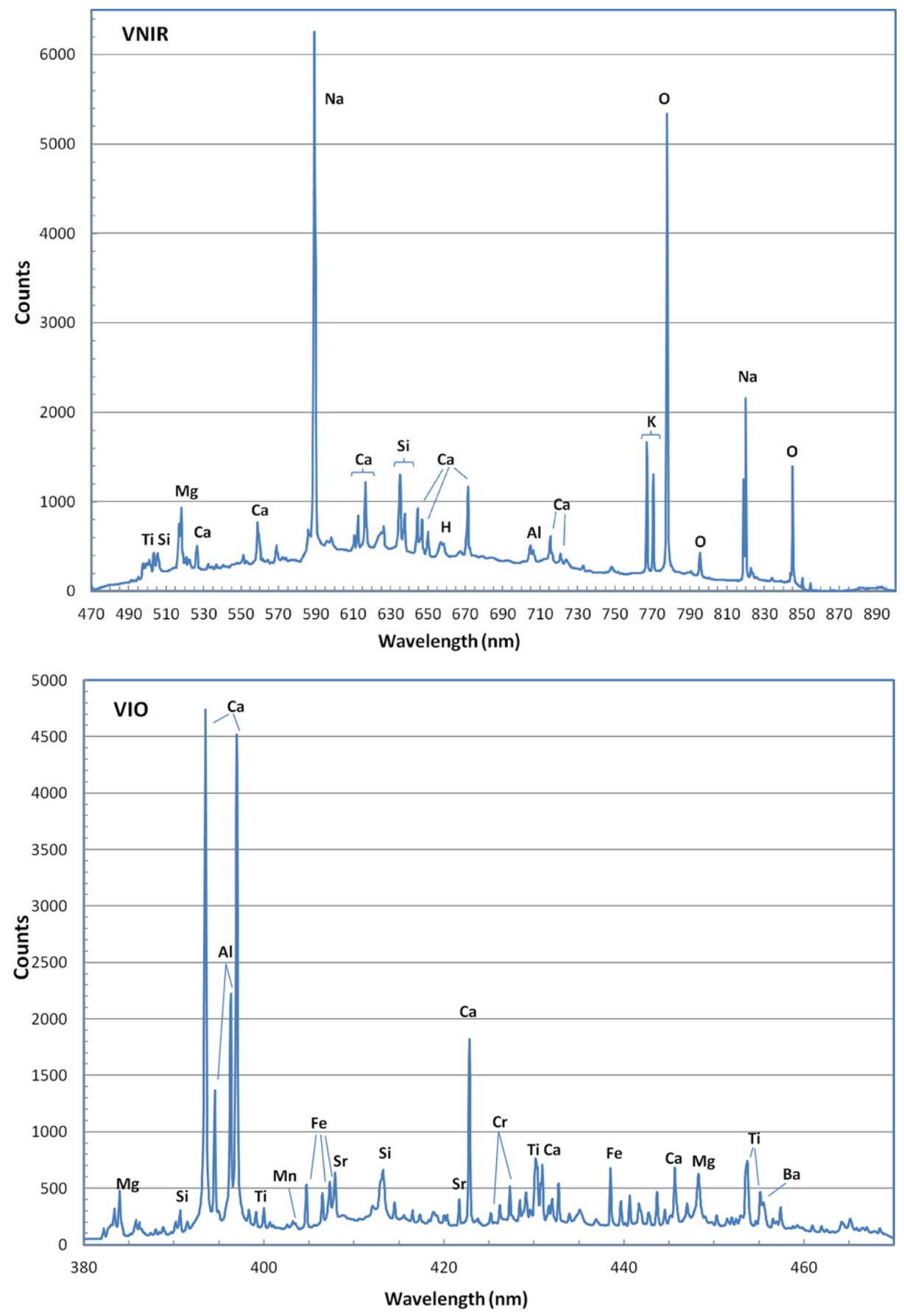

Fig. 25 LIBS spectrum of BT-2 basalt standard at a distance of $3.0 \mathrm{~m}$, showing the VNIR (top), violet (middle), and UV (bottom) spectral ranges. Major peaks are labeled. A non-laser dark spectrum was subtracted, but the continuum was not removed 


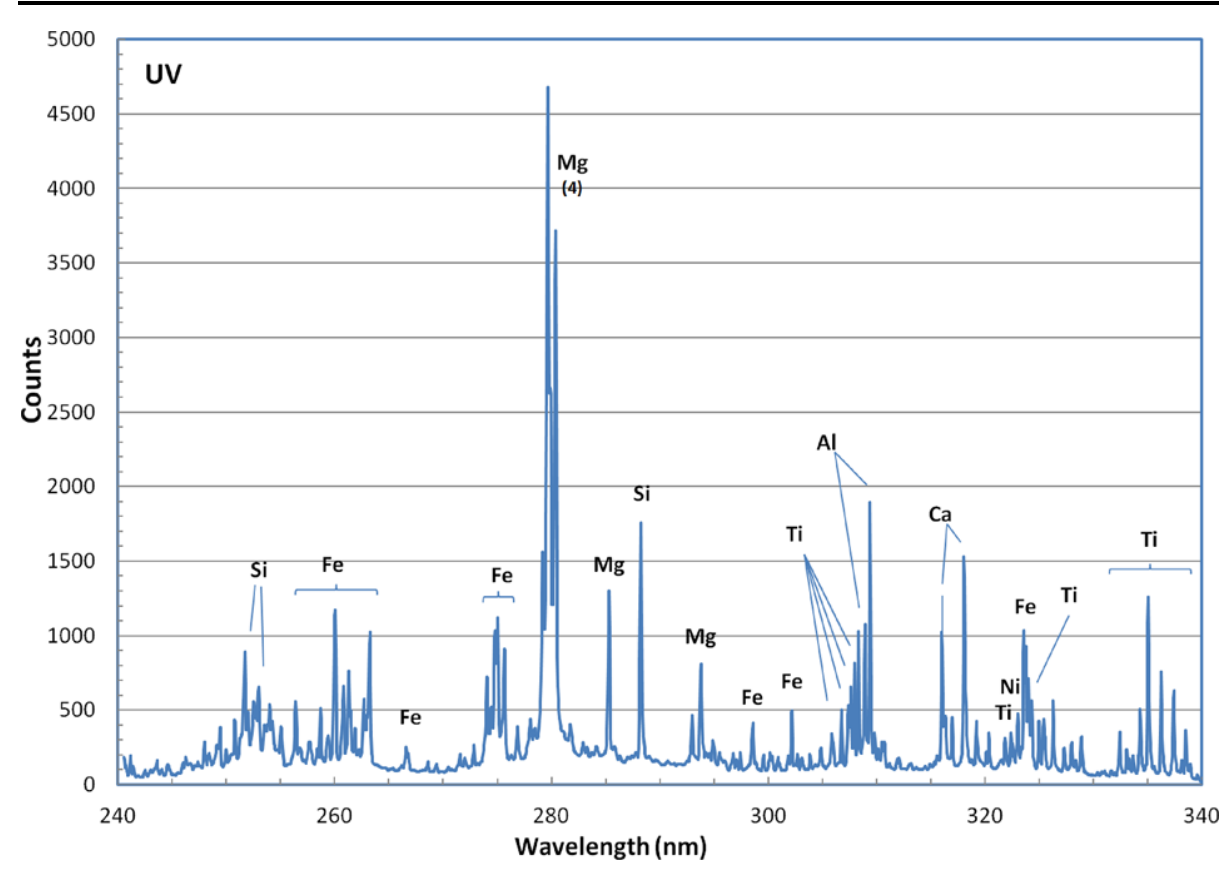

Fig. 25 (Continued)

number of principal components used in the calculation for each element was not optimized, which would have given significantly better results for some of the elements. However, we are looking for a way to predict a priori the optimum number of principal components for a given element, to receive further treatment in a forthcoming calibration paper.

Table 8b-8e presents the results in four different formats: as a median of the relative error prediction of the model (REP; Table $8 b$ ), as a cross validation REP, essentially running PLS forty-four times while one analysis is left out and used as the test case (Table 8c), as a root mean square error prediction (RMSEP; Table 8d) and as a cross-validation RMSEP (Table $8 \mathrm{e}$ ). The accuracy requirement for ChemCam specified less than $\pm 10 \%$ relative error on major elements. For example, a $\pm 10 \%$ relative error on a sample having 50 wt. $\% \mathrm{SiO}_{2}$ means that its composition is predicted with a \pm 5 wt. $\% \mathrm{SiO}_{2}$ accuracy. Tables $8 \mathrm{~d}$ and $8 \mathrm{e}$ show that $\mathrm{SiO}_{2}$ is predicted to much greater accuracy, easily meeting its requirement. ChemCam meets its accuracy requirement for nearly all major elements at all distances based on the RMSEP prediction (Table 8d). However, the cross-validated RMSEP (Table 8e) shows poorer results for the less abundant elements $\mathrm{P}, \mathrm{K}$, Ti, and $\mathrm{Mn}$. A brief comparison between the relative compositional range, defined by the standard deviation divided by the median (Table 8a), and the smallest relative error (RMSEP in Table 8c divided by the median in Table 8 a) for each element shows a strong correlation between relative range and relative error, with an $\mathrm{R}^{2}$ coefficient of 0.89 . This very strongly implies that tighter ranges, especially for $\mathrm{P}$ and $\mathrm{K}$, but also for other elements, would result in significantly reduced errors, implying that the LIBS technique, and the ChemCam instrument in particular, is capable of significantly greater accuracy and precision, given the proper training set. It also suggests that, to achieve the greatest accuracy, the PLS analytical method should ideally be iterative, using a training set with large compositional ranges to first determine the approximate composi- 
Table 8a Compositional range statistics (oxide wt.\%) of the eleven standards used in the multivariate PLS analysis

\begin{tabular}{lcrrrrrrrrr}
\hline & $\mathrm{Na}_{2} \mathrm{O}$ & $\mathrm{MgO}$ & $\mathrm{Al}_{2} \mathrm{O}_{3}$ & $\mathrm{SiO}_{2}$ & $\mathrm{P}_{2} \mathrm{O}_{5}$ & $\mathrm{~K}_{2} \mathrm{O}$ & $\mathrm{CaO}$ & $\mathrm{TiO}_{2}$ & $\mathrm{MnO}$ & $\mathrm{Fe}_{2} \mathrm{O}_{3}$ \\
\hline Minimum & 1.75 & 1.79 & 10.07 & 38.2 & 0.05 & 0.03 & 4.64 & 0.67 & 0.10 & 6.14 \\
Maximum & 4.48 & 13.15 & 17.20 & 62.3 & 1.05 & 3.99 & 13.87 & 2.73 & 0.20 & 14.34 \\
Median & 3.18 & 6.42 & 15.32 & 51.0 & 0.29 & 1.39 & 8.81 & 1.44 & 0.17 & 11.26 \\
Std. Dev. & 0.84 & 3.26 & 1.98 & 7.1 & 0.35 & 1.17 & 3.12 & 0.75 & 0.04 & 2.94 \\
\hline
\end{tabular}

Table 8b Partial least squares (PLS) regression model results for LIBS elemental abundance accuracy for various distances, given as root mean square error prediction in the same units as the oxide abundances (wt.\%)

\begin{tabular}{lllllllllll}
\hline $\begin{array}{l}\text { Distance } \\
(\mathrm{m})\end{array}$ & $\mathrm{Na}_{2} \mathrm{O}$ & $\mathrm{MgO}$ & $\mathrm{Al}_{2} \mathrm{O}_{3}$ & $\mathrm{SiO}_{2}$ & $\mathrm{P}_{2} \mathrm{O}_{5}$ & $\mathrm{~K}_{2} \mathrm{O}$ & $\mathrm{CaO}$ & $\mathrm{TiO}_{2}$ & $\mathrm{MnO}$ & $\mathrm{Fe}_{2} \mathrm{O}_{3}$ \\
\hline 1.6 & 0.10 & 0.40 & 0.38 & 0.43 & 0.03 & 0.13 & 0.17 & 0.10 & 0.00 & 0.39 \\
3.0 & 0.07 & 0.24 & 0.34 & 0.36 & 0.02 & 0.10 & 0.25 & 0.10 & 0.00 & 0.31 \\
5.0 & 0.06 & 0.32 & 0.21 & 0.26 & 0.02 & 0.08 & 0.24 & 0.08 & 0.00 & 0.26 \\
7.0 & 0.05 & 0.41 & 0.23 & 0.30 & 0.02 & 0.06 & 0.20 & 0.08 & 0.00 & 0.22 \\
\hline
\end{tabular}

Table 8c PLS regression model results for LIBS elemental abundance accuracy for various distances, leaving one analysis out and constructing a model with the remaining measurements to predict the one left out. Results are given as root mean square error in the same units as the oxide abundances (wt.\%)

\begin{tabular}{lcccccccccc}
\hline $\begin{array}{l}\text { Distance } \\
(\mathrm{m})\end{array}$ & $\mathrm{Na}_{2} \mathrm{O}$ & $\mathrm{MgO}$ & $\mathrm{Al}_{2} \mathrm{O}_{3}$ & $\mathrm{SiO}_{2}$ & $\mathrm{P}_{2} \mathrm{O}_{5}$ & $\mathrm{~K}_{2} \mathrm{O}$ & $\mathrm{CaO}$ & $\mathrm{TiO}_{2}$ & $\mathrm{MnO}$ & $\mathrm{Fe}_{2} \mathrm{O}_{3}$ \\
\hline 1.6 & 0.50 & 0.98 & 1.43 & 1.75 & 0.15 & 0.50 & 0.82 & 0.45 & 0.03 & 1.37 \\
3.0 & 0.54 & 1.06 & 1.47 & 1.64 & 0.13 & 0.41 & 0.90 & 0.37 & 0.03 & 1.55 \\
5.0 & 0.40 & 1.18 & 1.55 & 1.54 & 0.14 & 0.39 & 0.89 & 0.32 & 0.03 & 1.61 \\
7.0 & 0.51 & 1.13 & 1.55 & 1.80 & 0.14 & 0.56 & 0.82 & 0.46 & 0.03 & 1.60 \\
\hline
\end{tabular}

Table 8d PLS regression model results for LIBS relative elemental abundance accuracy (in \%) for various distances, given as medians of relative error predictions

\begin{tabular}{lllllrrrrrr}
\hline $\begin{array}{l}\text { Distance } \\
(\mathrm{m})\end{array}$ & $\mathrm{Na}_{2} \mathrm{O}$ & $\mathrm{MgO}$ & $\mathrm{Al}_{2} \mathrm{O}_{3}$ & $\mathrm{SiO}_{2}$ & $\mathrm{P}_{2} \mathrm{O}_{5}$ & $\mathrm{~K}_{2} \mathrm{O}$ & $\mathrm{CaO}$ & $\mathrm{TiO}_{2}$ & $\mathrm{MnO}$ & $\mathrm{Fe}_{2} \mathrm{O}_{3}$ \\
\hline 1.6 & 3.2 & 7.4 & 2.7 & 0.78 & 13.2 & 12.0 & 2.1 & 6.1 & 2.5 & 3.9 \\
3.0 & 2.5 & 4.2 & 2.4 & 0.69 & 9.3 & 8.8 & 2.5 & 7.0 & 2.1 & 3.7 \\
5.0 & 1.7 & 4.8 & 1.5 & 0.49 & 6.5 & 7.3 & 2.8 & 5.7 & 2.6 & 2.6 \\
7.0 & 1.8 & 5.6 & 1.5 & 0.60 & 8.3 & 6.0 & 2.6 & 6.5 & 2.4 & 2.7 \\
\hline
\end{tabular}

tion of the unknown and then potentially using a training set with only small compositional ranges matching the expected composition of the unknown.

An interesting detail of Tables $8 \mathrm{a}-8 \mathrm{e}$ is that there is no strong trend as a function of sample distance even though the signals at $7 \mathrm{~m}$ are more than an order of magnitude below those obtained at the closest distances. This suggests that the uncertainties are dominated by 
Table 8e PLS regression model results for LIBS relative elemental abundance accuracy (in \%) for various distances, leaving one analysis out and constructing a model with the remaining measurements to predict the one left out. Results are given as medians of relative error predictions

\begin{tabular}{llrllllllll}
\hline $\begin{array}{l}\text { Distance } \\
(\mathrm{m})\end{array}$ & $\mathrm{Na}_{2} \mathrm{O}$ & $\mathrm{MgO}$ & $\mathrm{Al}_{2} \mathrm{O}_{3}$ & $\mathrm{SiO}_{2}$ & $\mathrm{P}_{2} \mathrm{O}_{5}$ & $\mathrm{~K}_{2} \mathrm{O}$ & $\mathrm{CaO}$ & $\mathrm{TiO}_{2}$ & $\mathrm{MnO}^{\mathrm{Fe}_{2} \mathrm{O}_{3}}$ \\
\hline 1.6 & 7.7 & 11.6 & 6.6 & 2.0 & 29.8 & 18.7 & 6.8 & 15.0 & 11.8 & 8.9 \\
3.0 & 8.9 & 8.9 & 3.0 & 1.8 & 26.8 & 21.4 & 4.8 & 21.3 & 14.1 & 10.1 \\
5.0 & 7.9 & 9.2 & 4.9 & 1.6 & 28.4 & 21.9 & 4.0 & 18.4 & 14.0 & 13.9 \\
7.0 & 7.0 & 10.5 & 2.9 & 2.4 & 28.3 & 26.1 & 4.5 & 17.0 & 11.6 & 12.1 \\
\hline
\end{tabular}

shot-to-shot variations and by details related to the training set rather than by low signal-tonoise ratios. However, at distances greater than $7 \mathrm{~m}$ observable emission peaks are rapidly lost.

Other PLS studies of LIBS data have used much larger numbers of standards than the eleven used here, leading to lower overall errors (e.g., Clegg et al. 2009; Tucker et al. 2010; Dyar et al. 2011; Anderson et al. 2011). A large training set is planned to be used during operations on Mars, to be analyzed with laboratory models similar in design to ChemCam. More complete details of the standards used here, the data treatment, PLS and classification results, and detection limits for various elements will be given in a forthcoming paper on ChemCam LIBS calibration.

Sample classification is expected to be an important task for ChemCam. From a scientific standpoint, elemental compositions are important, but from a tactical, day-to-day standpoint, determining whether a sample is different than previously encountered will be the first priority. The data sets generated from the experiments listed in Table 7 have demonstrated the ability to classify samples using independent components analysis. Cousin et al. (2011a) tested a classification scheme using ICA in which they re-divided the four observation points each having fifty laser pulses (Table 7, \#1) and averaged groups of ten individual spectra so there were twenty averaged spectra to classify for each standard. Using the Jackknife method they were able to classify $100 \%$ of the spectra as belonging to the correct sample with using relatively few components when the model and unknowns were all measured at the same distance. When classifying samples analyzed at a different distance than the samples used for the model, classification accuracy dropped to $87.5 \%$. Correction of spectra for different distances is currently a work in progress and requires accurate removal of the electron continuum.

Ollila et al. (2012) tested two different classification schemes using fifty-six of the standards analyzed by ChemCam at $3.0 \mathrm{~m}$ in the large group of standards (row 6 in Table 7). Her study compared traditional partial least squares discriminant analysis (PLS-DA) to a classification and removal-based predictive flow developed by Multari et al. (2010; "modified PLS-DA"). This work showed that the modified algorithm performed better than the traditional technique, achieving $100 \%$ correct classification using a training data set and $95 \%$ correct on an unknown data set, compared to $63 \%$ for the traditional PLS-DA. This is exceptionally good considering the large number of classes and the geological similarities of many of the samples.

The above classification schemes typically use multiple dimensions (components), making visualization of the differences between classes difficult. For two-dimensional visualization, Lasue et al. (2011) tested a Sammon's map algorithm, which is a non-linear technique based on an iterative approach to find the global minimum of the stress function by following a steepest descent procedure. This study used the twenty-six standards analyzed by 
Fig. 26 Test of LIBS profile through thick dust. Loose dolomite dust was placed on a pressed basalt target, lying at an $\sim 45^{\circ}$ angle. Circle indicates the pit created by the laser pulses

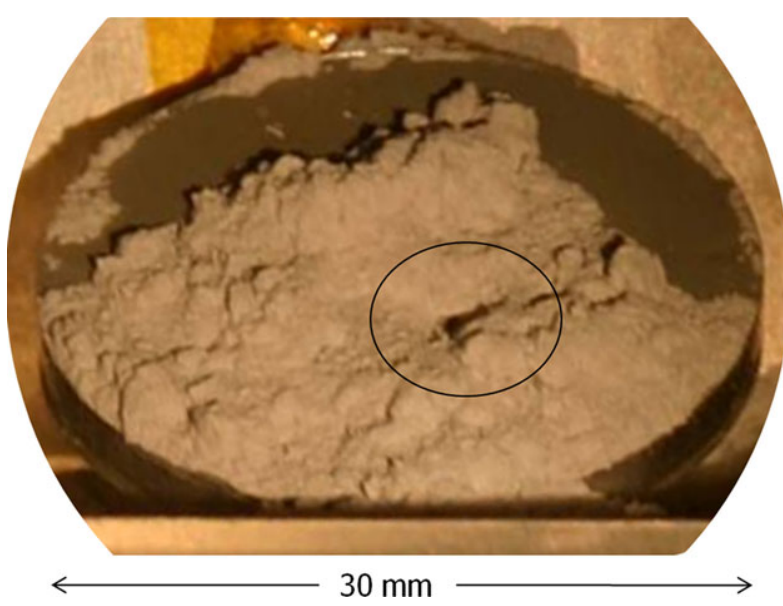

ChemCam at $3.0 \mathrm{~m}$ in the thermal vacuum test (Table 7, \#1) to demonstrate that for simple visualization, i.e., two-dimensional representation, a Sammons map algorithm is the best tool, resulting in entropy values (a measure of clustering quality) less than half those of maps produced by ICA and principal component analysis (PCA).

A difference from real-world samples exists in that all of the targets used in ChemCam pre-delivery analyses were powders pressed together using 35 tons of force. These were used in order to avoid heterogeneity, so that the actual uncertainties inherent in the technique could be determined. On Mars and for other geological applications the heterogeneity of rock samples must be taken into account.

\subsection{Dust Removal}

An important feature of LIBS is the ability to remove dust coatings from rocks so the measured composition is not obscured or contaminated. This capability is a significant advantage over passive remote observations, where a layer that is a few tens of $\mu \mathrm{m}$ thick can obscure visible spectra and one to two hundred microns can obscure a thermal IR spectrum (Morris et al. 2001; Graff et al. 2001). Experiments were conducted to confirm and characterize this capability using multiple laser pulses: One experiment each addressing thick and thin dust coatings (\#9 and 10 in Table 7). To address the case of thick dust a pressed powder disk of basalt standard GBW07105 was used as the "rock". It was positioned at $\sim 45^{\circ}$ from the horizontally-directed beam. Dolomite $\left(\mathrm{CaMg}\left(\mathrm{CO}_{3}\right)_{2}\right)$ powder standard JDo-1 was sprinkled on the surface to $>1 \mathrm{~mm}$ thickness, as shown in Fig. 26. The arrangement was positioned $3.5 \mathrm{~m}$ from the instrument and in 7 Torr of $\mathrm{CO}_{2}$ to simulate the Mars atmosphere. The laser was pulsed fifty times at $3 \mathrm{~Hz}$ and the individual spectra were recorded.

The data were processed by averaging successive groups of five spectra. The results are shown in Fig. 27 for the $288 \mathrm{~nm} \mathrm{Si} \mathrm{emission} \mathrm{line.} \mathrm{The} \mathrm{initial} \mathrm{five} \mathrm{spectra} \mathrm{show} \mathrm{no}$ contribution from the underlying basalt, as indicated by the absence of the $\mathrm{Si}$ emission line. The next two averages of five show that the basalt has been sampled at least by pulse number ten, with increasing signal from pulses eleven to fifteen. Interestingly, pulses sixteen through twenty show decreased Si emission due most likely to dolomite dust falling into the hole created by the plasma, given the angle of repose and the size of the hole shown in Fig. 26. Finally, pulses 21-25 show the highest signal, which stayed constant in all successive pulses (not shown), indicating that infall of dust no longer occurred. 
Fig. 27 Successive

five-spectrum averages showing the $288 \mathrm{~nm}$ Si emission line strength as the laser profiles through the $\mathrm{Si}$-free dust to the Si-containing basalt at the analysis location shown in the previous figure. The low peak for laser shots 16-20 likely results from powder falling back into the hole

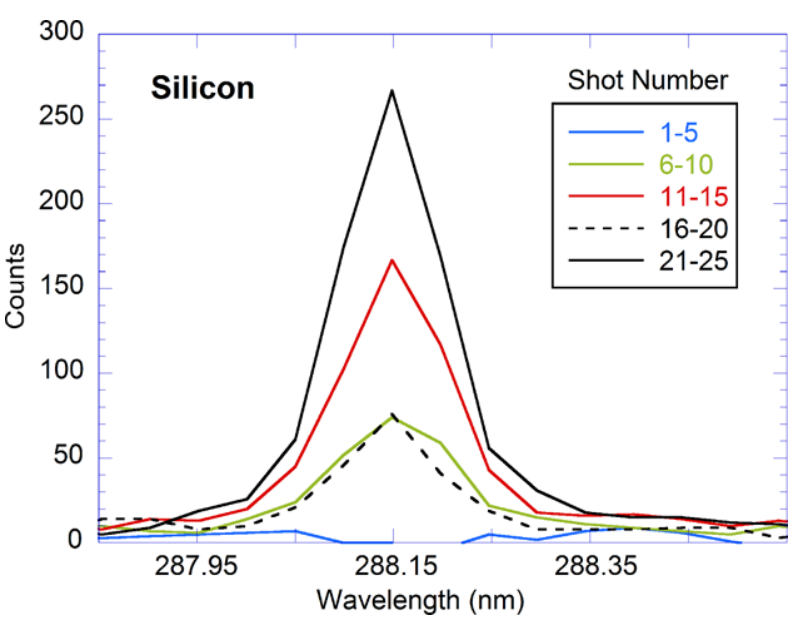

The experiment was repeated with a thin dust layer more likely representative of the typical dust layer on Mars rocks. For this aspect the beam was directed horizontally downward with a turning mirror and the chamber which was positioned on the floor was de-pressurized to 7 Torr. The sample distance was $2.9 \mathrm{~m}$ and the beam was normal to the surface. JDo- 1 powder was again used for the dust, and this time the underlying basalt was a sawed slab from Broken Tank, NM (BT-2). The dust was leveled at a thickness of $25(+20,-10) \mu \mathrm{m}$. First, five pulses at a laser energy of $7.5 \mathrm{~mJ}$ were applied. The cleared portion of the sample was examined. The sample was then moved to create a new observation point with 10 pulses of $10 \mathrm{~mJ}$. The sample was again moved and a third observation point was created with 50 pulses of $10 \mathrm{~mJ}$. (Recorded laser energies do not take into account attenuation by the mirror and sample chamber window.)

Figure 28 is an RMI image of the sample after all three observations. The diameters of the exposed areas on the basalt range from 1.5 to $2.4 \mathrm{~mm}$. Figure 29 shows a portion of the UV spectrum from each of the first five laser pulses at $7.5 \mathrm{~mJ}$. Spectra one and two, at the bottom of the figure, consist almost entirely of $\mathrm{Mg}$ and $\mathrm{Ca}$ emission lines. The strongest emission lines of carbon are not in this portion of the spectrum. The dolomite has a clean baseline, indicating the relative absence of transition elements which have a great number of emission lines. Spectra four and five, near the top of the figure, show emission lines typical of basaltic compositions. The spectra are characterized by multiple iron emission lines near $265 \mathrm{~nm}, \mathrm{Mg}$ peaks near 280 and $285 \mathrm{~nm}$, a strong Si peak at $288 \mathrm{~nm}$, Ti peaks near 305$308 \mathrm{~nm}$ (cf. Fig. 25), Al peaks at 308 and $309 \mathrm{~nm}$, and Ca peaks near 316 and $318 \mathrm{~nm}$. Spectrum three (Fig. 29) is a transition between the dolomite and the basalt compositions. Interestingly, this transition spectrum is a factor of three to eight times weaker in intensity in terms of the highest peak $(\mathrm{Mg}$ at $280 \mathrm{~nm})$ than the other spectra.

\subsection{Soil Analysis Simulation}

A test was made to simulate taking LIBS spectra of soil (\#11 in Table 7). For this test the same vertical beam set-up and instrument-to-sample distance was used as in Figs. 28 and 29. A jar filled more than $5 \mathrm{~cm}$ deep with dolomite standard JDo-1 was placed in the chamber. The grain size of JDo-1 is relatively fine, with less than $2 \%>90 \mu \mathrm{m}$ and more than $73 \%<20 \mu \mathrm{m}$ (R.V. Morris, personal communication). The chamber was pumped to achieve a comparable ambient pressure to that on Mars. Fifty laser pulses of $\sim 10 \mathrm{~mJ}$ each 
Fig. 28 RMI image of white dust-coated basalt at $2.9 \mathrm{~m}$ under Mars atmospheric pressure with pulses of $7.5 \mathrm{~mJ}$ (right), 10 pulses of $10 \mathrm{~mJ}$ (below), and 50 pulses of $10 \mathrm{~mJ}$ (above). The slab is $42 \mathrm{~mm}$ across LIBS observations using 10 laser

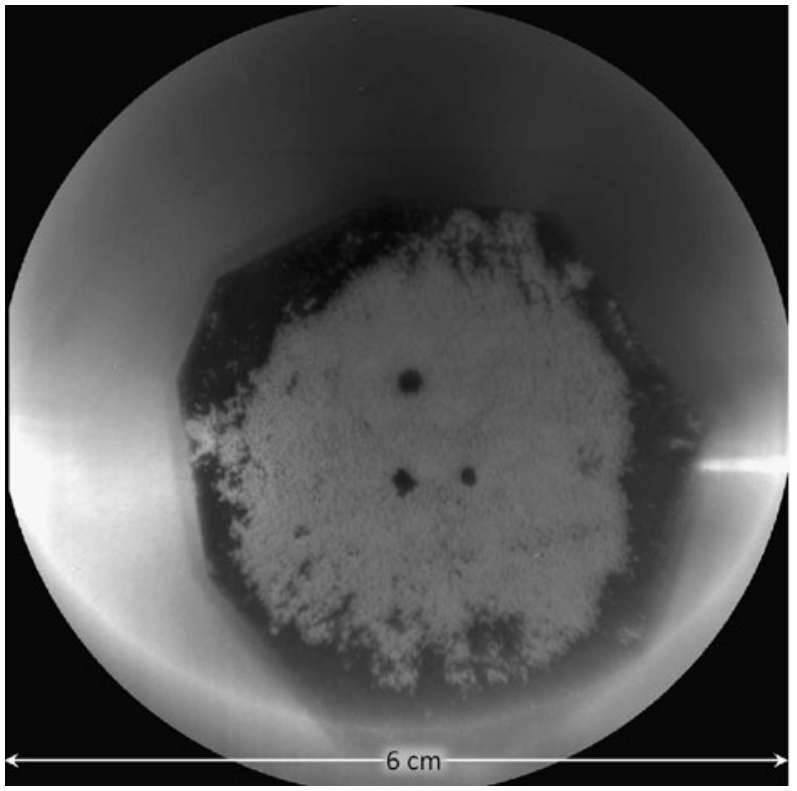

Fig. 29 Evolution of a portion of the UV spectrum over laser pulses one to five of a thin layer of dolomite powder resting on a basalt slab, shown as the observation point on the right in the previous figure. Laser pulse number is given on left. The spectra corresponding to laser pulses one and two show essentially only dolomite, the spectrum from pulse three indicates a transition, with the spectra from pulses four and five appearing typical of basalt

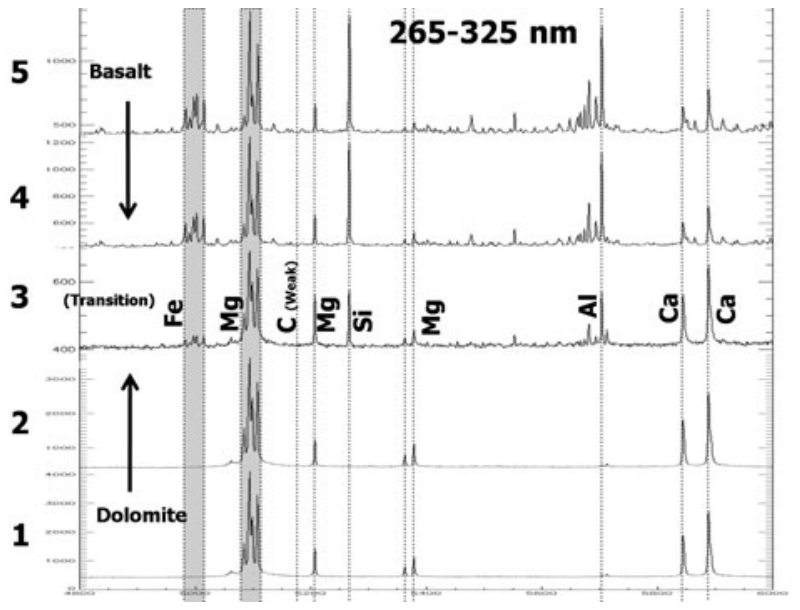

were directed at the powder and each spectrum was collected individually. After photo documentation an additional one hundred pulses were applied. A hole of $1.4 \mathrm{~mm}$ diameter and unknown depth was produced by the first set of fifty pulses and was widened to $1.8 \mathrm{~mm}$ after one hundred fifty pulses. A rim of slightly lighter appearing material was formed around the hole by the first burst of pulses and its size and color contrast were increased by the second burst. The rim was most likely formed from finer-grained material removed from the hole.

Examination of the spectra showed that the amplitude of the emission lines decreased by more than a factor of four between the first two pulses, averaged, and the $49^{\text {th }}-50^{\text {th }}$ pulses, as shown in Fig. 30. However, the widths of the peaks did not decrease as might be expected. The resulting increases in FWHM of the peaks are labeled in Fig. 30, and vary between relatively small increases and changes of $>100 \%$. The variable reduction in peak height 


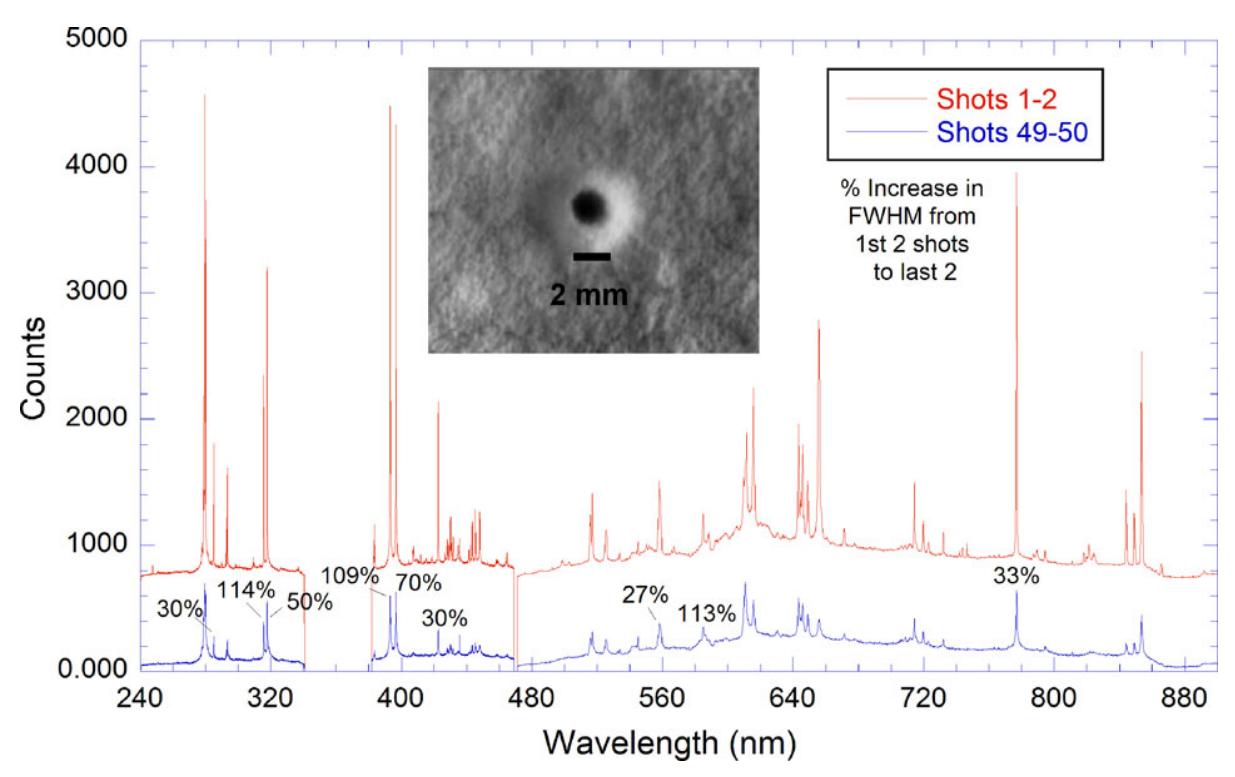

Fig. 30 Spectra of first two and 49th and 50th laser pulses (averaged respectively) in JDo-1 powder. Numbers indicate the percent increase in FWHM of various peaks due to self absorption. Inset shows an RMI image subframe of the hole produced by one hundred fifty pulses

without corresponding width reduction is indicative of self absorption within the plasma. This occurs when emission-line photons within by non-excited atoms within an optically thick plasma (Lazic et al. 2001). It may have occurred in this case due to the depth and narrow dimensions of the hole causing a thicker plasma, though none of the peaks display a central depression which is a hallmark of strong re-absorption. Because a portion of the signal is attenuated, and because PLS uses spectrally narrow detector channels to correlate with elemental compositions, the relatively flat, broad peaks of self-adsorbed material result in inaccurate compositional results. To obtain quantitative compositional results the FWHM should be checked to indicate self absorption, which should be avoided.

Soils on Mars are likely to vary in morphology and have a range of particle sizes; duricrusts are also present (e.g., Sullivan et al. 2008). The lower gravitational potential on Mars may lead to more rapid excavation of holes in soil material similar to that tested here. It will therefore be important to use smaller numbers of pulses to avoid self absorption.

\subsection{Depth Profiles in Rock}

ChemCam is capable of observing weathering layers on rock targets, and, by removing this layer, determine the chemical composition of the underlying rock (e.g. Wiens et al. 2004; Sharma et al. 2007; Lanza et al. 2012). To test ChemCam's depth profiling capability (\#3 in Table 7), a sawed slab of Grapevine Spring black calcite vein (Vaniman et al. 1995) and of basalt were each subjected to a series of 500 and 1000 laser pulses in separate locations. The calcite was interrogated at $3 \mathrm{~m}$, while the basalt was targeted at both 3 and $7 \mathrm{~m}$. The samples were surrounded by 7 Torr of air to simulate the Mars atmospheric pressure.

The results are shown in Table 9. The depths of the pits were measured with a calibrated Nikon MM-60 microscope. The 500-pulse pit in the basalt at $3 \mathrm{~m}$ was measured to be $600 \mu \mathrm{m}$ in diameter. The deepest pit was observed to be $>560 \mu \mathrm{m}$ made by 1000 pulses 
Table 9 LIBS depth profile results

\begin{tabular}{llrl}
\hline Rock & Distance & \# Pulses & Maximum depth \\
\hline Basalt & $3 \mathrm{~m}$ & 500 & $410 \mu \mathrm{m}$ \\
Basalt & $3 \mathrm{~m}$ & 1000 & $300 \mu \mathrm{m}$ \\
Basalt & $7 \mathrm{~m}$ & 500 & $240 \mu \mathrm{m}$ \\
Basalt & $7 \mathrm{~m}$ & 1000 & $330 \mu \mathrm{m}$ \\
Calcite & $3 \mathrm{~m}$ & 500 & $400 \mu \mathrm{m}$ \\
Calcite & $3 \mathrm{~m}$ & 1000 & $>560 \mu \mathrm{m}$ \\
\hline
\end{tabular}
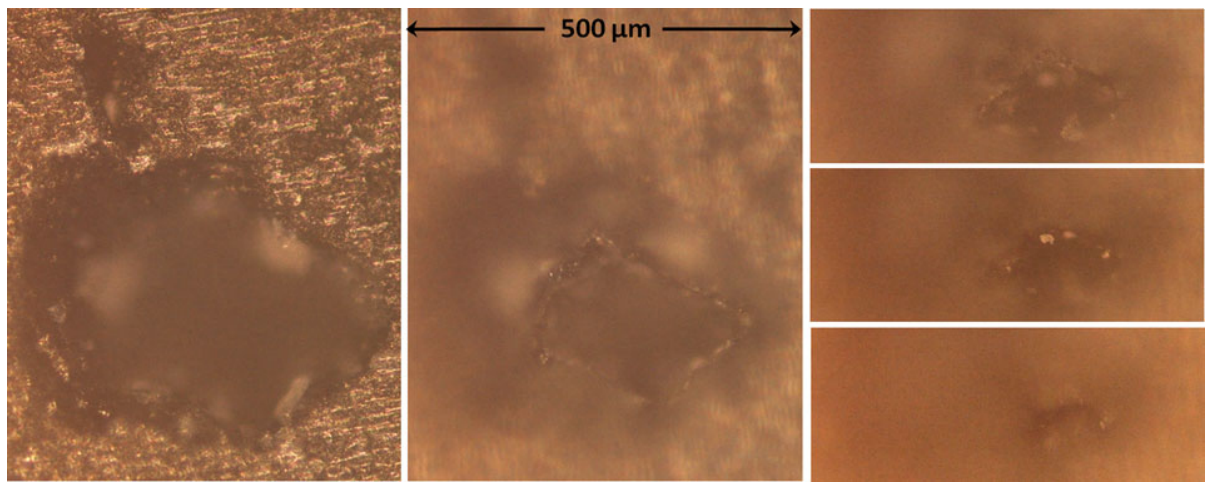

Fig. 31 Microscope images of a 500-pulse depth profile into calcite at $3 \mathrm{~m}$ distance, taken with successively deeper focus offsets. The image on the left shows the saw-cut surface of the sample in focus; the images at the lower right was taken with an offset of $560 \mu \mathrm{m}$, showing the deepest portion of the pit. All images are at the same scale

in the calcite at $3 \mathrm{~m}$. The slab was mounted a few degrees from normal for the laser pulses, which made it difficult to see all the way to the bottom of the pit with the microscope. Successive microscope images of the 500 pulse pit in calcite are shown in Fig. 31. The pit diameter clearly decreases with depth to a small cross section near the deepest portion of the pit.

The experiment should have used more laser pulses. However, the project was near its limit of pre-launch pulses permitted for ChemCam, so only 4500 pulses were expended on this test. Depth profiles can normally use several thousand pulses each, but for this experiment several pits of different numbers of laser pulses were desired. So the depths of these laser pits were each less than should be feasible with ChemCam. Additional experiments are being conducted (e.g., Cousin et al. 2011b) or planned with different types of rock targets and with terrestrial weathering layers from different environments, using laboratory set-ups similar to ChemCam.

\section{ATLO Testing}

After delivery of the completed instrument to JPL, testing of ChemCam during the rover assembly, test, and launch operations (ATLO) phase focused mostly on (a) determining optical transmission with the flight fiber optic cable, as this was the first time the instrument was mated to it, (b) ensuring the proper operation of flight software in controlling ChemCam, 
(c) characterizing ChemCam in the environment of the rover, i.e., with the flight RWEB window, with the calibration targets as mounted on the rover, and using the articulation of the RSM, and (d) post-delivery and pre-close-out final tests to make sure that the instrument was working properly. In addition, ATLO testing provided one final opportunity to observe ChemCam operation under more Mars-like environments.

Transmission tests with the flight optical fiber and with the RWEB window indicated that these components were well characterized, with results as expected.

ChemCam operations were conducted during the rover system thermal test (STT), which followed the rover vibration test and so served as verification for that test as well. In addition to using the on-board calibration target assembly, two panels populated with rock slabs and a titanium plate were mounted at distances of $\sim 2.7$ and $\sim 4.7 \mathrm{~m}$ from the MU aperture. Samples were from R.V. Morris' collection and some had been used for calibration of optical instruments for previous Mars missions (e.g., Squyres et al. 2003). Most of the sample analyses during STT exercised a standard line scan with five LIBS analyses of thirty laser pulses each, placed within one milliradian of each other, accompanied by a non-laser dark measurement of fifty spectra. All of the data were downlinked as individual spectra. Samples analyzed using five-point line scans at the $\sim 4.7 \mathrm{~m}$ distance included three different basalts and a trachyte. Samples analyzed at $\sim 2.7 \mathrm{~m}$ included four different basalts, a trachyte, a banded iron formation sample, and a titanium plate, which was targeted at normal laser energy (95 A) and at lower energy (60 A). Images and post-test inspection of the analysis points did not show any difficulty with maintaining the pointing during the LIBS analyses. Based on ATLO testing the pointing accuracy is expected to be within $\pm 5 \mathrm{mrad}$. Accuracy from a given point to the next point is much better, allowing inter-point spacing to be as small as $0.2 \mathrm{mrad}$. Pointing stability under actual Mars conditions, for example under various wind speeds, will need to be checked after landing.

The banded iron sample (BIFAUS2) observed at $\sim 2.7 \mathrm{~m}$ demonstrates the utility of LIBS in observing rock strata and laminae, as expected in Gale Crater's layered mound. Banded iron formations are Precambrian sedimentary rocks in which oxidized iron alternates with silica-rich material in layers $\mathrm{mm}$ to a few $\mathrm{cm}$ thick (e.g., Klein 2005). Figure 32 shows the RMI image of the BIF just prior to and after a line scan of five points, and also shows the huge variation in spectra between iron-rich and silica-rich layers. The silica-rich point came from the bright region of the image and inset. Martian strata will likely have more subtle spectral variations. Figure 32 also shows the relative spacing capability of the points.

Work is underway to produce elemental composition predictions from the STT LIBS data using the training set of standards analyzed during LANL calibrations, and to compare these to the actual compositions. This may require masking some spectral regions, e.g., those containing nitrogen, oxygen, and carbon emission lines, due to the nitrogen atmosphere during STT.

Passive spectra were taken of the Ti plate and ceramic standard NAu-2-Hi-S (\#10 and $\#$, respectively in Fig. 3) during STT using the LIBS spectrometers without the laser and with exposures on the order of $1 \mathrm{~s}$. However, the incident illumination was uncalibrated and did not closely resemble solar illumination, particularly in the UV.

As a final note on LIBS, and to highlight a capability that will likely be important for understanding the role of water on Mars, it was noticed (A. Ollila, personal communication) that for all of the standards analyzed prior to instrument delivery, the first one or several spectra showed enhanced hydrogen peaks at $656 \mathrm{~nm}$. This is presumably from water vapor adsorbed on the surface of the sample even though the samples were contained in a chamber with 7 Torr of $\mathrm{CO}_{2}$ to simulate the Mars atmosphere. While the pre-delivery standards consisted of pressed powders, which should have a high surface area to facilitate condensation 

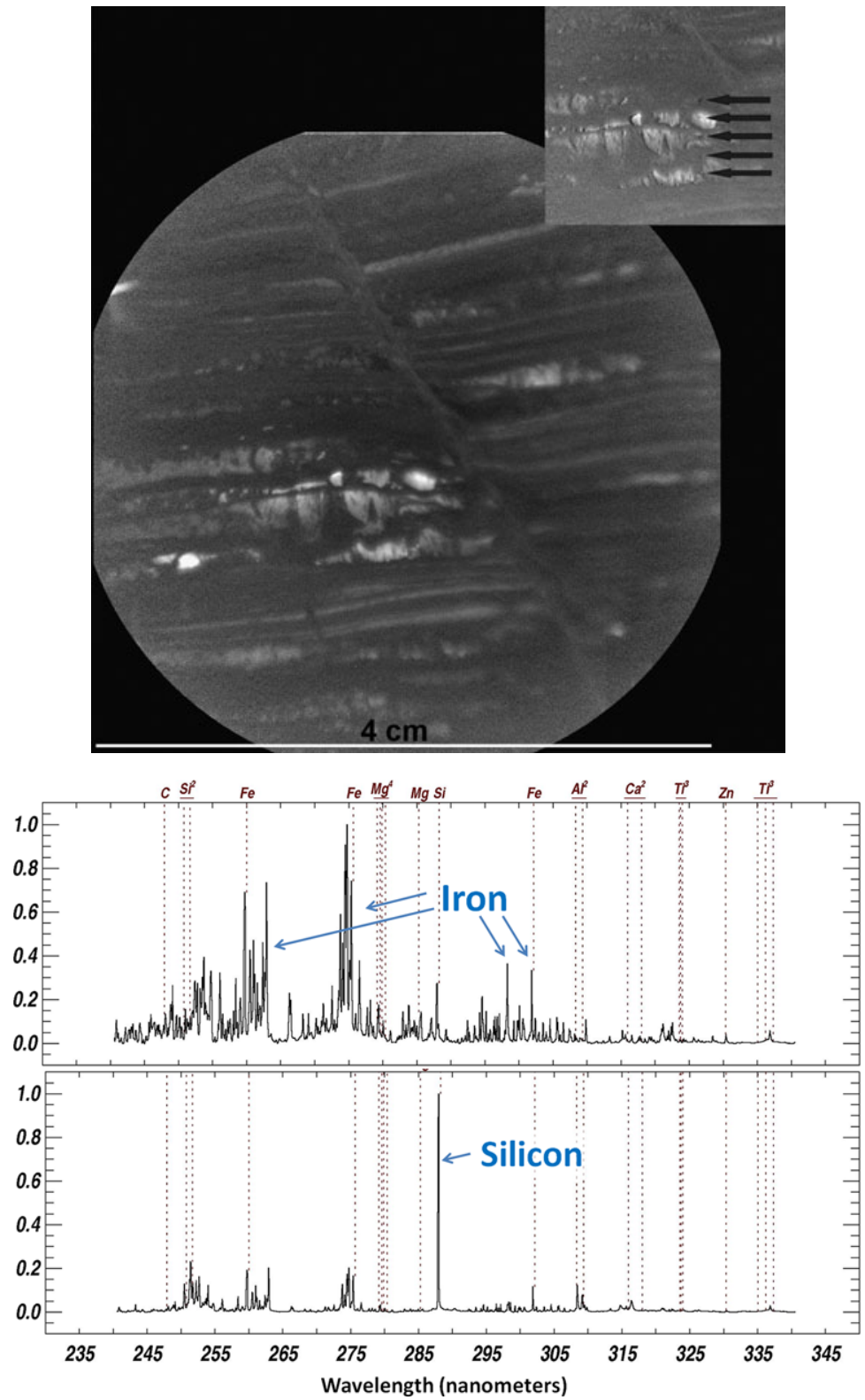

Fig. 32 RMI image, taken under relatively low ambient light conditions, of banded iron formation at a distance of $2.7 \mathrm{~m}$ before acquiring LIBS spectra (top) and after LIBS (inset). Arrows point to the five observation points, each separated by $\sim 2 \mathrm{~mm}$. The UV portion of the spectrum is shown for two different observation points (bottom). A LIBS quick-look data product image is used, showing normal positions of major peaks, not all seen in these panels

of water, the samples used in STT all consisted of rock slabs. The STT spectra, where the large chamber containing the rover and the targets was filled with $\sim 10$ Torr of nitrogen, showed that the phenomenon persisted there as well. Figure 33 shows the VNIR spectral region containing the $656 \mathrm{~nm} \mathrm{H}$ peak, and shows the augmented $\mathrm{H}$ signal on the first two 
Fig. 33 The $656 \mathrm{~nm} \mathrm{H}$ peak region for the first five spectra of a basaltic andesite rock slab observed at $4.7 \mathrm{~m}$ distance during rover system thermal testing in JPL's large thermal chamber in $\sim 10$ Torr of nitrogen, showing the enrichment of $\mathrm{H}$, presumably from water molecules adsorbed on the surface, typically seen on all samples during the initial laser pulses even after one week at low pressure

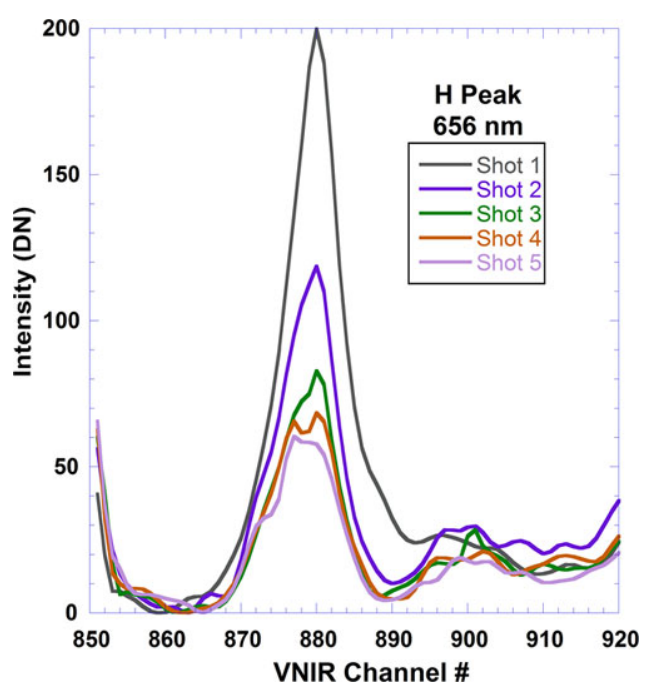

laser pulses for a random STT sample, a basaltic andesite from Sisters, OR, provided by R.V. Morris. The observation was made on 19-March, 2011, at least a week after the beginning of the test, when the chamber temperature was above $0{ }^{\circ} \mathrm{C}$. A concerted effort will need to be made to understand the amount of water represented by these signals. Frost was observed (Landis 2007) by the MER-A rover at Gusev Crater, which is in a similar equatorial latitude to Gale Crater. However, it is difficult to quantify the amount of water deposited based only on PanCam imaging. ChemCam observations, made early in the morning, will indicate the amount of water condensed overnight at various times of the year in Gale crater.

\section{Summary and Conclusions}

The ChemCam instrument package, including the first LIBS spectrometer for planetary exploration as well as a high resolution context imager, was launched 26-November, 2011 and is due to land on 5-August, 2012. ChemCam represents a new type of remote sensing which can actively interrogate samples at a distance with the use of repeated laser pulses. Here we revisit the five planned capabilities of the instrument suite to provide a final assessment of expected performances:

Rapid Rock Identification Classification tests mentioned above yielded excellent results for ChemCam, however, these tests used pressed rock powders, so they were not an ideal test for real conditions. The results on Mars will likely depend on the homogeneity of the materials being analyzed. Variations in classification results on more heterogeneous samples may actually indicate spatial trends in mineral assemblages. Accurate results also depend on the library of reference spectra that serve as a model. There is ongoing work at LANL and IRAP as well as elsewhere to expand the variety of ChemCam reference spectra, to correct them for instrument response and laser and environmental conditions, and to use them with the optimum classification tools based on various clustering methods. 
Quantitative Analyses The goal for quantitative elemental compositions was a relative error of $\leq 10 \%$. Table $8 \mathrm{~d}$ shows that the model results achieved the desired goal, however, when tested in a leave-one-out routine (Table 8e), errors on $\mathrm{P}, \mathrm{K}, \mathrm{Ti}, \mathrm{Mn}$, and Fe exceeded $10 \%$ relative uncertainty. Overall, results tended to trend from accurate results for the most abundant element, $\mathrm{Si}$, which showed leave-one-out errors $\sim 2 \%$, to higher errors for the less abundant elements. Multivariate analyses rely on an ensemble of standards, called a training set, that is similar in composition to the unknowns. A strong correlation between relative range of a given element in the training set and its relative error suggests that iterative application of MVA to use standards that more closely match the unknown will significantly increase the accuracy. Alternately, other studies strongly suggest that increasing the number of standards used in the training set will significantly increase the accuracy as well. The required training set is much larger than the set of onboard standards. On Mars ChemCam has two options for training sets of similar standards: One is to cross calibrate with compositions analyzed by APXS and CheMin and to use these samples as the training set. However, this will not work for elements such as Li which are detected exclusively by LIBS. For these, comparison with a ground-based training set is necessary. The pre-delivery calibrations comprise one set of standards that can be used for this training set. To ensure that these can be directly compared, efforts must be made to understand any changes in the instrument response over time. It will also be important to correct for the effects of analyzing samples at different distances from the instrument.

Depth Profiles Pre-delivery characterization demonstrated that ChemCam LIBS can remove a typical dust layer in as few as three laser pulses, and it can remove a thick $(>1 \mathrm{~mm})$ dust layer in fifteen pulses. The requirement to profile $>1 \mathrm{~mm}$ in rock samples was not conclusively met, as analyses with 1000 pulses stopped at $<600 \mu \mathrm{m}$, though it is likely that 2000 pulses would have achieved the requirement. More studies are needed, perhaps with synthetic layered materials of hardnesses similar to that encountered in nature, to understand the amount of mixing between different layers apparent in the spectra as the center of the beam profiles deeper while the periphery of the pit is less deep.

Context Imaging ChemCam RMI meets its technical requirements for providing context imaging. Verification is provided in the companion paper (Maurice et al. 2012). The RMI resolution matches that of the highest resolution imager to date on the surface of Mars. In this context the focus quality is a critical aspect of the imaging capability. The RMI depth of focus is narrow, consequently fields which exceed this depth will need to be imaged using Z-stacks.

Passive Spectroscopy While not calibrated specifically as a passive system, given that the instrument response is well characterized, passive spectroscopy over the spectral range of 240-905 nm should be useful in at least two ways: First, the UV region of the spectrum is unique to ChemCam among Mars instruments. Cloutis et al. (2008) have identified a number of UV spectral features diagnostic of different iron-bearing minerals as well as a correlation in plagioclase between the depth of the $270 \mathrm{~nm}$ feature and the weight percent $\mathrm{Fe}_{2} \mathrm{O}_{3}$. The UV portion of ChemCam's spectral range is also expected to be used to investigate ozone in the Mars atmosphere. Secondly, ChemCam's high spectral resolution allows discrimination of spectrally narrow reflectance features that would not be identified by Mastcam filters. Clear examples are given in the features of coquimbite between 415 and $433 \mathrm{~nm}$ and for magnesiocopiapite and botryogen (a hydrated Mg/Fe sulfate) near $433 \mathrm{~nm}$ (Rossman 1975). Additionally, with its high spectral resolution, the team has demonstrated that Frauenhofer 
absorption lines are easily visible in the solar spectrum, suggesting additional possibilities, such as observing fluorescence within the absorption regions of the spectrum.

Much is still to be learned about the optimal use of ChemCam on Mars. Some of this necessarily deals with the small observation cross section of LIBS and how naturally heterogeneous samples will appear to the LIBS beam. Because of this, nearly all of the observations are expected to be multi-point rasters, such as those shown in Fig. 32. Additional work needs to be done to optimize spectral library training sets, and details on how to correct for variable observation distances still need to be completed.

Overall, ChemCam provides many capabilities never before deployed on Mars, including those of light element analyses, the sensitivity to characterize heavy alkali and alkaline earth elements (e.g., $\mathrm{Rb}, \mathrm{Sr}, \mathrm{Ba}$ ), ability to remotely remove dust and to study and remove weathering layers from rocks, and very fine spatial precision both in depth and laterally. The tactical roles it plays include reconnoitering nearby samples as well as assisting in selecting the precise locations for contact measurements and for obtaining samples for in-situ measurements. As such, it is anticipated that ChemCam will be used on various types of sols: reconnaissance, traverse sols, during approach, and also during contact and in-situ measurement sols. Estimates of the analyses to be conducted by ChemCam during the nominal mission range as high as 14,000 .

In a broader sense, the work described here and in related papers has significantly advanced the state of LIBS studies, leading the way in developing a compact and efficient LIBS system, in advancing chemometric techniques for analyses of geological samples (e.g., Clegg et al. 2009; Tucker et al. 2010; Dyar et al. 2011; Lasue et al. 2011; Anderson et al. 2011), and in providing both relative (e.g., Cousin et al. 2012) and absolute irradiance data of emission lines. Since the beginning of the ChemCam project, other teams have developed a strong interest in utilizing LIBS for planetary exploration.

Acknowledgements This work was supported by the NASA Mars Program in the US and CNES in France. Pre-selection development of LIBS for planetary science was supported in the US by grants from NASA's Mars Instrument Development Program (MIDP). The ChemCam team wishes to thank the additional people who supported the ChemCam program at various institutions, including Thomas Liimatainen who fabricated the calibration target body. Assistance at LANL by E. Michel, J. Salazar, R.D. Gurule, P. Archuleta, A. Mezzacappa, L.-M. Nortier, L. Salazar, D. Mietz, E. Fenimore, B. Dougherty, M. Fazio, and K. Saeger was greatly appreciated.

Open Access This article is distributed under the terms of the Creative Commons Attribution License which permits any use, distribution, and reproduction in any medium, provided the original author(s) and the source are credited.

\section{References}

R.B. Anderson, R.V. Morris, S.M. Clegg, J.F. Bell III, R.C. Wiens, S.D. Humphries, S.A. Mertzman, Icarus 215, 608 (2011)

W.C. Barkley, Mars rover-laser focusing and optimization. MS thesis, University of California, San Diego (2008)

J.F. Bell III et al., J. Geophys. Res. 108(E12), 8063 (2003). doi:10.1029/2003JE002070

D. Blake et al., Spa. Sci. Rev. (2012), this issue

J. Brückner, G. Dreibus, R. Reider, H. Wänke, J. Geophys. Res. 108(E12), 8094 (2003). doi:10.1029/ 2003JE002060

B.C. Clark, A.K. Baird, H.J. Rose Jr., P. Toulmin III, K. Keil, A.J. Castillo, W.C. Kelliher, C.D. Rowe, P.H. Evans, Science 194, 1283 (1976)

P.R. Christensen et al., J. Geophys. Res. 108(E12), 8064 (2003). doi:10.1029/2003JE002117 
S.M. Clegg, E. Sklute, M.D. Dyar, J.E. Barefield, R.C. Wiens, Spectrochim. Acta B 64, 79 (2009)

E.A. Cloutis, K.A. McCormack, J.F. Bell III, A.R. Hendrix, D.T. Bailey, M.A. Craig, S.A. Mertzman, M.S. Robinson, M.A. Riner, Icarus 197, 321 (2008)

P. Comon, in Higher-Order Statistics, ed. by J.L. Lacoume (Elsevier, Amsterdam, 1992), p. 29

A. Cousin, O. Forni, S. Maurice, J. Lasue, O. Gasnault, R. Wiens, the ChemCam team, Lunar Planet. Sci. LXII, 1973 (2011a)

A. Cousin, S. Maurice, O. Forni, R. Wiens, G. Berger, the ChemCam team, Lunar Planet. Sci. LXII, 1963 (2011b)

A. Cousin, O. Forni, O. Gasnault, C. Fabre, V. Sautter, R.C. Wiens, J. Mazoyer, Spectrochim. Acta B 66, 805 (2012)

D.A. Cremers, Appl. Spectrosc. 41, 572 (1987)

D.A. Cremers, L.J. Radziemski, in Laser-Induced Breakdown Spectroscopy (LIBS) Fundamentals and Applications, ed. by A.J.W. Miziolek, V. Palleschi, I. Schechter (Cambridge University Press, Cambridge, 2006), p. 1

L. Dudragne, P. Adam, J. Amouroux, Appl. Spectrosc. 52, 1321 (1998)

M.D. Dyar, J.M. Tucker, S. Humphries, S.M. Clegg, R.C. Wiens, M.D. Lane, Spectrochim. Acta B (2011). doi:10.1016/j.sab2010.11.016

T. Estlin, B. Bornstein, D. Gaines, D. Thompson, R. Castano, R.C. Anderson, C. de Granville, M. Burl, M. Judd, S. Chien, in Proceedings of the 10th International Symposium on Artificial Intelligence, Robotics and Automation in Space (iSAIRAS 2010), Sapporo, Japan (2010). http://robotics.estec.esa.int/ i-SAIRAS/isairas2010/PAPERS/073-2863-p.pdf

C. Fabre, S. Maurice, A. Cousin, R.C. Wiens, O. Forni, V. Sautter, D. Guilliame, Spectrochim. Acta B (2011). doi:10.1016/j.sab.2011.03.012

O. Forni, S. Clegg, R.C. Wiens, S. Maurice, O. Gasnault, Lunar Planet. Sci. XL, 1523 (2009)

R. Gellert et al., Spa. Sci. Rev. (2012), this issue

T.G. Graff, R.V. Morris, P.R. Christensen, Lunar Planet. Sci. XXXII, 1899 (2001)

S. Kaski, H. Häkkänen, J. Korppi-Tommola, Appl. Opt. 42, 6036 (2003)

C. Klein, Am. Mineral. 90, 1473 (2005). doi:10.2138/am.2005.1871

A.K. Knight, N.L. Scherbarth, D.A. Cremers, M.J. Ferris, Appl. Spectrosc. 54, 331 (2000)

G.A. Landis, the MER Athena Science Team, Lunar Planet. Sci. XXXVIII, 2423 (2007)

N.L. Lanza, S.M. Clegg, R.C. Wiens, R.E. McInroy, H.E. Newsom, M.D. Deans, Appl. Opt. 51, B74 (2012)

J. Lasue, R.C. Wiens, O. Forni, S.M. Clegg, S. Maurice, T. Stepinski, the ChemCam team, J. Anal. Bioanal. Chem. (2011). doi:10.1007/s00216-011-4747-3

V. Lazic, R. Barbini, F. Colao, R. Fantoni, A. Palucci, Spectrochim. Acta B 56, 807 (2001)

C.A. Lindensmith, W.T. Roberts, M. Meacham, M.N. Ott, F. LaRocca, W.J. Thomes, International Conference on Space Optics, October 4-8, Rhodes, Greece (2010). http://congrex.nl/icso/Papers/ Session\%207a/ICSO_paper_lindensmith_20100902.pdf

P. Mahaffy et al., Spa. Sci. Rev. (2012), this issue

S. Maurice et al., Spa. Sci. Rev. (2012), this issue

H.Y. McSween et al., J. Geophys. Res. 111, E02510 (2006). doi:10.1029/2005JE002477

R.V. Morris, T.G. Graff, T.D. Shelfer, J.F. Bell III, Lunar Planet. Sci. XXXII, 1912 (2001)

R.A. Multari, D.A. Cremers, J.M. Dupre, J.E. Gustafson, Appl. Spectrosc. 64, 750 (2010)

A.M. Ollila, J.G. Blank, R.C. Wiens, J. Lasue, H.E. Newsom, S.M. Clegg, A. Cousin, S. Maurice, Lunar Planet. Sci. XLII, 2395 (2011)

A.M. Ollila, J. Lasue, H.E. Newsom, R.A. Multari, R.C. Wiens, S.M. Clegg, Appl. Opt. 51, B130 (2012)

R. Perez, B.L. Barraclough, S.C. Bender, A. Cousin, A. Cros, L. DeFlores, N. LeRoch, S. Maurice, A. Paillet, L. Pares, Y. Parot, M. Saccoccio, R.C. Wiens, The ChemCam instrument for the 2011 Mars Science Laboratory Mission: System Requirements and Performance. 8th International Planetary Probe Workshop, June 6-10, Portsmouth, VA (2011). http://www.planetaryprobe.org/Portals/0/DynamicForms_Uploads/Perez_ChemCam-Paper.pdf

M. Pichavant, D.J. Kontak, J.V. Herrera, Contrib. Mineral. Petrol. 100, 300 (1988a)

M. Pichavant, D.J. Kontak, L. Briqueu, J.V. Hererra, A.H. Clark, Contrib. Mineral. Petrol. 100, 325 (1988b)

L.J. Radziemski, D.A. Cremers, M. Bostian, R.C. Chinni, C. Navarro-Northrup, Appl. Spectrosc. 61, 1141 (2007)

W.T. Roberts, C.A. Lindensmith, S. Bender, E.A. Miller, E. Motts, M. Ott, F. LaRocca, J. Thomes, International Conference on Space Optics, October 4-8, Rhodes, Greece (2010). http://congrex.nl/icso/Papers/Session\%207a/FCXNL-10A02-2018212-1-roberts_icso_fiber_paper.pdf

P. Rohwetter, K. Stelmaszczyk, L. Wöste, R. Ackermann, G. Méjean, E. Salmon, J. Kasparian, J. Yu, J.P. Wolf, Spectrochim. Acta B 60, 1025 (2005)

G.R. Rossman, Am. Mineral. 60, 698 (1975)

B. Sallé, D.A. Cremers, S. Maurice, R.C. Wiens, P. Fichet, Spectrochim. Acta B 60, 805 (2005) 
B. Sallé, P. Mauchien, S. Maurice, Spectrochim. Acta B (2007). doi:10.1016/j.sab.2007.07.001

J.W. Sammon Jr., IEEE Trans. Comput. C 18, 401 (1969)

S.K. Sharma, A.K. Misra, P.G. Lucey, R.C. Wiens, S.M. Clegg, Spectrochim. Acta A 68, 1036-1045 (2007)

J.-B. Sirven, B. Bousquet, L. Canioni, L. Sarger, Anal. Chem. 78, 1462 (2006)

S.W. Squyres, R.E. Arvidson, E.T. Baumgartner, J.F. Bell III, P.R. Christensen, S. Gorevan, K.E. Herkenhoff, G. Klingelhöfer, M.B. Madsen, R.V. Morris, R. Rieder, R.A. Romero, J. Geophys. Res. 108(E12), 8062 (2003). doi:10.1029/2003JE002121

R. Sullivan, R. Arvidson, J.F. Bell III, R. Gellert, M. Golombek, R. Greeley, K. Herkenhoff, J. Johnson, S. Thompson, P. Whelley, J. Wray, J. Geophys. Res. 113, E06S07 (2008). doi:10.1029/2008JE003101

J.M. Tucker, M.D. Dyar, M.W. Schaefer, S.M. Clegg, R.C. Wiens, Chem. Geol. 277, 137 (2010)

D.T. Vaniman, S.J. Chipera, D.L. Bish, Petrography, mineralogy, and chemistry of calcite-silica deposits at Exile Hill, Nevada, compared with local spring deposits. LA-13096-MS UC-802, Los Alamos National Laboratory (1995)

D.T. Vaniman, S. Clegg, N. Lanza, H. Newsom, R.C. Wiens, Lunar Planet. Sci. XL, 2296 (2009)

D.T. Vaniman, D. Dyar, R.C. Wiens, A. Ollila, N. Lanza, J. Lasue, M. Rhodes, S. Clegg, H.E. Newsom, Ceramic ChemCam calibration targets on Mars Science Laboratory (2012), this issue

R.C. Wiens, F.P. Seelos IV, M.J. Ferris, R.E. Arvidson, D.A. Cremers, J.D. Blacic, K. Deal, J. Geophys. Res. Planets (2002). doi:10.1029/2000JE001439

R.C. Wiens, L.E. Kirkland, C.P. McKay, D.A. Cremers, J. Thompson, S. Maurice, P.C. Pinet, Lunar Planet. Sci. XXXV, 1695 (2004)

R.C. Wiens, S.K. Sharma, J. Thompson, A. Misra, P.G. Lucey, Spectrochim. Acta A 61, 2324-2334 (2005)

J. Zipfel, C. Schröder, B. Jolliff, R. Gellert, K.E. Herkenhoff, R. Rieder, R. Anderson, J.F. Bell III, J. Brückner, J.A. Crisp, P.R. Christensen, B.C. Clark, P.A. de Souza Jr., G. Dreibus, C. D’Uston, T. Economou, S.P. Gorevan, B.C. Hahn, G. Klingelhöfer, T.J. McCoy, H.Y. McSween Jr., D.W. Ming, R.V. Morris, D.S. Rodionov, S.W. Squyres, H. Wänke, S.P. Wright, M.B. Wyatt, A.S. Yen, Meteorit. Planet. Sci. 46, 1-20 (2011) 